\title{
Agent-Based Macroeconomics
}

\author{
H. Dawid D. Delli Gatti
}




\title{
Agent-Based Macroeconomics
}

\author{
Herbert Dawid* $\quad$ Domenico Delli Gatti ${ }^{\dagger}$
}

January 2018

This paper has been prepared as a chapter in the Handbook of Computational Economics, Volume IV, edited by Cars Hommes and Blake LeBaron.

\begin{abstract}
This chapter surveys work dedicated to macroeconomic analysis using an agentbased modeling approach. After a short review of the origins and general characteristics of this approach a systemic comparison of the structure and modeling assumptions of a set of important (families of) agent-based macroeconomic models is provided. The comparison highlights substantial similarities between the different models, thereby identifying what could be considered an emerging common core of macroeconomic agent-based modeling. In the second part of the chapter agent-based macroeconomic research in different domains of economic policy is reviewed.
\end{abstract}

Keywords: Agent-based Macroeconomics, Aggregation, Heterogeneity, Behavioral Rules, Business Fluctuations, Economic Policy

JEL Classification: C63, E17, E32, E70,

\section{Introduction}

Starting from the early years of the availability of digital computers, the analysis of macroeconomic phenomena through the simulation of appropriate micro-founded models of the economy has been seen as a promising approach for Economic research. In an article in the American Economic Review in 1959 Herbert Simon argued that

"The very complexity that has made a theory of the decision-making process essential has made its construction exceedingly difficult. Most approaches have been piecemeal-now focused on the criteria of choice, now on conflict of interest, now on the formation of expectations. It seemed almost utopian to suppose that we could put together a model of adaptive man that would compare in completeness with the simple model of classical economic man. The sketchiness and incompleteness of the newer proposals has been urged as a compelling reason

*Department of Business Administration and Economics and Center for Mathematical Economics, Bielefeld University, P.O. Box 100131, 33501 Bielefeld, Germany. Email: hdawid@wiwi.uni-bielefeld.de.

${ }^{\dagger}$ Complexity Lab in Economics (CLE), Department of Economics and Finance, Università Cattolica del Sacro Cuore. Email: domenico.delligatti@unicatt.it

${ }^{\ddagger}$ CESifo Group Munich, Germany 
for clinging to the older theories, however inadequate they are admitted to be. The modern digital computer has changed the situation radically. It provides us with a tool of researchfor formulating and testing theories-whose power is commensurate with the complexity of the phenomena we seek to understand. [...] As economics finds it more and more necessary to understand and explain disequilibrium as well as equilibrium, it will find an increasing use for this new tool and for communication with its sister sciences of psychology and sociology." [Simon (1959), p.280].

This quote, which calls for an encompassing macroeconomic modelling approach building on the interaction of (heterogeneous) agents whose expectation formation and decision making processes are based on empirical and psychological insights, might be seen as the first formulation of a research agenda, which is now referred to as 'Agent-based Macroeconomics'. Following this agenda in the 1970s micro founded simulation models of the Swedish economy (the MOSES model, see Eliason $(1977,1984)$ ) and the U.S. economy (the 'Transactions Model', see Bergman (1974), Bennett and Bergmann (1986)) have been developed as a tool for the analysis of certain economic policy measures. Although calibrated for specific countries, the structure of these models was rather general and as such they can be seen as very early agent-based macroeconomic models.

At the same time, starting in the 1970s the attention of the mainstream of macroeconomic research has shifted towards (dynamic) equilibrium models as a framework for macroeconomic studies and policy analyses. At least in their original form these models are built on assumptions of representative agents, rational expectations and equilibrium based on inter-temporally optimal behavior of all agents. The clear conceptual basis as well as the relatively parsimonious structure of these models and the fact that they address the Lucas critique has strongly contributed to their appeal and has resulted in a large body of work dedicated to this approach. In particular, these models have become the workhorse for macroeconomic policy analysis.

Nevertheless, already early in this development different authors have pointed out numerous problematic aspects associated with using such models, in particular Dynamic Stochastic General Equilibrium (DSGE) models, for economic analysis and policy studies. Kirman (1992) nicely summarizes results showing that in general aggregate behavior of a heterogeneous set of (optimizing) agents cannot be interpreted as the optimal decision of a representative agent and that, even in cases where it can, the sign of effects of policy changes on the utility of that representative agent might be different from the sign of the induced utility changes of all agents in the underlying population, which makes the interpretation of welfare analysis in representative agent models problematic. Furthermore, an extensive stream of literature has shown that under reasonable informational assumptions no adjustment processes ensuring general convergence to equilibrium can be constructed (see Kirman (2016)). Hence, the assumption of coordination of all agents in an equilibrium is very strong, even if a unique equilibrium exists, which in many classes of models is not guaranteed. As argued e.g. in Howitt (2012) this assumption also avoids addressing coordination issues in the economy, which are essential for understanding the phenomena like the emergence of crises and also the impact of policies. Similarly, the assumption that all agents have rational expectations about the future dynamics of the economy has been criticized as being rather unrealistic and indeed there is little experimental or empirical evidence suggesting that the evolution of expectations of agents is consistent with the rational expectations assumption (see e.g. Carroll (2003) or Hommes et al. (2005)).

From a more technical perspective, studies in the the DSGE literature typically rely on 
local approximations of the model dynamics around a steady state (e.g. log-linearisation) and thereby do not capture the full global dynamics of the underlying model. This makes it problematic to properly capture global phenomena like regime changes or large fluctuations in such a framework. Business cycles and fluctuations are driven by shocks to fundamentals or expectations, whose structure is calibrated in a way to match empirical targets. Hence, the mechanisms actually generating these fluctuations are outside the scope of the model and therefore the model can only be used to study propagation of shocks, but is silent about which mechanisms generate such phenomena and which measures might reduce the risk of the emergence of cycles and downturns in the first place.

In the aftermath of the the crises developing after 2007 policy makers as well as Economists have acknowledged that several of the properties mentioned above substantially reduce the ability of standard DSGE models to inform policy makers about suitable responses to the unfolding economic downturn. New generations of DSGE-type models have been developed addressing several of these issues, in particular introducing more heterogeneity [see chapter by Ragot in this handbook], heterogeneous non-rational expectations [see chapter by Branch and McGough in this handbook] or the feedback between real and financial dynamics (e.g. Benes et al. (2014)), however also in each of these extensions several of the points discussed above, which seem intrinsic associated with this approach, still apply. ${ }^{1}$

Related to these new developments also a stream of literature has emerged, which, although relying on the backbone of a standard DSGE-type model, in certain parts of the model incorporates explicit micro-level representations of the (local) interaction of agents and relies on (agent-based) simulation of the emerging dynamics. Although these contributions (e.g. Anufriev et al. (2013), Arifovic et al. (2013), Assenza and Gatti (2013, 2017), Lengnick and Wohltmann (2016)) can be considered to be part of the agent-based macroeconomic literature, they are more hybrid in nature and are treated in the Chapter [Branch and McGough] of this handbook, rather than in this chapter. ${ }^{2}$

The agent-based approach to macroeconomic modeling, which has started to attract increasing attention from the early 2000s onwards, is similar in spirit to Simon's quote above and hence differs in several ways from mainstream dynamic equilibrium models. In agent-based macroeconomic models different types of heterogeneous agents endowed with behavioral and expectational rules interact through explicitly represented market protocols and meso- as well as macroeconomic variables are determined by actual aggregation of the output in this population of agents. They are mainly driven by the desire to provide empirically appealing representations of individual behavior and interaction patterns on the micro level and at the same time to validate the models by comparing the characteristics of their aggregate level output with empirical data. The global dynamics of the models are studied relying on (batches of) simulation runs and typically no ex-ante assumptions about the coordination of individual behaviour is made.

Already early contributions to this stream of literature have shown that these types of models can endogenously generate fluctuations resembling actual business cycles without relying on external shocks (e.g. Dosi et al. (2006)) and have highlighted, before the outbreak of the crises of 2007, mechanisms by which contagion (e.g. through credit networks) and feed-

\footnotetext{
${ }^{1}$ More extensive critical discussions of different aspects of the DSGE approach to macroeconomic modeling can be found in Colander et al. (2008), Fagiolo and Roventini (2011, 2017) or Romer (2016), where in particular Fagiolo and Roventini (2017) also consider different recent extensions to the DSGE literature

${ }^{2}$ Early predecessors in a similar spirit are e.g. Arifovic $(1995,1996)$, in which agent-based computational learning models have been incorporated into standard macroeconomic settings.
} 
back between the real and financial side of the economy can induce instability and sudden downturns (see Battiston et al. (2007)). These properties together with the ability to incorporate a wide range of behavioral assumptions and to represent institutional characteristics, which might be relevant for the analysis of actual policy proposals, have fostered interest of policy makers for agent-based macroeconomic modeling ${ }^{3}$ and resulted in a vast increase in research in this area in general and in agent-based policy analysis in particular. As has to be expected from a new emerging paradigm, the evolution of the field has progressed in several weakly coordinated streams and in light of the large body of work that has been produced so far, a systematic review of the progress that has been made seems to be in order. This chapter is an attempt to provide such a review.

\subsection{Complexity and Macroeconomics}

The notion of complexity is general enough to encompass a broad class of phenomena and models in nature and society. We interpret complexity as an attribute of a system. In particular, following the approach pioneered at the Santa Fe Institute by an interdisciplinary group of prominent scientists, Complex adaptive systems (CAS) are systems consisting of a large number of "coupled elements the properties of which are modifiable as a result of environmental interactions.[...] In general complex adaptive systems are highly non-linear and are organized on many spatial and temporal scales" (cited from Cowan and Feldmann in Fontana (2010)[p.173]).

Macroeconomic dynamics are characterized by the interaction of a large number of heterogeneous individuals who take a plethora of decisions of different kinds to produce and exchange a large variety of goods as well as information. These transactions are governed by institutional rules which might vary significantly between different regions, industries, time periods and other contexts. Based on this, economic systems must certainly be seen as very complex adaptive systems. This makes it extremely challenging to develop appropriate models for studying economic systems and to derive any insights of general validity about the (future) dynamics of key economic variables or the effect of certain economic policy measures.

In order to study CAS a natural tool is an Agent Based Model (ABM), i.e., a model in which a multitude of of (heterogeneous) elements or objects interact with each other and the environment. The single most important feature of an ABM is the autonomy of the elements, i.e. the absence of a centralized ("top down") coordinating or controlling mechanism. ABM are, by construction, computationally intensive. The output of the model typically cannot be determined analytically but must be computed and consists of simulated time series. A key feature of CAS is that it often gives rise to emerging properties, i.e. stable, orderly aggregate structures resulting from the interaction of the agents' behaviour. A phenomenon is emergent whenever the whole achieves properties which its element, if taken in isolation, do not have.

\subsection{The Agent Based Approach to Macroeconomic Modelling}

Agent based Computational Economics (ACE) is the application of AB modeling to economics or: "The computational study of economic processes modelled as dynamic systems

\footnotetext{
${ }^{3}$ Clear indications of the potential that central banks see in agent-based macroeconomics can be found in Trichet (2010) or Haldane (2016).
} 
of interacting agents." (Tesfatsion (2006)). Surveys of the ample literature on ACE work in different areas of Economics are provided in the second volume of the Handbook of Computational Economics (Tesfatsion and Judd (2006)). It is worthwhile noting that in this Handbook no separate chapter on agent-based macroeconomics was included, which is a signal of the limited work in this area that has been completed before 2006 .

A defining feature of macroeconomic $A B M s(M A B M s)$ is that although concerned with the dynamics of aggregate economic variables, such as GDP, consumption etc., they explicitly capture the micro-level interaction of different types of heterogeneous economic agents and allow to compute the aggregate variables "from the bottom up", i.e. summing individual quantities across agents. The bottom-up approach to macroeconomics consists therefore in deducing the macroscopic patterns and phenomena in terms of a multitude of elementary microscopic objects (micro-economic variables) interacting according to certain rules and protocols.

Developing and using a MABM typically requires a number of steps:

- Model Design and Theory:

- determine the type of agents included to be in the model (households, firms, banks,...).

- for each agent of each type define the set of decisions to be taken, the set of internal states (e.g. wealth, skills, savings,..), structure of each decision rule (inputs, how is decision made), the potential information exchange with other agents and the potential dynamic adjustment of internal states and decision rules; decide on the theoretical, empirical or experimental foundations on which these choices are based.

- define interaction protocols for all potential interactions.

- Codification: translate the rules into computer code, do proper testing of the code (e.g. unit testing) to ensure proper implementation of the model.

- Parameter Choice and Validation: estimate respectively calibrate the parameters; run simulations; analyze the emerging properties of the simulated data, both at the crosssectional level (e.g. firms' size distribution) and at the macroeconomic level (GDP growth and fluctuations, inflation/unemployment trade off); compare these properties with real world "stylized facts".

- Model Analysis: study the effects of changes in key model parameters (e.g. policy parameters) based on proper statistical analysis of the output of batch runs across different parameter settings; use micro-level simulation data to highlight the mechanisms responsible for the observed findings and to foster economic intuition for the findings.

Several properties are common to MABMs and have been encountered in many agentbased models in Economics, among them several of the models reviewed in this chapter. First and foremost, in MABMs GDP tends to self-organize towards a growth path with endogenously generated fluctuations, such that business cycles are driven by the mechanics of the model rather than by properties of exogenous shocks. Furthermore, these model typically generate persistent heterogeneity of agents, giving rise to stable population distributions of firm size, productivity, profitability, growth rate or household income and thereby can reproduce also empirical patterns with respect to distributions of such variables. In particular, 
distributions with fat tails, which are observed for many real world variables, have been reproduced in many instances by MABMs. Being able to jointly reproduce empirical stylized facts with respect to time series properties and distributional properties at different levels of aggregation is certainly a very appealing feature of MABMs, which is hard to obtain in the framework of alternative macroeconomic modeling approaches.

Many MABMs are characterized by externalities and non-linearities (due to interaction), which generate dynamic processes with positive feedbacks. Due to the presence of such feedbacks path dependencies might arise such that initial conditions or random events in the transient phase can have decisive impact on the long run dynamics. These properties of MABMs are also the basis for endogenously generating extreme events, like crashes and economic crises as well as fast transitions between different quasi-stable regimes. MABMs capture the actual dynamic mechanisms generating such potential fast economic transitions and therefore are natural tools to study how to prevent or mollify economic crises, e.g. through appropriate institutional designs or policy measures. Generally speaking, the fact in the agent-based models allow for global analysis of macroeconomic dynamics in frameworks which endogenously generate dynamic and cross-sectoral patterns, which closely resemble empirical data, arguably is a main reason for the appeal of this approach.

\subsection{Behaviour, Expectations and Interaction Protocols}

Other than dynamic equilibrium models, in which individual behaviour is typically determined by the optimal solution of some (dynamic) optimization problem an agent with rational expectations faces, in agent-based macroeconomic models it is not assumed that the economy is in equilibrium and that individuals have rational expectations. Hence, the agents in the model, similarly to real-world decision makers, are "necessarily limited to locally constructive actions, that is, to actions constrained by their interaction networks, information, beliefs, and physical states." (Sinitskaya and Tesfatsion (2015),[p.152]). The design of behavioural rules determining such locally constructive actions is a crucial aspect of developing an agent-based macroeconomic model. The lack of an accepted precise common conceptional or axiomatic basis for the modeling of bounded rational behaviour has raised concerns about the "wilderness of bounded rationality" (Sims (1980)), however agent-based modelers have become increasingly aware of this issue providing different foundations for their approaches to model individual behavior.

Generally speaking, in many MABMs the design of the behavioral rules builds on the extensive psychological and empirical literature showing the prevalence of relatively simple heuristics respectively rules of thumb for making decisions, including economic decisions in complex environments (see e.g. Gigerenzer and Gaissmaier (2011), Artinger and Gigerenzer (2016)). Such rules might be derived from optimization within the framework of a simplified internal model of the surrounding environment, or might evolve over time based on adjustment dynamics that take into account which types of rules generate desirable results for the decision maker. In a number of agent-based models the chosen behavioral rules are strongly motivated by experimental ${ }^{4}$ or empirical observations of how actual decision makers behave in certain types of decision problems ${ }^{5}$. As will become in clear in our survey below, the

\footnotetext{
${ }^{4}$ See Hommes (2013) or Assenza et al. (2015b) for a discussion of the use of laboratory experiments as the foundation for the formulation of heuristic behavioural rules.

${ }^{5}$ With respect to firm decisions the 'Management Science Approach', see Dawid and Harting (2012), has been put forward as a way to incorporate decision rules into agent-based models, that resemble heuristics
} 
literature shows substantial heterogeneity with respect to the approach that underlies the design of the behavioural rules. Similar statements apply to the expectation formation of agents. The absence of the assumption of rational expectations gives typically rise to models with evolving heterogeneous expectations and also in this domain different approaches have been followed.

Given the heterogeneity in the way decision making and expectation formation are modeled it would be desirable to have a clear understanding of how robust results obtained in the framework of a certain model are with respect to the use of alternative plausible behavioral rules. A step in this direction is taken by Sinitskaya and Tesfatsion (2015), who compare in a simple macroeconomic framework how key outcomes of the model compare across settings with different types of decision rules, however in particular in large macroeconomic models such types of robustness tests are not feasible and the chosen design of the behavioural rules might therefore be an important determinant of the model output.

In most macroeconomic agent-based models the interaction of the different agents in markets or other interaction structures are governed by explicit protocols that represent the institutional design of the considered economic system. This allows capturing details of the institutional setting and also allows representing in a natural way potential rationing of both market sides as well as the occurrence of frictions in a market. The degree of detail with which the interactions structures in different markets are described of course varies substantially across the agent-based macroeconomic models that have been developed and is strongly influenced by the main focus of the model.

\subsection{Outline of the Chapter}

In this chapter we discuss the main developments in Agent-based Macroeconomics during the last decade. The treatment is essentially split into two parts. In the first part, consisting of Sections 2 and 3, we focus on the design of macroeconomic agent-based models. In particular, in Section 2 we address in some detail several main challenges of macroeconomic agent-based modeling, in particular the design of the behavioral rules of different types of agents for several of the most crucial decisions to be taken. We illustrate how these challenges were treated in eight macroeconomic agent-based models, that have been well perceived in the literature. In Section 3, we provide more of a bird's-eye view on these model by summarizing the detailed discussion of Section 2 and providing a systematic comparison of these eight MABMs along a larger number of modeling dimensions. Section 3 also contains a discussion of the way these models have been linked to empirical data. The discussion in Section 3 is based on Tables A1 and A2, provided in Appendix A, in which a short summary of the main features of all eight models is given. Overall, we hope that the Sections 2 and 3 of the chapter do not only provide a survey of the literature, but are also helpful in identifying what could be considered a common core of macroeconomic agent-based modeling The second part of the chapter, essentially Section 4, provides an overview over macroeconomic policy analyses that have been carried out using agent-based models. Although the eight models discussed in Sections 2 and 3 are the basis for a considerable fraction of this policy oriented work, numerous studies reviewed in Section 4 do not fall into this category. This highlights the breadth of work in agent-based macroeconomics during the last years and the fact that

developed in the literature on managerial decision making. The underlying rationale of this approach is that actual decision making of managers is likely to be guided by these heuristics which are put forward in textbooks and taught in Business Schools. 
a chapter like this, due to space constraints, cannot properly capture the full status of the literature. The chapter concludes with some remarks about challenges for the future development of this line of research and about areas in which in our opinion the potential for agent-based analysis is particularly high.

\section{Design of Agent-based Macroeconomic Models}

\subsection{Families of MABMs}

Macroeconomic Agent-Based Models (MABMs) can be classified according to different criteria. First of all, we can distinguish between large, medium sized and small MABMs.

Medium sized and large MABMs feature at least three agents' types -households, firms and banks - interacting at least on five markets: consumption goods (C-goods hereafter), capital or investment goods (K-goods), labor, credit, deposits. Small MABMs generally feature just two types of agents - households and firms - interacting on two markets: Cgoods and labor.

Some MABMs are able to replicate growth - i.e. a long run exponential trend around which actual GDP irregularly fluctuates - some other focus only on the short run, i.e., they can replicate only business fluctuations.

In this section and the next, we will focus on medium-sized MABMs, grouping them into seven families:

1. the framework developed by Ashraf, Gershman and Howitt (AGH hereafter $)^{6}$;

2. the family of models proposed by Delli Gatti, Gallegati and co-authors in Ancona and Milan exploiting the notion of Complex Adaptive Trivial Systems (CATS) ${ }^{7}$;

\footnotetext{
${ }^{6}$ In the following we will refer mainly to Ashraf et al. (2016) and Ashraf et al. (2017). For an extension and application to monetary and macro-prudential policy, see Popoyan et al. (2017)

${ }^{7}$ Delli Gatti et al. (2005) is the most significant early example of a CATS model, populated by myopic optimizing firms, which use only capital to produce goods. Russo et al. (2007) develop an early model along similar lines, with an application to fiscal policy. Some reflections on building macro ABMs stimulated by these early experiences can be found in Gaffeo et al. (2007). Gaffeo et al. (2015) put forward a model with learning and institutions. The single most important CATS framework, which is at the core of a wave of subsequent models, is described in chapter 3 of the book "Macroeconomics from the Bottom Up" (Delli Gatti et al. (2011)). We will refer to this framework as CATS/MBU. CATS/MBU features households, firms and banks. Firms use only labor to produce consumption goods. The properties of this model in a strippeddown version (without banks) have been analyzed in depth in Gualdi et al. (2015). A CATS/MBU set up has been used by Delli Gatti and Desiderio (2015) to explore the effects of monetary policy (hereafter CATS/DD). Klimek et al. (2015) use a variant of CATS/MBU to analyze bank resolution policies. Assenza et al. (2015a) have extended the model introducing capital goods (hereafter CATS/ADG). There are quite a few networked MABMS of the CATS family. A first wave of network based financial accelerator models consists of Delli Gatti et al. (2006), Delli Gatti et al. (2009), further developed in Delli Gatti et al. (2010). A new wave of networked MABMS exploits the dynamic trade off theory: Riccetti et al. (2013), Bargigli et al. (2014) and Riccetti et al. (2016b). Using a similar set up, Catullo et al. (2015) develop early warning indicators of an incoming financial crisis. A medium-to large model (so called "modellone") is an extension of the previous framework: Riccetti et al. (2015). For applications of this model to different topics, see Riccetti et al. (2013), Riccetti et al. (2016a), Russo et al. (2016), Riccetti et al. (2018). Caiani et al. (2016a) develop a medium-to large model with emphasis on stock-flow consistency (so called "modellaccio"). For an application of this model to inequality and growth, see Caiani et al. (2016b).
} 
3. the framework developed by Dawid and co-authors in Bielefeld as an offspring of the EURACE project $^{8}$, known as Eurace@Unibi (EUBI) ${ }^{9}$;

4. the EURACE framework maintained by Cincotti and co-authors in Genoa (EUGE) ${ }^{10}$,

5. the Java Agent based MacroEconomic Laboratory developed by Salle and Seppecher $(\text { JAMEL })^{11}$;

6. the family of models developed by Dosi, Fagiolo, Roventini and co-authors in Pisa, known as the "Keynes meeting Schumpeter" framework $(\mathrm{KS})^{12}$;

7. the LAGOM model developed by Jager and co-authors. ${ }^{13}$.

We will also present the relatively simple small model developed by Lengnick (LEN) for

${ }^{8}$ The EURACE project was funded by the European Commission 2006-2009 under the 6th Framework programme, and was carried out by a consortium of 7 universities (located in France, Germany, Italy, UK and Turkey), coordinated by Silvano Cincotti (University of Genoa). The agenda of the project was to develop an agent-based simulation platform that is suitable for (macro)economic analysis and the evaluation of the effect of different types of economic policy measures. See Holcombe et al. (2013), Deissenberg et al. (2008) and Cincotti et al. (2012a) for descriptions of the agenda of the project and the version of the model as developed during the EURACE project.

${ }^{9}$ For an extensive presentation of the model see Dawid et al. (2018c). A concise discussion can be found in Dawid et al. (2018a). For an application to firm dynamics, see Dawid and Harting (2012). Fiscal policies are analysed in Harting (2015) and Dawid et al. (2018b). Two papers on financial and macro-prudential issues: van der Hoog and Dawid (2017), van der Hoog (2018). The nexus of skill dynamics, innovation and growth in multi-regional settings is explored in Dawid et al. $(2008,2013,2014)$. Labor market integration policies are analyzed in Dawid et al. (2012). In Dawid and Gemkow (2014) social networks are integrated into the model and their role for the emergence of income inequality is studied.

${ }^{10}$ After the end of the EURACE project, the EURACE model has been maintained at the university of Genoa and been adapted both in size and scope to different research questions. The group we will refer to as EUGE is currently running different specifications of the framework. For applications to the interaction between the banking system and the macroeconomy and the analysis of the effects of financial regulation, see Cincotti et al. (2010), Teglio et al. (2010), Teglio et al. (2012), Cincotti et al. (2012b), Raberto et al. (2012), Raberto et al. (2017). For the analysis of monetary policy, see Raberto et al. (2008). For the analysis of the effects of fiscal policy and sovereign debt, see Raberto et al. (2014), Teglio et al. (2018). For applications to the housing and mortgage markets, see Erlingsson et al. (2013), Erlingsson et al. (2014), Teglio et al. (2014), Ozel et al. (2016). For an application to the issues pertaining to energy, see Ponta et al. (2018). Finally, a multi-country version is analyzed in Petrovic et al. (2017). In this section we will refer to the most general features of the model, which we retrieve mainly from Cincotti et al. (2012a).

${ }^{11}$ The building blocks of the JAMEL model are described in Seppecher (2012). Seppecher and Salle (2015) explore the emergent properties of the model, namely the alternating macroeconomic regimes of boom and bust. A model with emphasis on stock-flow consistency is presented in Seppecher et al. (2018). The role of expectations in macro ABMs is thoroughly analyzed in Salle et al. (2013) and Salle (2015).

${ }^{12}$ Early examples of ABMs which will eventually develop into the KS framework are Dosi et al. (2006) and Dosi et al. (2008). In the following we will discuss mainly the model in Dosi et al. (2010), which has been extended to introduce banks and macro-financial interactions (and to be used for fiscal, monetary and prudential policy exercises) in Dosi et al. (2013),Dosi et al. (2015),Dosi et al. (2017a). The model has been used to analyze labor market issues and the effects of structural reforms in Napoletano et al. (2012), Dosi et al. (2017d), Dosi et al. (2018), Dosi et al. (2017b). For an application to the analysis of the effects of climate change, see Lamperti et al. (2017). For a general overview of this KS literature see Dosi et al. (2017a)

${ }^{13}$ LAGOM is not an acronym (as in the case of the other families of MABMs) but a Swedish word which means equilibrium and harmony "perhaps akin to the chinese Tao." (Haas and Jaeger (2005), p. 2). In the following we will discuss mainly the model in Wolf et al. (2013), Mandel et al. (2010) 
comparison. ${ }^{14}$ Key references for these models are given in Tables A1 and A2.

By selecting these eight families of models, on the one hand, we tried to pick those that seem to have the strongest impact on the literature and have been used as the basis for interesting economic analyses and policy experiments, and, on the other hand, also present some variety to show the range of approaches that have been developed to deal with the challenges of agent-based macroeconomic modeling Clearly, any such selection is highly subjective and, as will also become clear in the discussion of agent-based policy analyses in Section 4, the selection made here misses a substantial number of important contributions to this area. Nevertheless, we believe that presenting such a survey is not only useful for newcomers to the field, but also helps to provide transparency about the status of the field of agent-based macroeconomics. Due to the rather complex structure of many models in this field, which often makes a full model descriptions rather lengthy, such transparency is not easy to obtain.

The interested reader who ventures for the first time into this literature may feel the excitement of exploring a new world and, at the same time, the disorientation and discouragement of getting lost in the wilderness. At first sight, in fact, these models look very different from one another so that it's extremely difficult for the beginner to "see the forest" above and beyond a wide variety of trees. In our opinion, however, there are common denominators, both in the basic architecture of the models and in the underlying theory of the way in which agents form behavioral rules and interact on markets.

\section{$2.2 \quad$ A map of this section}

Let us set the stage by considering the architecture of a MABM. The economy is populated, at a minimum, by households and firms (as in LEN). Medium sized MABMs, are populated also by banks.

Households supply labor and demand C-goods. In most MABMs households are "surplus units", i.e., net savers. Savings are used to accumulate financial wealth.

The corporate sector consists, at a minimum, of producers of C-goods (C-firms). Most MABMs, however, are now incorporating also producers of K-goods (K-firms). C-firms demand labor and K-goods in order to produce and sell C-goods to households. K-firms supply K-goods to C-firms.

In most MABMs firms are "deficit units", i.e., firms' internal funds may not be sufficient to finance costs. Therefore they resort to external finance to fill the financing gap. In most MABMs external finance coincides with bank loans. Banks receive deposits from households and extend loans to firms.

In small MABMs, such as LEN, there are markets for C-goods and labor. In medium sized MABMs, there are typically markets for C-goods, K-goods, labor, credit and deposits. ${ }^{15}$ Given this architecture, we can allocate agents in markets according to the following grid.

Each column of the table represents a group of agents, each row a market. For instance $\mathrm{H} / \mathrm{C} / \mathrm{d}$ represents Households acting on the market for $\mathbf{C}$-goods on the side of demand.

Instead of reviewing the models one after the other, in each of the following subsections we will discuss the characterizations that the proponents of different MABMs adopt to describe

\footnotetext{
${ }^{14}$ In the following we will discuss mainly the model in Lengnick (2013). See also Lengnick and Wohltmann (2016) and Lengnick and Wohltmann (2011).

${ }^{15}$ It should be mentioned that for several of the MABMs there exist also variants including additional markets, e.g. for housing and electricity, see Lamperti et al. (2017), Ozel et al. (2016).
} 


\begin{tabular}{|l|l|l|l|}
\hline & Households & Firms & Banks \\
\hline C-goods & $\mathrm{H} / \mathrm{C} / \mathrm{d}$ & $\mathrm{F} / \mathrm{C} / \mathrm{s}$ & \\
K-goods & & $\mathrm{F} / \mathrm{K} / \mathrm{d}, \mathrm{s}$ & \\
Labour & $\mathrm{H} / \mathrm{N} / \mathrm{s}$ & $\mathrm{F} / \mathrm{N} / \mathrm{d}$ & \\
Credit & & $\mathrm{F} / \mathrm{L} / \mathrm{d}$ & $\mathrm{B} / \mathrm{L} / \mathrm{s}$ \\
Assets & $\mathrm{H} / \mathrm{A} / \mathrm{d}$ & $\mathrm{F} / \mathrm{A} / \mathrm{s}$ & \\
\hline
\end{tabular}

Table 1: $H$ stands for households; $F$ denotes firms; $B$ stands for banks; $N$ denotes the labor market; $L$ denotes the market for loans; $A$ denotes the market for assets.

the behavioral rules that each type of agent (on the columns) follows in each of the different markets the agent is active (on the rows). We will also devote some space to the description of the interaction of buyers and sellers on markets (market protocols) which Tesfatsion labels "procurement process". 16

We aim at bringing to the fore the similarities among different MABMs. As mentioned above, assumptions and modeling choices come from a variety of sources, first and foremost from the empirical and experimental evidence. It is worth noting,moreover, that these assumptions have a varying degree of kinship with the current macroeconomic literature. MABMs are not developed in a vacuum, the shapes of their building blocks come also from the theoretical debate in macroeconomics. For this reason, at the beginning of each subsection, we will succinctly present the microeconomic backbone of a standard New Keynesian DSGE (NK-DSGE) model (the standard model hereafter) pertaining to that class of agents, ${ }^{17}$ then present the behavioral rules and market protocols of MABMs concerning the same class. In this way we can discuss similarities and differences (i) between the standard model and the MABMs and (ii) among MABMs. In order to make the comparison easier, we will adopt our own notation, which will be uniform across different MABMs. We will also slightly simplify the analytical apparatus of a specific MABM under review to make the modeling choices starker in the eyes of the reader. Notice finally that we will consider each MABM as the result of a collective effort (with the exception of LEN). Hence we will conjugate a verb describing the action of the group behind the label of each MABM in the third person plural. To foster the readability we provide in Appendix B a list of symbols with their meaning that are used in this section.

In our presentation of MABMs, due to space limitations we will not discuss three relevant features.

The first concerns the sequence of events, which may differ from one model to the other. By construction, MABMs are recursive sequential models. Agents decide on the desired level of their choice variables (planned level) following behavioral rules and then enter markets one after the other in order to implement those decisions by suitable transactions. Search of trading opportunities and matching of supply and demand occur in fully decentralized

\footnotetext{
${ }^{16}$ For a discussion of this notion, see Tesfatsion (2006).

${ }^{17}$ The NK-DSGE literature is immense. The prototypical small standard model is the microfounded NKDSGE "three equation model", which is routinely taught in core macroeconomics courses at the graduate level. Among textbooks, the obvious reference is Gali (2008). This model features only two classes households and firms (epitomized by a representative agent per class) - and two markets, goods and labor. The introduction of the banking system is due to Bernanke et al. (1999) (BGG). Nowadays, BGG is the standard medium-sized NK-DSGE model with financial frictions.
} 
markets, i.e., in the absence of a top-down mechanism to enforce equilibrium. Therefore, transactions typically occur at prices which do not clear the market. This may cause a disruption of plans, which must be revised accordingly.

Consider, for instance, a C-firm. Once the quantity to be produced has been established, the firm determines desired employment. If desired employment is greater than the current workforce, the firm tries to hire new workers by posting vacancies. She may not be able to fill the vacancies, however, because not enough workers will visit the firm or accept the position. ${ }^{18}$ In this case, the firm has to downsize her production plans. Generally, due to unexploited trading opportunities, three constraints may limit the implementation of decisions, e.g. firms may be unable to (i) find enough external funds to fill the financing gap and/or (ii) hire enough workers and/or (iii) acquire enough capital to implement the desired level of production.

The second feature concerns time discretization. By construction, in MABMs time is discrete. MABMs can differ, however, as far as the minimal time unit is considered (a day, a week, a month, a quarter). Moreover, transactions can occur at different time scales. For instance, in LEN C-goods are traded every day but labor services are traded on a monthly base. In the following, for simplicity we will not be specific on the time unit which will be referred to with the generic term "a period".

The third feature concerns the characterization of interaction. A few MABMs are networked, i.e. they have an explicit network structure: agents are linked by means of trading relationships which take the form of persistent partnerships. In LEN, for instance, each household trades with a finite set of firms. ${ }^{19}$ Most of the MABMs we will consider below, however, do not assume a fixed network of trading relationships. Partners in a trade today may not trade again tomorrow. In a sense, trading relationships de facto connect people in a network which is systematically reshuffled every period. ${ }^{20}$

\subsection{Households}

In the following, we will consider a population of $H$ households. Variables pertaining to the h-th household will be denoted with the suffix $h$. Households may be active or inactive on the labor market. If active, they supply labor (in most MABMs, labor supply is exogenous). In some MABMs, households searching the labour market have a reservation wage, which may be constant or decreasing with the length of the unemployment spell. If employed, the household earns a wage. In some MABMs, if unemployed the household receives an unemployment subsidy, which amounts to a fraction of the wage of employed households.

Households are also firm owners. Firm ownership may be limited to a fraction of inactive households or spread somehow also to active households. As a firm owner, the household receives dividends. Current income is the sum of the wage bill and dividends. Households purchase C-goods. Generally, households are surplus units, i.e., they do not get into debt. Unspent income is saved and generates financial wealth. In most MABMs, financial wealth

\footnotetext{
${ }^{18}$ In most MABMs the labour market is riddled with frictions: each unemployed worker visits only a limited number of firms and/or the posted vacancies are advertised only to a certain number of unemployed workers. Hence, after a round of transactions on the market, there will still be unfilled vacancies as well as unemployed workers.

${ }^{19}$ This is also the case, for instance, of variants of the CATS framework, e.g. Delli Gatti et al. (2010).

${ }^{20}$ To be precise, also in the case of networked MABMs, the network can be rewired. Typically, with a certain probability and a certain periodicity, an agent switches from one partner to another.
} 
consists of bank deposits only.

In LEN, households are linked in a network of trading relationships to a finite set of firms from which they buy C-goods and firms for which they work. Since there are no banks, households hold wealth in liquid form (money holding).

In AGH, each household ("person") is denoted by the type $(i, j)$ where $i$ is the household's labor/product type and $j$ are the types of goods the household wants to consume. By assumption the household consumes only two goods, different from the good she can produce. The product type is isomorphic to the labour type. ${ }^{21}$ A household of type $i$ can be a worker if employed by a firm ("shop") of the same type. If a worker, she earns a wage. Otherwise, the household can be a firm owner.

In the CATS framework, households can be either workers or "capitalists". Workers supply labor, earn a wage (if employed), consume and save. Capitalists are the owners of firms. For simplicity there is one capitalist per firm. Capitalists earn dividends (if the firm is profitable), consume and save (therefore they behave as rentiers). Both workers and capitalists accumulate their savings in the form of deposits at banks. If the firm goes bankrupt, the owner of the bankrupt firm employs his personal wealth to provide equity to the entrant firm. In other words, the capitalist is de facto re-capitalizing the defaulting firm to make it survive.

In KS, EUBI, EUGE and LAGOM each household supplies labor and owns firms at the same time. This alternative approach poses the problem of attributing ownership rights, dividends and recapitalization commitments to heterogeneous households. For instance, in EUBI, the household holds financial wealth in the form of deposits at banks and an index of stocks which define property rights and the distribution of dividends.

In JAMEL, some of the households (chosen at random) are firm owners and remain firm owners for a certain time period (typically a run of a simulation).

\subsubsection{The demand for consumption goods}

In this section we will first recall the basic tenets of the standard model of household's consumption/saving decisions, which we will refer to as the Life Cycle/Permanent Income (LCPI) benchmark. We will then present the most general specification of the consumption behavioral rule which we can extract from the MABM literature. The specific behavioral rules adopted by different MABMs can be conceived as special cases of this general specification.

The Life Cycle/Permanent Income benchmark The standard approach to households' behavior (incorporated in NK-DSGE models) is based on "two-stage budgeting". In the first stage, the representative infinitely lived household maximizes expected lifetime utility subject to the intertemporal budget constraint, determining the optimal size of consumption expenditure $C_{h, t}$, which we will sometimes refer to hereafter as the consumption budget.

In the second stage the household determines the composition of $C_{h, t}$, i.e., the fraction $C_{i, h, t} / C_{h, t}$ for each variety $i=1,2, . ., F_{c}$ where $F_{c}$ is the cardinality of the set of C-goods (and of C-firms). ${ }^{22}$

\footnotetext{
${ }^{21}$ In other words, technology is one-to-one. Each household is endowed with a unit of specific labor - say, labor of the i-th type - so that she can produce one unit of the i-th product.

${ }^{22}$ By construction, in a Dixit-Stiglitz setting $C_{h, t}$ is a CES aggregator of individual quantities.
} 
As far as the first stage is concerned, optimal consumption turns out to be a function of expected future consumption $E_{t} C_{h, t+1}$ and the real interest rate $r$ (consumption Euler equation).

Notice now that consumption expenditure is equal by definition to permanent income: $C_{h, t}=Y_{h, t}^{p}$. Hence, after some algebra, we get

$$
C_{h, t}=\hat{r}\left(W_{h, t}^{h}+W_{h, t}^{f}\right)
$$

where $\hat{r}=\hat{R}-1$ is the (net, real) interest rate, $W_{h, t}^{h}$ is human capital and $W_{h, t}^{f}$ is financial wealth. Equation (1) can be interpreted as a benchmark Life Cycle/Permanent Income (LCPI) consumption function. In this framework, by construction, the consumption budget is equal to the annuity value of total (financial and human) wealth.

Human capital, in turn, is defined as the discounted sum of current income and expected future incomes accruing to the household:

$$
W_{h, t}^{h}=\frac{1}{\hat{R}} \sum_{s=0}^{\infty}\left(\frac{1}{\hat{R}}\right)^{s} E_{t} Y_{h, t+s}=\frac{1}{\hat{R}} Y_{h, t}+\frac{1}{\hat{R}} \sum_{s=1}^{\infty}\left(\frac{1}{\hat{R}}\right)^{s} E_{t} Y_{h, t+s}
$$

Substituting (2) into (1), the LCPI consumption function becomes:

$$
C_{h, t}=\frac{\hat{r}}{\hat{R}} Y_{h, t}+\frac{\hat{r}}{\hat{R}} \sum_{s=1}^{\infty}\left(\frac{1}{\hat{R}}\right)^{s} E_{t} Y_{h, t+s}+r W_{h, t}^{f}
$$

In words: consumption is a linear function of current and expected future incomes and of financial wealth.

By definition, in the LCPI benchmark, $W_{h, t+1}^{f}=\hat{R} W_{h, t}^{f}+Y_{h, t}-C_{h, t}$. Therefore saving - i.e. the change in financial wealth $S_{h, t}=W_{h, t+1}^{f}-W_{h, t}^{f}-$ turns out to be equal to $S_{h, t}=$ $\hat{r} W_{h, t}^{f}+Y_{h, t}-C_{h, t}$. Since consumption is equal to permanent income, saving can be specified as follows:

$$
S_{h, t}=\hat{r} W_{h, t}^{f}+\left(Y_{h, t}-Y_{h, t}^{p}\right)
$$

where the expression in parentheses is transitory income. All the income in excess of permanent income will be saved and added to financial wealth. If, on the other hand, current income falls short of permanent income, the household will stabilize consumption by decumulating financial wealth.

As far as the second stage is concerned, it is easy to show that in a Dixit-Stiglitz framework the (optimal) fraction of each good in the bundle is

$$
\frac{C_{i, h, t}}{C_{h, t}}=\left(\frac{P_{i, t}}{P_{t}}\right)^{-\varepsilon}
$$

where $P_{i, t}$ is the price of the i-th variety, $P_{t}$ is the general price level ${ }^{23}$ and $\varepsilon$ is the absolute value of the price elasticity of demand. In words: the fraction of the consumption budget allocated to each variety is a decreasing function of the relative price $\frac{P_{i, t}}{P_{t}}$.

Generally, households are assumed to be identical. If household members are heterogeneous (for instance because of the employment status, or the level and the source of income),

\footnotetext{
${ }^{23}$ By construction, the general price level is a CES aggregator of individual prices.
} 
in standard models complete markets are assumed so that idiosyncratic risk can always be insured. Within the household, "full consumption insurance" follows from the assumption that household members pool together their incomes (wages, unemployment subsidies, dividends) and consume the same amount in the same proportions. Thanks to this assumption, heterogeneity, albeit present, is irrelevant because the household may still be dealt with as a unique (representative) agent. If idiosyncratic income risk is uninsurable, then heterogeneity cannot be assumed away. ${ }^{24}$

The Agent Based approach to consumption/saving decisions A two stage procedure is also generally adopted in MABMs. In the first stage household $h$ determines the consumption budget $C_{h, t}$, i.e., the amount of resources (income, wealth) to be allocated to consumption expenditure. In the second stage the household determines the composition of the bundle of consumption goods, i.e., the quantities $C_{i, h, t}, i=1,2, \ldots F_{c}$ of the goods which enter the consumption bundle. Notice however that, in general, agents do not follow explicit optimization procedures. Markets are generally incomplete and within-household consumption insurance is ruled out.

\section{The choice of the consumption budget}

As far as the first stage is concerned, the most general behavioral rule adopted to set the consumption budget in the MABM literature can be specified as follows:

$$
C_{h, t}=c_{h} W_{h, t}^{h}+c_{f} W_{h, t}^{f}
$$

where $W_{h, t}^{f}$ is the household's financial wealth (deposited at the bank and/or invested in financial assets), $W_{h, t}^{h}$ is human capital, $c_{h}$ and $c_{f}$ are propensities to consume, both positive and smaller than one. In words, consumption is a linear function of human and financial wealth.

By definition $W_{h, t}^{f}=\hat{R} W_{h, t-1}^{f}+Y_{h, t}-C_{h, t}$ and $S_{h, t}=W_{h, t}^{f}-W_{h, t-1}^{f}$. Consumption is defined as in (6). Hence, in a generic MABM savings turn out to be:

$$
S_{h, t}=Y_{h, t}+\left(\hat{r}-c_{f}\right) W_{h, t-1}^{f}-c_{h} W_{h, t}^{h}
$$

If positive, savings increase financial wealth. In some MABMs saving can be involuntary: it may happen that the household cannot find enough consumption goods at the limited number of firms she visits. Saving will turn negative - i.e. the household will decumulate financial wealth - if the consumer does not receive income - for instance because a worker becomes unemployed and/or financial income (interest payments on financial wealth) is too low. In most MABMs household do not get into debt so that consumption smoothing is limited or absent (in the jargon of NK-DSGE models, asset market participation is limited).

Specifications of the general rule (6) differ from one MABM to the other.

AGH set $c_{h}=c_{f}=c$. Moreover, they define human capital as the capitalized value of permanent income $W_{h, t}^{h}=k^{h} Y_{h, t}^{p}$ where $k^{h}$ is a capitalization factor ${ }^{25}$. Permanent income

\footnotetext{
${ }^{24}$ Incomplete markets is the basic assumption of the literature on Standard Incomplete Market (SIM) models with heterogeneous agents, both of the new Classical and New Keynesian type. The New Keynesian variants are known as Heterogeneous Agents New Keynesian (HANK) models. For an exhaustive survey, see the chapter by Ragot in this Handbook.

${ }^{25} \mathrm{in} \mathrm{AGH} k^{h}$ is endogenous as it is a function of projections of inflation and the interest rate elaborated by the central bank and made available to the general public.
} 
$Y_{h, t}^{p}$ is computed by means of an adaptive algorithm: $Y_{h, t}^{p}-Y_{h, t-1}^{p}=(1-\xi)\left(Y_{h, t-1}-Y_{h, t-1}^{p}\right)$ where $\xi \in(0,1)$ is a memory parameter. By iterating, it is easy to see that permanent income (and therefore human capital) turns out to be a weighted sum of past incomes only: $Y_{h, t}^{p}=(1-\xi) \sum_{s=0}^{-\infty} \xi^{s} Y_{h, t-s-1}$. Hence the AGH behavioral rule for consumption expenditure is

$$
C_{h, t}=c k^{h}(1-\xi) \sum_{s=0}^{-\infty} \xi^{s} Y_{h, t-s-1}+c W_{h, t}^{f}
$$

In words, consumption is a linear function of past incomes and financial wealth.

In the CATS/ADG framework, human capital is defined by the following adaptive algorithm: $W_{h, t}^{h}=\xi W_{h, t-1}^{h}+(1-\xi) Y_{h, t}$. By iterating, one gets $W_{h, t}^{h}=(1-\xi) Y_{h, t}+(1-$ $\xi) \sum_{s=1}^{-\infty} \xi^{s} Y_{h, t-s}$ i.e., human capital is a weighted average of current and past incomes. ${ }^{26}$ Substituting this definition into (6) the behavioral rule specializes to

$$
C_{h, t}=c_{h}(1-\xi) Y_{h, t}+c_{h}(1-\xi) \sum_{s=1}^{-\infty} \xi^{s} Y_{h, t-s}+c_{f} W_{h, t}^{f}
$$

While human capital in the neoclassical approach is a linear combination of current and expected future incomes, and therefore is formed in a forward looking way, the proxy for human capital in AGH and CATS/ADG is a linear combination of current and past incomes, i.e. it is determined by a backward looking algorithm. ${ }^{27}$

Setting $\xi=0$ - i.e., assuming that there is no memory - human capital boils down to current income, so that (9) specializes to:

$$
C_{h, t}=c_{y} Y_{h, t}+c_{f} W_{h, t}^{f}
$$

where $c_{y}$ is the propensity to consume out of income. ${ }^{28}$

Setting $c_{f}=0$, from (10) we get a specification of the consumption function sometimes adopted in MABMs of a strictly Keynesian flavor

$$
C_{h, t}=c_{y} Y_{h, t}
$$

In many models of the $\mathbf{K S}$ family, the behavioural rule is (11) with $c_{h}=1$ :

$$
C_{h, t}=Y_{h, t}
$$

This specification describes the behaviour of "Hand to mouth" consumers. ${ }^{29}$

\footnotetext{
${ }^{26} \mathrm{In}$ ADG income accruing to the household is $Y_{h, t}=w$ if the consumer is a worker with an active labor contract, $Y_{f, t}=\tau \pi_{f, t-1}$ if the consumer is a capitalist receiving dividends; $\tau$ is the dividend-payout ratio and $\pi_{f, t-1}$ are profits of the firm realized in the previous period and accruing as income to the capitalist in the current period.

${ }^{27}$ This difference reflects the fact that forward looking expectation formation is marred with insurmountable difficulties in a complex heterogeneous agents context.In this context, the obvious candidate for expectation formation is an adaptive algorithm. Notice, however, that the empirical work on consumption has extensively used adaptive algorithms.

${ }^{28} c_{y}$ in (10) coincides with $c_{h}$ in (9) because, in the absence of memory, human capital coincides with current income.

${ }^{29}$ This is the specification used in NK models with "Rule of thumb" consumers, i.e. consumers who cannot smooth consumption over the life cycle due to "limited asset market participation" or "liquidity constraints".
} 
LAGOM adopts a rule such as (10) with $c_{y}=0$. Moreover, since financial wealth coincides with money holding $\left(W_{h, t}^{f}=\frac{M_{h, t}}{P_{t}}\right)$, the consumption budget turns out to be an increasing linear function of real money balances: ${ }^{30}$

$$
C_{h, t}=c_{f} \frac{M_{h, t}}{P_{t}}
$$

In CATS/DD, consumption is a special case of (10) obtained by setting $c_{y}=c_{f}=c$. Therefore:

$$
C_{h, t}=c\left(Y_{h, t}+W_{h, t}^{f}\right)
$$

The expression in parentheses is one of the possible specifications of "cash on hand". Cashon-hand can be defined in the most general way as liquid assets which can be used to carry on transactions. In small MABMs which do not feature banks such as LEN, cash-on-hand coincides with currency. In a setting with banks such as CATS/DD, cash on hand coincides with new deposits, which in turn amount to the sum of income and old deposits. This is in line with Deaton (1991)), who defines cash on hand as the sum of income and financial assets.

In EUBI and EUGE saving behavior aims at achieving a target for wealth: $S_{h, t}=$ $\nu\left(\omega^{f} Y_{h, t}-W_{h, t}^{f}\right)$ where $\omega^{f}$ is the target wealth-to-income ratio and $\nu$ is the velocity of adjustment of wealth to targeted wealth. Since, by definition $S_{h, t}=Y_{h, t}-C_{h, t}$, simple algebra shows that under this assumption the consumption budget can be written as:

$$
C_{h, t}=\left(1-\nu \omega^{f}\right) Y_{h, t}+\nu W_{h, t}^{f}
$$

which is a special case of (10) with $c_{y}=1-\nu \omega^{f}$ and $c_{f}=\nu$.

Carroll has shown that in an uncertain world consumption is a concave function of cashon-hand which he defines as the sum of human capital and beginning of period wealth. ${ }^{31}$ In Carroll's framework, for low values of wealth, the propensity to consume (out of wealth) is high due to the precautionary motive: a reduction of wealth would in fact lead to a sizable reduction of consumption to rebuild wealth (buffer stock rule). In some MABMs the consumption function is adjusted to mimic Carroll's precautionary motive, i.e., to reproduce the non linearity of the relationship between consumption and wealth. This theoretical setting has been corroborated by a number of empirical studies (see Carroll and Summers (1991), Carroll (1997)), such that using this approach in MABMs is consistent with the agenda to rely on behavioral rules which have strong empirical foundations.

For instance in EUBI, the specification of the consumption function discussed above gives rise to a piecewise linear heuristic based on the buffer-stock rule. Also EUGE adopt a specification of the consumption budget which adopts Carroll's rule.

In the CATS/MBU framework consumption is specified as in (11) but $c_{y}$ is a non-linear function of wealth. This allows to capture the interaction between income and wealth in the determination of consumption.

\footnotetext{
${ }^{30}$ In LAGOM firms enter the market for C-goods to purchase raw materials and wholesale goods (circulating capital). The demand for C-goods of the i-th firm is $K_{i, t}^{c}=\left(1 / k^{c}\right) Y^{T}-K_{i, t-1}^{c}$ where $Y^{T}$ is the production target of the firm, $k^{c}$ is the marginal productivity of circulating capital and $K_{i, t-1}^{c}$ is the stock of circulating capital inherited from the past.

${ }^{31}$ See Carroll $(1992,1997,2009)$.
} 
LEN explicitly models the consumption budget as an increasing concave function of money holdings:

$$
C_{h, t}=\left(\frac{M_{h, t}}{P_{t}}\right)^{c}
$$

where $c \in(0,1) .{ }^{32}$

JAMEL presents a variant of Carroll's framework. The household computes "average income" (which can be considered a proxy of permanent income) as the mean of incomes received over a certain time span occurred in the past: $Y_{h, t}^{a}=\frac{1}{n} \sum_{\tau=t-n}^{t} Y_{h, \tau}{ }^{33}$ Moreover, JAMEL define desired or targeted cash on hand as a fraction of average income: $m_{h, t}^{T}=s Y_{h, t}^{a}$, where $m_{h, t}^{T}=M_{h, t}^{T} / P_{t}$ are real money balances and $s \in(0,1)$ is the desired propensity to save (out of average income). The consumption budget is defined as follows:

$$
C_{h, t}= \begin{cases}c Y_{h, t}^{a} & \text { if } m_{h, t}<m_{h, t}^{T} \\ Y_{h, t}^{a}+c_{m}\left(m_{h, t}-m_{h, t}^{T}\right) & \text { if } m_{h, t}>m_{h, t}^{T}\end{cases}
$$

where $c=1-s$ and $c_{m} \in(0,1)$ is the propensity to consume. In words: if liquid assets are (relatively) "low", the household spends a fraction $c$ of average income. If liquidity is "high", the household consumes the entire average income and a fraction $c_{m}$ of excess money balances, defined as the difference between the current and targeted money holding.

With the help of some algebra, the behavioral rule above can be written as follows:

$$
C_{h, t}= \begin{cases}c Y_{h, t}^{a} & \text { if } Y_{h, t}^{a}>\bar{Y}_{h, t}^{a} \\ c^{\prime} Y_{h, t}^{a}+c_{m} M_{h, t} & \text { if } Y_{h, t}^{a}<\bar{Y}_{h, t}^{a}\end{cases}
$$

where $\bar{Y}_{h, t}^{a}=\frac{1}{s} m_{h, t}$ is the cut-off value of average income and $c^{\prime}=1-c_{m}(1-c)=\left(1-c_{m}\right)(1-$ $c)+c$. Notice that $c^{\prime}>c$. The cut-off value of average income is a multiple of current money holdings. The specification above highlights the basic tenets of Carroll's buffer stock theory in a very simple piece-wise linear setting. When average income is below the threshold - i.e. when the threshold is relatively high because the household is wealthy/liquid - the marginal propensity to consume is $c^{\prime}$ i.e., it is relatively high. When average income surpasses the threshold the marginal propensity drops to $c$.

JAMEL also incorporates consumer sentiment and opinion dynamics in the consumption function. The propensity to save $s$ can take on two values. When "optimistic", the household is characterized by $s^{L}$ while if "pessimistic" she sets s to $s^{H}$, with $s^{H}>s^{L}$. Pessimism leads to a reduction of the propensity to consume: households save more for rainy days.

Each household switches from a low to a high propensity to save (and vice versa) depending on her employment status. The household turns pessimistic if unemployed. In each period, the household observes the consumer sentiment (proxied by the employment status) of a finite subset of other households (a neighborhood for short). With probability $\pi^{m}$ she adopts the majority opinion - i.e. the prevailing consumer sentiment - of the neighborhood; with probability $1-\pi^{m}$ the household relies on her own situation: if she is unemployed

\footnotetext{
${ }^{32}$ Of course, consumption cannot be greater than money holding. Hence $\left(M_{h, t} / P_{t}\right)>1$

${ }^{33} \mathrm{In}$ JAMEL the minimal time unit is a month. $Y_{h, t}$ therefore is current income in month $\mathrm{t}$. Average income in month $\mathrm{t}$ is the mean of monthly incomes in the last 12 months up to t, i.e. $Y_{h, t}^{a}=\frac{1}{12} \sum_{\tau=t-12}^{t} Y_{h, \tau}$. Since permanent income is the weighted average of all the past incomes over an infinite time span with exponentially decaying weights (see above), average income is defined on a shorter time span and with equal instead of decaying weights.
} 
(employed), she will be pessimistic (optimistic) and will choose $s^{H}\left(s_{L}\right)$. The probability of adopting the sentiment of the majority can be interpreted as the strength of the households' "animal spirits".

The choice of the goods to buy As to the second stage, the choice of the goods to buy is generally influenced by the relative price. EUBI and EUGE introduce a multinomial logit model, CATS posit a search mechanism.

In LEN each household is linked to a fixed number of firms from which she can buy. The network can change over time. Each period, with a certain probability, the household chooses at random a firm in the neighborhood of current trading partners and a firm outside it, compares the prices and switches to the new firm if the price of the price set by the current partner exceeds the price of the new firm by at least a certain margin. In the end, therefore, the household searches for better trading opportunities based on relative price. ${ }^{34}$

AGH posit that the household can buy only from two firms ("stores", i.e., shops where the household purchases her consumption goods). Hence she has to choose the quantity to be demanded from each firm. AGH derives behavioral rules by solving a simple optimization problem. The household maximizes $U_{h, t}=U\left(C_{1, h, t}, C_{2, h, t}\right)$ subject to $p_{1, t} C_{1, h, t}+p_{2, t} C_{2, h, t}=$ $C_{h, t}$ where $p_{i, t}=\frac{P_{i, t}}{P_{t}}, i=1,2$. Hence the quantity demanded for each variety turns out to be:

$$
C_{i, h, t}=f_{i}\left(p_{1, t}, p_{2, t}, C_{h, t}\right) i=1,2
$$

which can be easily compared with (22). The household explores the goods market looking for stores supplying the goods she wants to consume. The household buys from store $\mathrm{j}$ only if the posted price $P_{j, t}$ is smaller than a reservation price equal to the price paid by the household in the past $P_{h, j, t-1}$ (appropriately indexed): $P_{h, i, t-1}\left(1+\pi^{T}\right)>P_{j, t}$ where $\pi^{T}$ is expected inflation, anchored to the inflation target of the central bank. ${ }^{35}$

In the CATS framework, households collect information and pick goods by visiting firms chosen at random. The h-th household visits $Z_{c}$ randomly selected firms, ranks them according to the price they charge and purchases C-goods starting from the firm charging the lowest price. If she does not spend the entire consumption budget at the first firm, she will move up to the second firm in the ranking and so on. If she has not spent all the consumption budget after visiting $Z_{c}$ firms, she will save involuntarily. This implies that the demand for the good produced by the i-th C-firm is implicitly decreasing with the relative price $p_{i, t}$. Notice however that the implicit demand curve that the i-th firm is facing is shifting over time. A given firm may be visited by different sets of consumers on subsequent days. The search and matching mechanism leads to the coexistence of queues of unsatisfied consumers (involuntary savers) at some firms and involuntary inventories of unsold goods at some other firms.

LAGOM adopts a similar market protocol as CATS for the market for C-goods. In the LAGOM model also firms enter the market for C-goods as they use circulating capital together with fixed capital for production.

EUBI and EUGE assume that the consumer receives information about the range of products, their prices and their availability at (local) malls. The choice of the good to buy

\footnotetext{
${ }^{34}$ The household leaves the current partner and switches to a different one also when the household is demand constrained, i.e., when current trading partners are unable to satisfy demand.

${ }^{35}$ Of course, this assumption is implicitly based on the credibility of the central bank's announced inflation target.
} 
is random and the probability of buying a certain product is determined by a multinomial logit model:

$$
P[\text { consumer h selects good i }]=\frac{\exp \left(-\gamma \log P_{i, t}\right)}{\sum_{j} \exp \left(-\gamma \log P_{j, t}\right)}
$$

where the sum in the denominator includes all products for which the consumer has received information. ${ }^{36}$ As has been discussed in Dawid et al. (2018c) this formulation can also be interpreted as a representation of a situation in which the product offered by the different C-firms are horizontally differentiated along dimensions not explicitly captured in the model. The parameter $\gamma$ governs the intensity of (price) competition between the C-firms and typically has strong influence on the emerging economic dynamics on the aggregate level. Dawid et al. (2018c) also point out that the use of logit-choice models for the representation of consumer choice has strong empirical foundations, e.g. in the Marketing literature. ${ }^{37}$

\subsubsection{Labour supply}

On the labour market, households are suppliers of labor. The h-th worker supplies inelastically one unit of labor.

LEN assumes that each household has a reservation wage, denoted with $w_{h}$. She supplies her labor only to a finite subset of the population of firms, a neighborhood of potential employers. However, the network can be re-wired. If the household is employed, say, at firm $i$, and her current wage is higher than the reservation wage $\left(w_{i}>w_{h}\right)$, she will search for a better position infrequently, i.e. with probability less than one. When searching, she will visit only one new firm chosen at random. If at this new firm, say $j$, she finds $w_{j}>w_{i}$, she will quit the current job and move to the j-th firm. If, for any reasons, her current wage falls below the reservation wage $\left(w_{i}<w_{h}\right),{ }^{38}$, she will intensify the quest for a better position by exploring a new firm every period (i.e., with certainty). If the household is unemployed, she will search for a job every period by visiting a fixed number $Z_{e}$ of firms chosen at random. If the household switches to a new employer, the former employer drops out of the set of employers of the households and is replaced by the new one.

Also in LAGOM each household has a reservation wage. If the household is employed, say, at firm $i$, and her current wage is lower than the reservation wage $\left(w_{i}<w_{h}\right)$, she will quit her job and search for a better position at a finite set of firms chosen at random.

In AGH, as we saw above, a household type is defined by the production good it can produce with its labor type. If employed, the household is working in the same firm which produces her production good. If unemployed, the household engages in job search with a given probability $\pi^{s}$. She explore the labor market looking for a firm (with the same product type) posting a vacancy. The household accepts the job offer if her previous wage $w_{h, i, t-1}$ (appropriately indexed) is smaller than the posted wage $w_{i, t}$, i.e., if $w_{h, i, t-1}\left(1+\pi^{T}\right)<w_{i, t}$.

In CATS/MBU and CATS/ADG, the h-th household, if unemployed, looks for a job by visiting $Z_{e}$ firms chosen at random and applying to those with open vacancies. In CATS/MBU the posted wage is firm-specific and cannot drop below a lower bound represented by a minimum wage, which is changing over time due to indexation. The unemployed

\footnotetext{
${ }^{36}$ In Dawid et al. (2018b) this setting is extended by assuming that consumers have a 'home-bias' in consumption, such that the attractiveness of a product depends in addition to the price also on the fact whether it has been produced by a firm located in the same region as the consumer.

${ }^{37}$ An axiomatic behavioral foundation for multinomial logit models can be found in Breitmoser (2016).

${ }^{38}$ This case may be due to the decision of the firm to cut the posted wage
} 
worker will accept a job from the firm with open vacancies which pays the higher wage. For simplicity in CATS/ADG the posted wage is given and uniform across firms so that the unemployed worker accept the job at the first chance of finding an open vacancy. If she doesn't find an open vacancy, the household remains unemployed and funds her consumption by dis-saving, i.e. consuming out of accumulated wealth. For simplicity CATS assume that there are neither hiring nor firing costs. If a firm wants to scale down activity in a certain period, it can fire workers at no costs. Fired workers become unemployed and start searching for a job in the same period.

In EUBI the household is characterized by a specific skill and has a reservation wage. If unemployed, she explores the labor market with a given probability. Firms post vacancies and wages. The unemployed household ranks the wage offers and applies only to firms that make a wage offer (for the worker's skill group) which is higher than her reservation wage. If the unemployed job searcher receives several acceptable job offers she accepts the offer with the highest wage. The reservation wage of the worker is adjusted downwards during periods of unemployment.

In JAMEL, the household's reservation wage is stochastically reduced if the unemployment spell is greater than an exogenous upper bound. In symbols:

$$
w_{h, t}= \begin{cases}w_{h, t-1}\left(1-\eta^{h}\right) & \text { if } d_{h, t}^{u}>\bar{d}^{u} \\ w_{h, t-1} & \text { if } d_{h, t}^{u}<\bar{d}^{u}\end{cases}
$$

where $\eta^{h} \geq 0$ is a random draw from a uniform distribution, $d_{h, t}^{u}$ is the duration of the unemployment spell and $\bar{d}^{u}$ is the threshold value of this spell.

\subsubsection{The demand for financial assets}

In a small MABM such as LEN, households can hold wealth only in the most liquid form (cash). Most medium-sized MABMs assume that there is at least one other financial asset such as deposits at banks. For CATS, KS, JAMEL, LAGOM households' wealth consists only of deposits. In AGH, households can hold cash, deposits and Government bonds. In EUBI and EUGE the household can hold wealth as liquidity (deposits at banks), as a portfolio of shares issued by firms and banks and in EUGE also as government bonds. Households therefore, receive dividends according to the composition of their portfolio of shares. For simplicity, in EUBI the allocation of households' savings to bank deposits and shares is random. Modeling the portfolio choice for each household, however, may be quite complex. For instance, the preference structure of "investors" in EUGE - i.e., households who trade in financial markets - takes into account insights from behavioral finance, namely myopic loss aversion. Transactions on financial markets occur at higher frequency than on real markets (daily as against monthly). Prices are determined in equilibrium through a clearinghouse mechanism. In CATS households can be of two types, workers and capitalists. Assuming that there is one capitalist per firm, each firm distributes part of its profits to the firm owner as dividends. KS adopt the extreme assumption that all the profits are retained within the firm and used to accumulate net worth. In this case, by assumption there will be no dividends. In JAMEL some households (chosen at random) are firm owners and therefore receive dividends which will be distributed only if the firm's net worth is higher than a threshold (defined by a target level for leverage). Opinion dynamics play a role in dividend distribution. Firms switch from "optimistic", (characterized by high target leverage and 
high dividends) to "pessimistic" (low target leverage and low dividends). Also in LAGOM households are firm owners and receive both wages and dividends.

\section{$2.4 \quad$ Firms}

In this section we will consider a population of $F$ firms, which produce either consumption goods (C-firms) or capital goods (K-firms). Variables pertaining to the i-th $\mathrm{C}$-firm will be denoted with the index $i=1,2, \ldots F_{c}$. Variables pertaining to the $\mathrm{k}$-th $\mathrm{K}$-firm will be denoted with the index $k=1,2, \ldots F_{k}$. In most MABMs C-firms demand labor and K-goods in order to produce and sell C-goods to households; K-firms demand labor in order to produce and sell K-goods to C-firms. ${ }^{39}$.

Firms have market power, hence they set the quantity and the price of the goods they produce and sell. Generally, market power is rooted in transaction costs. Due to uncertainty and the costs of exploring market conditions, in fact, buyers do not have enough information on the prices and quantities set by all the sellers so that they purchase goods from a limited set of suppliers. Each supplier, therefore, operates in condition of monopolistic competition or oligopoly on the market for her own good.

The planned (or desired) scale of activity is the main determinant of the demand for inputs, namely the demand for capital and the demand for labor.

On the market for K-goods, demand comes from C-firms and supply comes from K-firms. The i-th C-firm's demand for K-goods - i.e. investment - is driven mainly by production requirements. If production planned in $\mathrm{t}$ for $\mathrm{t}+1$ can be carried out with the capital stock inherited from the past (after depreciation), the firm simply adjusts capacity utilization. If capital inherited from the past is insufficient to undertake planned production, the i-th firm should expand capacity by purchasing new K-goods (sometimes referred to as "machine tools"). In some MABMS, this is feasible. In other MABMs, investment in $\mathrm{t}$ is driven by long run production requirements and cannot be used to face short run peaks of demand. Hence the firm will be constrained to produce at full capacity but less than desired.

On the market for labour, demand comes from all the firms and supply comes from households. Firms whose workforce is insufficient to undertake planned production will post vacancies and a wage offer (which may be firm-specific or uniform across firms, constant or time-varying because of indexation and/or because of labour market conditions.)

Firms may have a financing gap, i.e., a positive difference between operating costs and internally generated funds. They generally ask for a loan to fill this gap. The quantity and price of credit are set by banks.

Firms may be unable to achieve the desired scale of activity for lack of funds (if they are rationed on the credit market), of capital (if investment is disconnected from short run production need and/or if they don't find enough machine tools to buy) or of labor (if they don't find enough workers to hire).

\subsubsection{The supply of consumption goods}

The Dixit-Stiglitz benchmark As far as the corporate sector is concerned, NK DSGE models are based on monopolistic competition: each firm behaves as a monopolist on her own market. The firm's market power is due to product heterogeneity. Products may be different

\footnotetext{
${ }^{39}$ In some MABMs, firms use also raw materials, energy and wholesale/intermediate goods as inputs. We will be more specific below.
} 
in the eyes of consumers for a number of reasons: spatial dispersion, product differentiation or transaction costs which may generate captive markets.

In the Dixit-Stiglitz benchmark, the demand of the h-th household for the i-th good is represented by equation (5). Summing across $H$ households and rearranging one gets the demand function for the i-th good:

$$
C_{i, t}=\left(\frac{P_{i, t}}{P_{t}}\right)^{-\varepsilon} \frac{C_{t}}{F_{c}}
$$

where $C_{t}=\sum_{h=1}^{H} C_{h, t}$ is total consumption (economywide). ${ }^{40}$

With a linear technology and only the labour input, the marginal cost will be $w_{t} / \alpha$ where $w_{t}$ is the wage and $\alpha$ is the marginal productivity of labor. Optimal pricing yields

$$
P_{i, t}^{*}=(1+\mu) \frac{w_{t}}{\alpha}
$$

where $\mu=(\varepsilon-1)^{-1}$ is the mark-up.

Substituting (23) into (22) one gets the optimal scale of production:

$$
Y_{i, t}^{*}=C_{i, t}^{*}=\left(\frac{1+\mu}{\alpha} \frac{w_{t}}{P_{t}}\right)^{-\varepsilon} \frac{C_{t}}{F_{c}}
$$

Since the manager of the firm knows the production function and input costs, and therefore can compute the marginal cost, if she knows also the demand curve she will choose an optimal mark up (i.e., ratio of the individual price to marginal cost) and an optimal scale of activity. In other words, in the absence of uncertainty on market demand, the firm will choose a point on the demand curve in such a way as to maximize profits. In this case supply will always be equal to demand: the firm will neither accumulate involuntary inventories of unsold goods nor face queues of unsatisfied consumers: output will always be sold and no potential customer will leave the firm's premises empty handed.

Moreover, in the absence of frictions on the labour market, the firm will always be able to carry out planned production. In the end therefore planned output will be equal to actual output and actual output will be equal to sales.

From (23) and (24) we can predict the following: the firm will react to (i) an increase of elasticity of demand by reducing the mark up and therefore the individual price and increasing production; (ii) an increase of the real wage by increasing the price and reducing production; (iii) an increase in productivity by reducing the price and increasing production, (iv) an increase in total consumption by increasing production.

Since firms have the same technology, incur the same labour cost and face the same demand function, a symmetric equilibrium in which all firms charge the same price, is considered: $P_{i, t}=P_{t}$, so that the real wage will be equal to $\left(w_{t} / P_{t}\right)^{*}=\alpha /(1+\mu)$. At that wage, employment is determined by labor supply, the labour market clears and there is full employment. ${ }^{41}$ Total output is equal to the full employment level of GDP. ${ }^{42}$ In the absence

\footnotetext{
${ }^{40}$ For the sake of comparison with MABMs, equation (22) concerns the case of a "large" but finite number of firms. Equation (5) is derived assuming a continuum of firms of unit mass.

${ }^{41}$ In the standard model labor supply derives from expected lifetime utility maximization when leisure is an argument of the period utility function. It turns out that labor supply is increasing with the real wage. The real wage in the symmetric equilibrium with monopolistic competition is smaller than in perfect competition, because in the latter case the mark up is zero. Hence full employment with monopolistic competition will be smaller than in the case of perfect competition.

${ }^{42}$ This is the characterization of total output in the equilibrium with flexible prices of the standard NK DSGE model.
} 
of investment, output is equal to consumption. Each firm therefore will produce the same quantity, i.e., a fraction $1 / F_{c}$ of total consumption, which is equal to total full employment output. In the presence of nominal rigidities (e.g. Calvo pricing), this level of output is unattainable, at least in the short run.

Price/quantity decisions when demand is unknown In order to understand the price/quantity strategies of firms in MABMs and compare them with firms' decisions in the standard model, in this "intermezzo" we will keep the basic structure of the Dixit-Stiglitz framework but will relax the assumption of perfect information as far as market demand is concerned. We will assume instead that, at the moment decisions on price and quantity must be made, the firm does not know the position of the demand curve on the price/quantity space.

Uncertainty on market demand may be due to the fact that transactions do not occur at the same time on all markets. There is a sequence of events which take place at different times on different markets. For instance we can envisage the following 4 steps. (1) At the beginning of period $t$ (before the market for C-goods opens), the firm decides the price and the quantity. On the basis of the production plan, it decides whether to hire or fire workers. Suppose the workforce must be expanded to fulfill the production plan. (2) The firm enters the labor market by posting vacancies at a certain wage. These vacancies may or may not be filled depending on the number of matches between demand (job offers) and supply (unemployed workers) of labor. Suppose all the vacancies are filled. (3) The firm can therefore implement the desired production plan. (4) The quantity produced is then sold on the market of C-goods. Production must be decided in step (1) but actual demand will be revealed to the firm only in step (4).

The information set of the firm at the moment the scale of activity must be decided may contain precise information on the pricing behavior of competitors and the total number of firms but information may be incomplete and imperfect on the price elasticity of demand and/or total households' consumption. In order to maximize expected profits, the firm must estimate the elasticity of demand and total consumption planned by households. The market demand that the i-th firm expects is:

$$
C_{i, t}^{e}=\left(\frac{P_{i, t}}{P_{t}}\right)^{-\varepsilon_{t}^{i}} \frac{C_{t}^{i}}{F_{c}}
$$

where $\varepsilon_{t}^{i}$ and $C_{t}^{i}$ are price elasticity and total consumption expected or estimated by firm i. In order to maximize profits in this informational scenario, the firm could set the price as a mark up over marginal cost, the mark up being a function of the estimated price elasticity:

$$
P_{i, t}=\frac{1+\mu_{t}^{i}}{\alpha} w_{t}
$$

where $\mu_{t}^{i}=\left(\varepsilon_{t}^{i}-1\right)^{-1}$. Plugging (26) into (25) one gets:

$$
C_{i, t}^{e}=\left(\frac{1+\mu_{t}^{i}}{\alpha} \frac{w_{t}}{P_{t}}\right)^{-\varepsilon_{t}^{i}} \frac{C_{t}^{i}}{F_{c}}
$$

The firm uses expected demand to plan production: $Y_{i, t}^{*}=C_{i, t}^{e}$. For the sake of discussion, 
let's assume that actual production is equal to desired production: $Y_{i, t}=Y_{i, t}^{*}$. Hence: ${ }^{43}$

$$
Y_{i, t}=Y_{i, t}^{*}=C_{i, t}^{e}=\left(\frac{1+\mu_{t}^{i}}{\alpha} \frac{w_{t}}{P_{t}}\right)^{-\varepsilon_{t}^{i}} \frac{C_{t}^{i}}{F_{c}}
$$

At the beginning of time (step (1) in the sequence above) $t$ the status quo for the i-th firm is the point $\left(P_{i, t}, Y_{i, t}\right)$ on the price/quantity space. The coordinates are determined by (26) and (28). Once production has been carried out, transactions can take place. Once transactions are completed, the i-th firm can observe the amount of goods actually sold $Q_{i, t}=\min \left(Y_{i, t}, C_{i, t}\right)$ where $C_{i, t}$ is actual demand.

Since sales occur only after the firm has carried out production, actual demand can differ from current production. If $Q_{i, t}=C_{i, t}<Y_{i, t}$, there will be involuntary inventories whose size is: $\Delta_{i, t}=Y_{i, t}-C_{i, t}>0$. This is a signal of excess supply, i.e. of a positive forecasting error (demand has been overestimated).

If $Q_{i, t}=Y_{i, t}<C_{i, t}$, all the output will be sold and therefore there will not be involuntary inventories: $\Delta_{i, t}=0$. The difference $Y_{i, t}-C_{i, t}>0$ measures the demand of unsatisfied consumers, who may turn to other producers (goods are substitutable, albeit only imperfectly) or save involuntarily. This is a signal of excess demand. The firm has made a negative forecasting error (demand has been underestimated).

It can also happen, of course, that demand equals production: $Y_{i, t}=C_{i, t}$. There will be no forecasting error (no inventories and no queues) in this case.

Inventories are defined as:

$$
\Delta_{i, t}=\min \left(Y_{i, t}-C_{i, t}, 0\right)
$$

In the case of excess demand or excess supply the firm is under-performing, ${ }^{44}$ and has an incentive to choose a different price/quantity in the future, say $\left(P_{i, t+1}, Y_{i, t+1}\right)$.

One possible strategy consists in setting the direction of the change in price and quantity in line with the sign of the forecasting error. For instance, if $\Delta_{i, t}>0$ the firm has an incentive to scale down production and cut the price. The size of the change may be linked to the magnitude of the error.

Notice that by making mistakes the firm collects new information on market conditions. As an alternative strategy, the firm can therefore make new estimates of demand and set the price and the quantity accordingly (see equations (26) and (28)).

In the end, uncertainty and imperfect knowledge induce the firm to adapt period by period to evolving market conditions. This adaptation takes the form of price and quantity updating rules. There is no guarantee that this adaptation will lead to the type of equilibrium discussed above.

The Agent Based approach to price/quantity decisions The MABMs we are surveying generally conceive firms as production/trading units immersed in a market environment which is utterly uncertain. Uncertainty is due to the sequential nature of the market economy (which is easily replicated in an agent based framework) and to the ever changing conditions of demand and supply.

\footnotetext{
${ }^{43}$ Notice that, in principle, desired production may not always be carried out because there may be constraints on the attainment of planned production. For instance, in step (2) of the sequence above, not all the vacancies may be filled. Therefore, in step (3) the firm cannot implement the desired scale of activity.

${ }^{44} \mathrm{In}$ other words, the level of profits is smaller than in the optimal position.
} 
Different MABMs deal with price/quantity strategies in different ways. For most of the models we can detect a general pattern, however, in the description of the behavioral rules the firm adopts. ${ }^{45}$ The firm starts with an initial pair of price and quantity, $\left(P_{i, t}, Y_{i, t}\right)$, which we define the status quo. At the end of period $t$, once transactions have been carried out, the firm observes actual sales $Q_{i, t}=\min \left(Y_{i, t}, C_{i, t}\right)$ and learns also the average price $P_{t}$, which is a proxy of the price charged on average by the firms' competitors.

If $Q_{i, t}=C_{i, t}<Y_{i, t}$, there will be an inventory equal to $\Delta_{i, t}=Y_{i, t}-C_{i, t}>0$. Notice that the firm may want to hold a buffer stock of finished goods (desired or voluntary or target inventories). Desired inventories may be an interval: $\Delta_{i, t} \in \Delta_{i, t}^{m}, \Delta_{i, t}^{M}$ - where the extremes are the minimum and maximum levels of desired inventories - or collapse to a single point: $\Delta_{i, t}^{*}$. In the first case there will be a shortage of inventories if $\Delta_{i, t}<\Delta_{i, t}^{m}$ and excess (or involuntary) inventories $\Delta_{i, t}^{u}=\Delta_{i, t}-\Delta_{i, t}^{M}$ if $\Delta_{i, t}>\Delta_{i, t}^{M}$. In the second case there will be a shortage of inventories (resp. involuntary inventories) if $\Delta_{i, t}<\Delta_{i, t}^{*}$ (resp. $\Delta_{i, t}>\Delta_{i, t}^{*}$ ). It is reasonable to assume that planned production for period t will take into account expected demand in the same period and desired inventories.

Of course desired inventories may be zero: $\Delta_{i, t}^{*}=0$. In this case, planned production is equal to expected demand, $Y_{i, t}=C_{i, t}^{e}$, the difference $e_{i, t}=C_{i, t}-Y_{i, t}=C_{i, t}-C_{i, t}^{e}$ therefore is the forecasting error. A positive inventory will always be involuntary and coincide with the absolute value of the (negative) forecasting error: $e_{i, t}<0$ and $\Delta_{i, t}=\left|e_{i, t}\right|>0$. If $Q_{i, t}=Y_{i, t}<C_{i, t}$, the forecasting error is positive $\left(e_{i, t}>0\right)$, there are no inventories $\left(\Delta_{i, t}=0\right)$ but a fringe of unsatisfied consumers.

Desired inventories are generally determined by applying a desired or target inventory-tosales ratio $\delta^{*}$ to current production $\Delta_{i, t}^{*}=\delta^{*} Y_{i, t}$. This ratio represents the number of periods of production at the current rate necessary to build desired inventories. Hence the minimum and maximum levels of voluntary inventories are determined as follows: $\Delta_{i, t}^{m}=\delta_{m} Y_{i, t}$ and $\Delta_{i, t}^{M}=\delta_{M} Y_{i, t}, 0<\delta_{m}<\delta_{M}$. The parameters $\delta_{m}$ and $\delta_{M}$ are the minimum and the maximum inventory-to-sales ratios. If there are no desired inventories, then $\delta^{*}=0$.

The firm receives two types of signals of market conditions: (i) an excess or shortage of inventories (with respect to the target, which may be an interval, a positive value or zero); (ii) the relative price, i.e. the ratio or difference between the individual price and average price. From signals of type (i) the firm infers that it has underestimated or overestimated the strength of demand: a shortage of inventories reveals stronger than expected demand; an involuntary inventory signals weaker than expected demand. From signals of type (ii) the firm infers its position relative to competitors: if the firm's price is lower than the average price, the firm is undercutting competitors (and its market share is bigger than the average market share); if the opposite holds true, the good produced by the firm is overpriced with respect to competitors (and its market share is smaller than the average market share). ${ }^{46}$. The sign and size of signals determine the direction of the price and quantity change to be implemented. The magnitude of the price and quantity change is stochastic and/or governed by the signals. The change in price and quantity therefore will be governed by behavioral rules which take the form of updating algorithms.

The most general form of a quantity updating algorithm we can detect in the MABMs

\footnotetext{
${ }^{45}$ As will become clear below EUBI, EUGE and KS follow approaches somehow different from this general pattern.

${ }^{46}$ The average market share is the share of total consumption that each firm would obtain in a situation of symmetry, i.e. $1 / F_{c}$
} 
under scrutiny is:

$$
Y_{i, t+1}^{*}= \begin{cases}Y_{i, t}\left(1+\eta^{y}\right) & \text { if } \Delta_{i, t}<\Delta_{i, t}^{m} \text { and } P_{i, t}>P_{t} \\ Y_{i, t}\left(1-\eta^{y}\right) & \text { if } \Delta_{i, t}>\Delta_{i, t}^{M} \text { and } P_{i, t}<P_{t}\end{cases}
$$

In words: the firm revises production plans up (resp: down) by $\eta^{y}$ if there is a shortage (excess) of inventories and the current price is greater (smaller) than the competitors' price. The assumption of an upper and a lower bound on the level of desired inventories introduces inertia in quantity updating: if actual inventories fall in the desired interval, the firm does not change the scale of activity.

The most general form of a price updating algorithm is:

$$
P_{i, t+1}^{*}= \begin{cases}P_{i, t}\left(1+\eta^{p}\right) & \text { if } \Delta_{i, t}<\Delta_{i, t}^{m} \text { and } P_{i, t}<P_{t} \\ P_{i, t}\left(1-\eta^{p}\right) & \text { if } \Delta_{i, t}>\Delta_{i, t}^{M} \text { and } P_{i, t}>P_{t}\end{cases}
$$

In words: the firm revises the price up (resp: down) by $\eta^{p}$ if there is a shortage (excess) of inventories and the current price is smaller (greater) than the competitors' price.

We will now survey the specifications of this general rules adopted in some of the MABMs under scrutiny. Let's start with a small MABM. LEN posits the following quantity updating rule:

$$
Y_{i, t+1}^{*}= \begin{cases}Y_{i, t}^{*}+1 & \text { if } \Delta_{i, t}<\Delta_{i, t}^{m} \\ Y_{i, t}^{*}-1 & \text { if } \Delta_{i, t}>\Delta_{i, t}^{M}\end{cases}
$$

In words: the firm scales up production by one unit if inventories are "too low", i.e., smaller than the minimum. On the other hand, the firm scales down production if inventories are "too high", i.e., greater than the maximum. LEN assumes the firm has a one-to-one technology which uses only labor as an input: $Y_{i, t}=N_{i, t}$. Therefore, in order to scale up production by one unit, the firm has to post a vacancy. The firm scales down production by one unit by firing a worker chosen at random in the employed workforce. ${ }^{47}$

The rule (32) is a special case of (30) in which the signal coming from the relative price is ignored. Moreover the change in quantity is a discrete step of size one.

As to pricing, LEN assumes that the firm wants the actual mark up $\mu_{i, t}=\frac{P_{i, t}}{M C_{i, t}}-1$ to fall into a pre-specified interval: $\mu^{m}<\mu_{i, t}<\mu^{M}$ where $0<\mu_{m}<\mu_{M}$. The introduction of an upper and a lower bound on markups mitigates price volatility. In fact, the current price is deemed adequate if $P_{i, t}^{m}<P_{i, t}<P_{i, t}^{M}$ where $P_{i, t}^{m}=\left(1+\mu_{m}\right) M C_{i, t}$ and $P_{i, t}^{M}=\left(1+\mu_{M}\right) M C_{i, t}$ are the minimum and maximum price. LEN posits the following price updating rule:

$$
P_{i, t+1}= \begin{cases}P_{i, t}\left(1+\eta^{p}\right) & \text { if } \Delta_{i, t}<\Delta_{i, t}^{m} \text { and } P_{i, t}<P_{i, t}^{M} \\ P_{i, t}\left(1-\eta^{p}\right) & \text { if } \Delta_{i, t}>\Delta_{i, t}^{M} \text { and } P_{i, t}>P_{i, t}^{m}\end{cases}
$$

where $\eta^{p}$ is a random draw from a uniform distribution. In words: the firm raises the posted price if inventories are "too low" provided the current price is smaller than the maximum. On the other hand, the firm cuts the price if inventories are "too high" provided the current price is higher than the minimum. The size of the rate of change is stochastic.

\footnotetext{
${ }^{47}$ LEN introduces an asymmetry in quantity updating by assuming that there are firing costs: the decision to fire is implemented one month after it has been taken. The output response of the firm to an excess of inventories is therefore slower than the response to a shortage of inventories.
} 
LEN introduces inertia in price changes which may result in stickiness. In fact there is an interval of values for inventories such that the firm does not change the price. ${ }^{48}$

Expression (33) is a special case of (31) in which the signal coming from the relative price has been replaced by a an upper and lower bound on the range of the mark-up (with no reference to the prices charged by the competitors).

In AGH, before deciding to enter the market and produce, the firm conducts market research by sending out a message to people who are prospective buyers. This messages contain the "normal" posted price $P_{i, t}=(1+\mu) w_{i, t}$ where $w_{i, t}$ is the posted wage. ${ }^{49}$ The mark up $\mu$ is drawn from a uniform distribution.

As in LEN, AGH assume that the firm wants to keep a buffer stock of finished goods. Contrary to LEN, however, the desired size of inventories is unique: $\Delta_{i, t}^{*}=\delta^{*} Y_{i, t}$. AGH assume a fairly simple price updating rule:

$$
P_{i, t+1}= \begin{cases}P_{i, t}\left(1+\eta^{p}\right) & \text { if } \Delta_{i, t}<\Delta_{i, t}^{*} \\ P_{i, t}\left(1-\eta^{p}\right) & \text { if } \Delta_{i, t}>\Delta_{i, t}^{*}\end{cases}
$$

where $\eta^{p}$ is a random draw from a uniform distribution. (34) is a special case of (31) in which $\delta_{m}=\delta_{M}=\delta^{*}$ and the relative price has no role to play.

The prospective customer (household $\mathrm{h}$ ) accepts to buy if the price posted by firm $\mathrm{i}$ is smaller than the price the household previously paid - by purchasing goods at firm $\mathrm{j}$ updated by means of expected inflation, equal to the inflation target of the central bank. In symbols $P_{j, t-1}\left(1+\pi^{T}\right)>P_{i, t}$.

Transactions occur in the following way. The household visits two stores, 1 and 2. Consider one of the two, say store 1 , which has inventories $\Delta_{1, t}$. The household can place an order for $C_{1, h, t}{ }^{50}$ if a cash in advance constraint is satisfied: $P_{1, t} C_{1, h, t}<M_{h, t}$ where $M_{h, t}$ are the household's money holdings. The store offers an amount $Y_{1, t}=\min \left(C_{1, h, t}, \Delta_{1, t}\right)$. Suppose the household previously purchased goods at firm 2. The households accepts to buy from firm 1 if $P_{1, t}<P_{2, t-1}\left(1+\pi^{T}\right)$

Upon entering the market, according to AGH the firm sets a target for the labour input $N_{i, t}^{*}$ which must satisfy the requirement to produce desired output $Y_{i, t}^{*}$, given the fixed labor input (overhead costs) F and the need to move closer to desired inventories:

$$
N_{i, t}^{*}=Y_{i, t}^{*}+F+\lambda_{\Delta}\left(\delta^{*} Y_{i, t}^{*}-\Delta_{i, t}\right)
$$

where $\lambda_{\Delta}$ is the inventory adjustment speed.

In CATS, firms explore the price-quantity space around their status quo $\left(P_{i, t}, Y_{i, t}\right)$ in order to maximize profits by adapting to the market environment. For simplicity, in this overview of firms' behavior in CATS, we will assume that planned production can be always carried out: $Y_{i, t}^{*}=Y_{i, t}{ }^{51}$ Notice that desired inventories are zero in this setting, i.e. $\delta^{*}=0$.

Once transactions have been carried out, the information set of the firm $\Omega_{i, t}=\left(P_{t}, e_{i, t}\right)$ consists of two signals: the average price $P_{t}$ and the forecasting error $e_{i, t}=C_{i, t}-Y_{i, t}$.

\footnotetext{
${ }^{48}$ Moreover, LEN assumes Calvo pricing, i.e., in each period a fraction of firms cannot change their price even if the rule (33) would suggest to do so.

${ }^{49}$ Since technology is linear and productivity is normalized to one, $w_{t}$ is also the average cost.

${ }^{50}$ The amounts $C_{1, h, t}$ and $C_{2, h, t}$ are determined as shown above, in the section on "The choice of the goods to buy".

${ }^{51}$ Both in CATS/MBU and in CATS/ADG there may be constraints which prevent the attainment of desired production.
} 
These signals capture - albeit imprecisely - the distance between the firm's actual position (the status quo) and the "benchmark" i.e., a situation in which all firms charge the same price and demand equals supply: $P_{i, t}=P_{t}$ and $Y_{i, t}=C_{i, t}{ }^{52}$ There are four cases: (a) $P_{i, t}>P_{t}, e_{i, t}>0$; (b) $P_{i, t}<P_{t}, e_{i, t}>0$; (c) $P_{i, t}>P_{t}, e_{i, t}<0$; (d) $P_{i, t}<P_{t}, e_{i, t}<0$. On the basis of this information the firm decides (i) whether to change the price or the quantity in $\mathrm{t}+1$ (with respect to the status quo in $\mathrm{t}$ ), (ii) in which direction and (iii) the size of such a change.

As to quantity, the firm sets in t desired production $Y_{i, t+1}^{*}$ for $\mathrm{t}+1$ at the level of expected future demand, i.e., $Y_{i, t+1}^{*}=C_{i, t+1}^{e}$. Expectations of future demand are based on the forecasting error $e_{i, t}$. CATS/ADG assume that the firm decides to update the desired scale of activity as follows:

$$
Y_{i, t+1}^{*}=C_{i, t+1}^{e}=Y_{i, t}\left(1+\rho e_{i, t}\right) \text { if }\left\{\begin{array}{l}
e_{i, t}>0 \text { and } P_{i, t}>P_{t} \\
e_{i, t}<0 \text { and } P_{i, t}<P_{t}
\end{array}\right.
$$

where $\rho \in(0,1)$. The firm revises expected demand (and desired production) up if there is a queue of unsatisfied consumers $\left(e_{i, t}>0\right)$ and the price is higher than the average price (case (a)). The change in the scale of activity is proportional to the size of the error. If the firm's price is smaller than the average price, the firm does not adjust production even in the presence of a queue (case (b)). As we will see below, in case (b) the firm will revise the price up.

The firm revises expected demand and desired production down if $e_{i, t}=-\Delta_{i, t}<0$ and the price is lower than the average price (case $(d))$. The decrease in activity in this case is proportional to the level of involuntary inventories: $Y_{i, t+1}-Y_{i, t}=-\rho \Delta_{i, t}$. If $e_{i, t}<0$ but the price is higher than the average price, on the contrary, the firm will cut the price, keeping the quantity constant (case (c)).

Expression (36) is a special case of (30) with $\eta^{y}=\rho\left(C_{i}-Y_{i}\right)$ in case of excess demand and $\eta^{y}=-\rho \Delta_{i, t}$ in case of excess supply.

This updating rule is based implicitly on an adaptive scheme to compute expected future demand: $C_{i, t+1}^{e}=C_{i, t}^{e}+\rho\left(C_{i, t}-C_{i, t}^{e}\right)$. Recalling that $C_{i, t}^{e}=Y_{i, t}$, by iterating, it is easy to see that expected future demand is a weighted average of current and past demand with exponentially decaying weights:

$$
Y_{i, t+1}=C_{i, t+1}^{e}=\rho \sum_{s=0}^{\infty}(1-\rho)^{s} C_{i, t-s}
$$

As to the price, CATS/ADG assume the following updating rule

$$
P_{i, t+1}= \begin{cases}P_{i, t}\left(1+\eta^{p}\right) & \text { if } e_{i, t}>0 \text { and } P_{i, t}<P_{t} \\ P_{i, t}\left(1-\eta^{p}\right) & \text { if } e_{i, t}<0 \text { and } P_{i, t}>P_{t}\end{cases}
$$

where $\eta^{p} \geq 0$ is drawn from a uniform distribution. The firm revises the price up if there is a queue of unsatisfied consumers and the price is lower than the average price (case (b)): the increase of the price will mitigate excess demand for the good in question. If the firm's

\footnotetext{
${ }^{52}$ The benchmark coincides with the symmetric equilibrium in a Dixit-Stiglitz setting, in which all firms charge the same price, and each firm produces a fraction $1 / F_{c}$ of total output as shown in the section "The Dixit-Stiglitz benchmark".
} 
price is higher than the average price, in the presence of a queue the firm does not adjust the price but the quantity (case (a)).

It is easy to see that (38) is a special case of $(31)$ with $\delta^{*}=0$.

The firm revises the price down if there is an involuntary inventory and the price is higher than the average price (case (c)): the reduction of the price will boost demand (and allow to increase the market share). If the firm's price is lower than the average price, in the presence of inventories the firm does not adjust the price but the quantity (case $(d)$ ).

The mark up therefore is determined residually by the joint dynamics of the price governed by the stochastic adjustment mechanism described in (38) - and the average cost, which is determined endogenously.

It is worth noting that once the direction of the price change has been decided on the basis of the signals, the size of the price change is determined by a random draw. Contrary to the updating rule for the quantity, in the updating rule for the price the size of the adjustment does not depend on the size of the error. In CATS/MBU, there is symmetry in the updating rules, because the size of both the price and quantity adjustments are stochastic.

In this MABM an (unexpected) upward shift of the demand function (due for instance to an increase of total output, which will boost the consumption budget of each and every household) will create a queue and bring about an increase of production if the price is above the average price (case (a)); an increase of the price if the latter is below the average price (case (b)).

Financial factors seem to play no role in the price and quantity decisions of firms. This is not true. First, CATS assume there is a lower bound of the firm's price: the firm will never set the price below the average cost, which consists of operating costs (incurred to hire workers and purchase capital goods) and debt commitments. If interest payments increase, the average cost goes up and this may affect price and quantity decisions.

Second, once the scale of production has been decided, the firm goes to the bank asking for a loan to fill the financing gap (see below, section 2.4.5). But the bank may refuse to extend a loan of the requested size. In this case the firm is rationed and has to scale down production. In other words, the firm is subject to an ex post borrowing constraint.

In a couple of CATS model characterized by a network based financial accelerator, planned production is financially constrained ex ante. For instance Riccetti et al. (2013, 2016b) assume that the firm takes explicitly into account its balance sheet in deciding production. The production function is $Y_{i, t}=K_{i, t}^{\beta}$ where $K_{i, t}$ is the firm's capital stock and $\beta \in(0,1)$. The firm sets a leverage target $\lambda_{i, t}^{T} t$ where leverage is defined as the ratio of debt (bank loans) to net worth. ${ }^{53}$ Hence the target level of debt is $L_{i, t}^{T}=\lambda_{i, t}^{T} E_{i, t}$ where $E_{i, t}$ is net worth. Since $K_{i, t}=L_{i, t}^{T}+E_{i, t}$ by definition, it turns out that

$$
Y_{i, t}=\left[\left(1+\lambda_{i, t}^{T}\right) E_{i, t}\right]^{\beta}
$$

In a previous model, Delli Gatti et al. (2010) posit a financially constrained output function $Y_{i, t}=E_{i, t}^{\beta}$ which is a special case of Riccetti et al. $(2013,2016 b)$.

In EUBI, following the 'Management Science' approach, the procedure determining a firm's price and quantity is based on heuristics put forward in the literature on Operations Management and strategic pricing. A given firm (say firm i) in a given period $t_{0}$ knows the market size (total consumption expenditure) in the past $n$ periods, i.e. $C_{t_{0}-\tau}=\sum_{h=1}^{H} C_{h, t_{0}-\tau}$ for $\tau=1,2, \ldots n$. This means that the firm has a time series of aggregate consumption

\footnotetext{
${ }^{53}$ The target leverage is time varying as it is assumed to increase with expected profits.
} 
consisting of $n$ data points. The firm estimates total consumption by simple linear regression, the regressor being time: $C_{t}^{e}=\hat{C}+\hat{g} t$ where $\hat{C}$ and $\hat{g}$ are the estimated intercept and slope of the regression line. Hence the estimated current market size will be $C_{t_{0}}^{e}=\hat{C}+\hat{g} t_{0}$ and the future market size at time $t_{0}+T$ can be estimated as follows: $C_{t_{0}+T}^{e}=\hat{C}+\hat{g}\left(t_{0}+T\right)=C_{t_{0}}^{e}+\hat{g} T$. From this simple statistical exercise, the firm derives a way of updating expectations of aggregate demand for C-goods.

Then the firm adopts a market research procedure to estimate the distribution of demand $C_{i}$ for different own price levels given the price of competitors. The firm conducts a survey on a sample of consumers ${ }^{54}$ asking whether they would buy product i at $\mathrm{Z}$ different prices $P_{z}^{i}, z=1,2, \ldots Z$, given the prices of the $F^{i}$ competitors: $P_{j}, j=1,2, \ldots F_{i}$. This survey will determine a discrete distribution: each of the $\mathrm{Z}$ prices will be associated with the fraction of sampled households who would buy the product at that price, say $\pi_{z}^{i}$. In this way, the firm determines a cross-section of $Z$ data points: $\left(P_{z}^{i}, \pi_{z}^{i}\right), z=1,2, \ldots Z .^{55^{2}}$

Using the $Z$ data points the firm performs a regression of the fraction of buying households on the price according to the following specification: $\pi^{i}=\hat{b} \exp \left(-\hat{a} P^{i}\right)$ where $\hat{a}$ and $\hat{b}$ are estimated parameters. ${ }^{56}$ Current expected demand is: $C_{i, t_{0}}^{e}=\hat{b} \exp \left(-\hat{a} P^{i}\right) C_{t_{0}}^{e}$ where $C_{t_{0}}^{e}=\hat{C}+\hat{g} t_{0}$.

EUBI assume that the firm adopts an auto-regressive model to estimate the future value of the fraction. Therefore the demand curve in period $t_{0}+T$ estimated by the firm using data available up to $t_{0}$ will be: $C_{i, t_{0}+T}^{e}=\pi_{t_{0}+T}^{i} C_{t_{0}+T}^{e}$ where $C_{t_{0}+T}^{e}=\hat{C}+\hat{g}\left(t_{0}+T\right)$.

The firm then uses this estimates to determine its expected profit over a given planning horizon for each price and chooses the price which maximizes this profit. ${ }^{57}$ The pricing procedure adopted in EUBI essentially leads to mark-ups determined by the estimated price elasticity of the firm's demand and therefore is well in accordance with standard results on optimal pricing discussed in the beginning of this subsection.

Since demand is uncertain and the firm may incur "stock-out costs" because consumers will switch to competitors if the firm's inventory at a mall is empty, the firm estimates the volatility of demand (standard deviation of the demand distribution denoted by $\sigma_{C}$ ) and uses this estimate to determine a desired buffer stock. ${ }^{58}$. Production therefore must satisfy expected demand for the chosen price and generate a desired inventory:

$$
Y_{i, t_{0}+T}^{*}=C_{i, t_{0}+T}^{e}+q_{\chi} \sigma_{C}
$$

where $q_{\chi}$ is the $\chi$ quantile of the standard normal distribution and $\Delta^{*}=q_{\chi} \sigma_{C}$ are desired inventories.

In EUGE the firm estimates future demand using a linear regression on its own past sales. Given inventories, the firm sets the quantity to be produced as follows

$$
Y_{i, t+1}= \begin{cases}0 & \text { if } C_{i, t+1}^{e}<\Delta_{i, t} \\ C_{i, t+1}^{e}-\Delta_{i, t} & \text { if } C_{i, t+1}^{e}<\Delta_{i, t}\end{cases}
$$

\footnotetext{
${ }^{54}$ In the managerial literature this procedure has been labeled as simulated purchase interviews.

${ }^{55}$ The fraction can be determined also by means of the multinomial logit model.

${ }^{56}$ Hence the estimated market share of firm i when it charges the price $P_{z_{0}}^{i}$ is $\pi^{i} \mid P_{z_{0}}^{i}=\hat{b} \exp \left(-\hat{a} P_{z_{0}}^{i}\right)$.

${ }^{57}$ The level of detail reached in the model is much deeper as there are malls in different regions. Estimates should therefore be carried out at the local level.

${ }^{58}$ Problems of this kind (often referred to as "newsvendor problems") have been treated extensively in the Operations Management literature, which also provides simple decision heuristics to deal with such problems.
} 
As to pricing, EUGE assume a standard mark up pricing rule:

$$
P_{i, t}=(1+\mu) A C_{i, t}
$$

where $\mu$ is an exogenous mark up (uniform across firms) and $A C_{i, t}$ is a weighted average of the unit cost of current production and past production stocked in inventories. Costs consists of the wage bill, interest payments and the cost of capital depreciation.

In $\mathbf{K S}$, the firm plans production in $\mathrm{t}$ in such a way as to satisfy expected demand $C_{i, t+1}^{e}$ in $t+1$ and to generate the desired inventory:

$$
Y_{i, t+1}^{*}=C_{i, t+1}^{e}+\Delta_{i, t+1}^{*}
$$

The expectation of future demand $C_{i, t+1}^{e}$ is generated by past demand on the basis of an adaptive rule such as (37).

As to pricing, KS assume the following mark up pricing rule:

$$
P_{i, t}=\left(1+\mu_{i, t}\right) A C_{i, t}
$$

where $A C_{i, t}=\frac{w_{t}}{A_{j}^{\tau}}$ represents unit labor cost. $A_{j}^{\tau}$ is labour productivity due to use in production of the machine tool purchased at the j-th K-firm (see section 2.4.2).

Whereas in the MABMs reviewed so far the mark-up is either fixed or determined implicitly by the choice of the price, given the average cost, KS posit an updating rule for the mark-up:

$$
\mu_{i, t}=\mu_{i, t-1}\left(1+v \frac{f_{i, t-1}-f_{i, t-2}}{f_{i, t-2}}\right)
$$

where $v$ is a parameter and $f_{i}$ is the firm's market share. In words, the rate of change of the mark up between $\mathrm{t}-1$ and $\mathrm{t}$ is assumed to be proportional to the rate of change of the market share between $\mathrm{t}-2$ and $\mathrm{t}-1$.

The change in market share, in turn, is governed by the change in competitiveness $E_{i}$ according to a "quasi-replicator dynamics":

$$
f_{i, t}=f_{i, t-1}\left(1+\chi \frac{E_{i, t}-\bar{E}_{t}}{\bar{E}_{t}}\right)
$$

where $\bar{E}_{t}=\sum_{n=1}^{F_{c}} E_{n, t} f_{n, t-1}$ is average competitiveness of C-firms. In words: the rate of change of the market share of firm $\mathrm{i}$ is proportional to relative competitiveness $\frac{E_{i, t}-\bar{E}_{t}}{\bar{E}_{t}}$. If relative competitiveness is positive, i.e., if the $\mathrm{i}$-th firm is more competitive than the average, its share increases (and viceversa). In the evolutionary environment of KS models relative competitiveness is a selection criterion as it governs industry dynamics,i.e., expansion, contraction and extinction of firms.

Finally $E_{i, t}$ is defined as a decreasing function of the firm's price and of unfilled demand: $E_{i, t}=-\zeta_{1} P_{i, t}-\zeta_{2} U D_{i, t-1}$. In fact, customers dissatisfied with a firm which is unable to provide the goods they want to purchase will switch to competitors. Since $\bar{E}_{t}$ is a weighted average of the individual measures of competitiveness, it will be a linear function of the average price level $P_{t}$ and average unfilled demand. In the end therefore, the relative competitiveness of the $\mathrm{i}$-th firm is determined by the relative price $P_{i, t} / P_{t}$ and the relative performance in fulfilling demand. It is worth noting, for comparison, that in the the Dixit-Stiglitz benchmark, when prices are fully flexible the mark-up is determined only by 
price elasticity. In the presence of nominal rigidities, the mark up is anti-cyclical: in a Calvo setting, when the firm is unable to change the price, a demand boost will make the mark up shrink.

In JAMEL given the capital stock (see section 2.4.2), $Y_{i, t}$ is proportional to the stock of workers $N_{i, t}$. Changes in production, therefore, require proportional changes in employment obtained by hiring/firing workers. JAMEL assume that there is a maximum level of inventories the firm can hold, which is proportional to the desired level of inventories. The desired level of inventories is $\delta Y_{i, t}$. The maximum level of inventories is $\delta Y_{i, t}\left(1+\eta^{d}\right)$ where $\eta^{d} \geq 0$ is a random draw from a uniform distribution. Employment and output decisions are determined by the distance between actual inventories and the maximum level of inventories:

$$
Y_{i, t+1}^{*}= \begin{cases}Y_{i, t}^{*}\left(1+\eta^{y}\right) & \text { if } \Delta_{i, t}<\delta Y_{i, t}\left(1+\eta^{d}\right) \\ Y_{i, t}^{*}\left(1-\eta^{y}\right) & \text { if } \Delta_{i, t}>\delta Y_{i, t}\left(1+\eta^{d}\right)\end{cases}
$$

where $\eta^{y} \geq 0$ is a random draw from a uniform distribution. Pricing follows a similar updating rule:

$$
P_{i, t+1}= \begin{cases}P_{i, t}\left(1+\eta^{p}\right) & \text { if } \Delta_{i, t}<\delta Y_{i, t}\left(1+\eta^{d}\right) \\ P_{i, t}\left(1-\eta^{p}\right) & \text { if } \Delta_{i, t}>\delta Y_{i, t}\left(1+\eta^{d}\right)\end{cases}
$$

where $\eta^{p} \geq 0$ is a random draw from a uniform distribution.

In words: the firm scales up production (by posting vacancies) and increases the price if inventories are "too low"; it scales down production by firing workers and cuts the price if inventories are "too high". 5960

If desired production is equal to expected demand: $Y_{i, t+1}^{*}=C_{i, t+1}^{e}$ and involuntary inventories are a symptom of a forecasting error, the quantity updating rule above can be reinterpreted as follows. The firm revises expected demand up (stochastically) if (i) it has underestimated demand (there are no inventories) or (ii) it has overestimated demand but "not too much"; the firm revises expected demand down if it has overestimated demand by a "large" margin.

In LAGOM the firm is assumed to have a moving target for production:

$$
Y_{i, t+1}^{*}=Y_{i, t}^{*}+f\left(\pi_{i, t}, \Delta_{i, t}, K_{i, t}, C_{i, t}\right)
$$

where $f($.$) is increasing with all the arguments except inventories. The price is updated$ using a similar algorithm.

\subsubsection{Technology}

In LEN production is carried out by means of a one-to-one technology which uses only labour. In AGH capital plays a role only as collateral. each household supplies one unit of labor of a specific type which is transformed through production in one unit of goods of the same type. The technology in some of the CATS models do not use capital (e.g. CATS/MBU) or do not use labor (Delli Gatti et al. (2005)).

\footnotetext{
${ }^{59}$ In JAMEL the stochastic parameters $\eta^{d}$ and $\eta$ are correlated.

${ }^{60} \mathrm{In}$ JAMEL, as in LEN, there is inertia in pricing of the Calvo type. Each firm, in fact, is assumed to be able to set the price every $d^{p}$ periods. The fraction of firms which are able to set the price per period, therefore is $1 / d^{p}$
} 
In this section we will refer to CATS/ADG as a benchmark CATS model with capital. In CATS/ADG the i-th firm produces output $Y_{i, t}$ by means of capital $K_{i, t}$ and labor $N_{i, t}$. For simplicity ADG assume a Leontief technology. In a condition of full capacity utilization output will be

$$
Y_{i, t}=\min \left(\alpha N_{i, t}, \kappa K_{i, t}\right)
$$

where $\alpha$ and $\kappa$ are the productivities of labor and capital respectively, both exogenous, constant and uniform across firms. Assuming that labor is always abundant, $Y_{i, t}=\kappa K_{i, t}$. Hence labor requirements at full capacity utilization is $N_{i, t}=\frac{\kappa}{\alpha} K_{i, t}$, where $\kappa / \alpha$ is the reciprocal of the (given and constant) capital/labor ratio. When capacity is not fully utilized, only a fraction $\omega_{i, t} \in(0,1)$ of the capital stock will be used in production. $\omega_{i, t}$ represents the rate of capacity utilization. In this context, the marginal cost of production is constant and independent of the scale of production: $M C_{i t}=\frac{w_{t}}{\alpha}+\frac{r_{t}}{\kappa}$.

JAMEL posits the same Leontief production function but assume $\alpha=\kappa$ so that the capital/labor ratio is equal to one. Contrary to CATS/ADG, each firm has a given endowment of capital K (measured in numbers of identical machine tools) which does not depreciate and is not accumulated by means of investment. "Capital" therefore is a non reproducible and durable input which is available in fixed supply, evenly spread across firms. Hence $Y_{i, t}=\kappa K$ and labor requirement is $N_{i, t}=K$ : each machine is operated by a worker. Production takes time. Each machine needs $n$ periods to produce $\kappa$ units of output. Output is added to inventories of finished goods. As we saw above, there is a desired level of inventories (identical for all firms) $\delta \kappa K$ where $\delta$, is the desired inventories-to-output ratio.

LAGOM posit a variant of (50), namely $Y_{i, t}=\min \left(\alpha N_{i, t}, \kappa K_{i, t}, \kappa^{c} K_{i, t}^{c}\right)$ where $K_{i, t}^{c}$ is the firm's stock of circulating capital (e.g. raw materials) and $\kappa^{c}$ is the productivity of circulating capital. In the production process which takes place in one period, circulating capital is depleted completely while fixed capital and inventories of finished goods depreciate at a constant rate.

In EUBI, as well as in KS, capital goods ("machine tools") are heterogeneous since different vintages of machine tools are characterized by different productivities. We will denote with $A^{v}$ the productivity of a machine tool of vintage $v, v=1,2, \ldots, V$.

In KS K-firms develop new vintages over time by means of innovation and imitation. The probability for a K-firm to innovate or imitate $\pi_{k, t}^{z}$ with $z=$ innovate, imitate is assumed to be increasing with its $\mathrm{R} \& \mathrm{D}$ expenditure $R D_{k, t}$ :

$$
\pi_{k, t}^{z}=1-e^{-\zeta_{z} R D_{k, t}}
$$

$\pi_{k, t}^{z}$ is the parameter characterizing the Bernoulli distribution. Notice that R\&D expenditure, in turn, is proportional to past sales. Hence the probability to innovate/imitate is driven by high demand coming from C-firms, which in turn is high when households' demand for Cgoods is high. The innovating $\mathrm{K}$-firm draws a new machine embodying a technology inducing productivity $A_{k}^{v+1}$ given by:

$$
A_{k}^{v+1}=A_{k}^{v}\left(1+x_{k, t}^{A}\right),
$$

where $x_{k, t}^{A}$ is drawn from a Beta distribution characterized by parameters $\alpha_{1}$. Changes in these parameters, and therefore in the shape of the distribution, capture technological opportunities for the economy under scrutiny.

In EUBI there is complementarity between the productivity of each vintage and the skill composition of the labor force. For each vintage $v$, the actual productivity of the machine of that vintage employed in production at firm i, denoted with $A_{i}^{v}$, is constrained by the average 
skill of the workforce, denoted with $B_{i} \cdot{ }^{61}$ In symbols: $A_{i, t}^{v}=\min \left(A^{v}, B_{i, t}\right) .{ }^{62}$ If $B_{i, t}<A^{v}$, then $A_{i}^{v}=B_{i, t}<A^{v}$. In words: a machine tool with a built-in "high" productivity will under-perform if operated by a relatively unskilled workforce.

Technology to produce output with a machine tool of the v-th vintage $Y_{i, t}^{v}$ is represented by a Leontief production function, whose arguments are capital of the same vintage $K_{i, t}^{v}$ and labor employed with capital of that vintage, which we will denote with $N_{i, t}^{v}$ : $Y_{i, t}^{v}=A_{i, t}^{v} \min \left(K_{i, t}^{v}, N_{i, t}^{v}\right) \cdot{ }^{63}$ For simplicity the capital-labour ratio is 1 . The complementarity between skill of the workforce and vintage of the machine tool allows to anchor the incentive of the firm to invest in new vintages to the skill of its workforce (see Piva and Vivarelli (2009)). Summing across vintages, one gets the production function

$$
Y_{i, t}=\sum_{v=1}^{V} A_{i, t}^{v} \min \left(K_{i, t}^{v}, N_{i, t}^{v}\right)
$$

We denote with $I_{i, t}^{v}$ the purchase of a machine tool of vintage $v$. The firm will choose the vintage on the basis of the ratio of the expected future productivity of each vintage for a firm relative to the price of the machine tool of that vintage $P_{t}^{v}$. When calculating the estimated future productivity of a vintage, $\hat{A}_{i, t}^{v}$, the firm takes into account that the skills of its workforce will evolve over time and that this evolution is influenced by the vintage the workers are matched with. The probability of selecting a given vintage is then determined by means of the multinomial logit model:

$$
P[\text { firms i selects vintage } \mathrm{v}]=\frac{\exp \left(-\gamma^{v} \log \frac{\hat{A}_{i, t}^{v}}{P_{t}^{t}}\right)}{\sum_{v=1}^{V} \exp \left(-\gamma^{v} \log \frac{\hat{A}_{i, t}^{v}}{P_{t}^{v}}\right)}
$$

where $\gamma^{v}>0$. In EUBI the K-firm sets the price $P_{t}^{v}$ using a weighted average between a cost- and a value-based pricing component. To determine the value-based component the firm estimates the expected future productivity of each vintage for a firm with a labor force whose skill profile matches the average profile in the economy.

MABMs considered so far rule out substitutability among inputs (they assume indeed perfect complementarity). An exception to this rule is EUGE. The setting is similar to EUBI: there is complementarity between the quality of the capital stock and the skill composition of the labour force. The actual productivity of firm $i$ is determined by the average quality of its capital stock, $A_{i, t}$ and the average skill of the workforce $B_{i, t}$. EUGE, however, assume that technology to produce output is represented by a Cobb-Douglas production function:

$$
Y_{i, t}=\min \left[A_{i, t}, B_{i, t}\right] N_{i, t}^{\alpha} K_{i, t}^{1-\alpha}
$$

Hence inputs are substitutable. While technology in EUBI is characterized by complementarity (i) of skill and vintage and (ii) of labor and capital, in EUGE there is complementarity of skill and quality of capital, but substitutability of labour and capital.

\footnotetext{
${ }^{61} B_{i}$ is the average skill level at the firm, obtained by averaging across workers at i: $B_{i}=\frac{1}{N_{i}} \sum_{h=1}^{N_{i}} B_{h, i}$.

${ }^{62}$ Notice that $A_{v}$ is constant but $B_{i}$ is time varying, hence also $A_{i}^{v}$ is time varying.

${ }^{63} N_{i, t}^{v}=N_{i, t}-\sum_{v^{\prime}=v+1}^{V} K_{i, t}^{v^{\prime}}$
} 


\subsubsection{Demand and supply of capital goods}

In the market for K-goods (K-market for short), C-firms are buyers and K-firms are sellers.

Decisions of C-firms concerning capital accumulation have long term effects on the production possibilities of the firm. A few approaches have been proposed to describe and interpret investment decisions in a complex environment in which firms cannot perfectly anticipate future developments on goods and factor markets.

In LAGOM firms enter the market for K-goods with demand $K_{i, t}^{*}=(1 / \kappa) Y_{i, t}^{*}-K_{i, t-1}$ where $Y_{i, t}^{*}$ is the production target of the firm.

In CATS/ADG, in each period the firm decides on capacity utilization and on investment. The two decisions are carried out independently. By assumption, the firm copes with short run oscillations of demand by adjusting capacity utilization (if feasible). Investment, on the contrary, is driven by long run production requirements, as we shall see momentarily.

Let's assume that, on the basis of expected demand, the firm plans in t to produce $Y_{i, t+1}^{*}$ in t+1. In $\mathrm{t}+1$, the capital stock will be $K_{i, t+1}=K_{i, t}\left(1-\delta^{r} \omega_{i, t}\right)+I_{i, t}$ where $\delta^{r}$ is the depreciation rate, $\omega_{i, t}$ is the utilization rate and $I_{i, t}$ is investment decided in t. There are two scenarios.

If the capital stock is "large" - i.e., $Y_{i, t+1}^{*}<\kappa K_{i, t+1}$ - the firm will reach the desired scale of activity by setting capacity utilization at the required level $\omega_{i, t+1}^{*}=Y_{i, t+1}^{*} / \kappa K_{i, t+1}$ with $\omega_{i, t+1}^{*}<1$.

If, on the contrary, the capital stock is "insufficient" - i.e., $Y_{i, t+1}^{*}>\kappa K_{i, t+1}$ - the firm will use machinery and equipment at full capacity but will produce less than desired because its scale of activity is constrained by the available capital stock $K_{i, t+1}$.

Investment decisions in $t$ are based on the stock of capital the firm has used on average until t-1, computed by means of the following adaptive rule: $\hat{K}_{i, t-1}=\xi \hat{K}_{i, t-2}+(1-\xi) \omega_{i, t-1} K_{i, t-1}$ where $\xi \in(0,1)$. By iterating, it is easy to see that $\hat{K}_{i, t-1}$ is a weighted average of past utilized capital with exponentially decaying weights:

$$
\hat{K}_{i, t}=(1-\xi) \sum_{s=0}^{\infty} \xi^{s} \omega_{i, t-s-1} K_{i, t-s-1}
$$

The history of capacity utilization in the past, therefore, will influence the decision to purchase new capital goods in $\mathrm{t}$ and this decision, in turn, will impact on capacity in the future.

The firm is assumed to have a target long run capacity utilization rate $\omega^{T}$. Therefore the capital desired at the investing stage $\mathrm{t}$ for $\mathrm{t}+1$ is $K_{i, t+1}^{*}=\hat{K}_{i, t-1} / \omega^{T}$. Investment to replace worn out capital is $\delta^{r} \hat{K}_{i, t-1}$. Hence total investment will be $I_{i, t}=\left(\frac{1}{\omega^{T}}+\delta^{r}\right) \hat{K}_{i, t-1}-K_{i, t}$ where $K_{i, t}=\left(1-\delta^{r} \omega_{i, t-1}\right) K_{i, t-1}+I_{i, t-1}$.

CATS/ADG assume moreover that there are adjustment costs of the capital stock such that only a fraction of $\mathrm{C}$-firms is capable to purchase new capital goods in each period. Hence the firm has to take into account the fact that investment can be carried out only infrequently. Durability and stickiness imply that the capital stock available for production at the beginning of period $t+1$ keeps memory of the entire history of the capital stock in the past.

The demand side of the market for K-goods if populated by C-firms. The i-th C-firm plans to purchase K-goods in the amount $I_{i, t}$. 
Consider now the supply side, which is populated by $F_{k} \mathrm{~K}$-firms. Each K-firm has market power due to transaction costs (e.g. incompleteness of the information set) which force Cfirms to visit only a subset of the population of K-firms. The i-th C-firm, in fact, does not interact globally with all the producers of machine tools but randomly selects $Z_{k} \mathrm{~K}$-firms, sorts them by selling price and demand capital goods starting from the firm charging the lowest price. K-firms with lower prices, therefore, will get, in principle, higher demand.

At the beginning of time $t$ the $\mathrm{k}$-th producer of capital goods $\left(k=1,2, \ldots, F_{k}\right)$ sets the status quo $\left(P_{k, t}, Y_{k, t}\right)$.

Once production has been carried out ${ }^{64}$ and search and matching has taken place the k-th firm observes the amount of K-goods actually sold $Q_{k, t}=\min \left(Y_{k, t}, J_{k, t}\right)$ where $J_{k, t}$ is actual demand, coming from C-firms which placed orders at firm k.

If production is greater than demand, the firm will pile up an involuntary inventory of unsold goods. Since K-goods are storable, inventories accumulated in t can be used to satisfy demand in the future. If, on the contrary, demand is greater than production, the firm can satisfy excess demand by depleting inventories carried oven from the past.

K-firms receive two signals, like C-firms: the price charged by competitors and the change of inventories or the presence of queues. On the basis of these signals the firm changes the price or the quantity. Price and quantity updating rules for K-firms are similar to the ones adopted by C-firms, the major difference being the assumption that machine tools can be stored in warehouses in $\mathrm{t}$ and sold to $\mathrm{C}$-firms in the future.

In EUBI the i-th C-firm purchases new machine tools if the current capital stock is not sufficient to produce planned output. EUBI define potential output as production the firm can carry out in t using at full capacity the capital stock inherited from the past in the absence of investment, i.e., $\hat{Y}_{i, t}=\sum_{v \in V}\left(1-\delta^{r}\right) A_{i, t}^{v} K_{i, t-1}^{v}$. The investment decision is the outcome of a comparison between potential output and desired output $Y_{i, t}^{*}$.

If the capital stock inherited from the past is "large": $\hat{Y}_{i, t}>Y_{i, t}^{*}$, the firm can produce desired output by adjusting capacity utilization and there is no investment. If desired production is greater than potential output, so that the capital stock inherited from the past is "insufficient", the firm expands the capital stock and investment is positive. The size of the investment depends on the vintage $v$ chosen by the firm for the investment in that period $($ see $(54))$ :

$$
I_{i, t}^{v *}= \begin{cases}0 & \text { if } Y_{i, t}^{*} \leq \hat{Y}_{i, t} \\ \left(Y_{i, t}^{*}-\hat{Y}_{i, t}\right) / A_{i, t}^{v} & \text { if } Y_{i, t}^{*}>\hat{Y}_{i, t}\end{cases}
$$

Due to the flexibility in production due to input substitutability, in EUGE the capitallabor ratio is a function of the relative costs of the inputs:

$$
\frac{K_{i, t}}{N_{i, t}}=\frac{(1-\alpha) \hat{w}_{i, t}}{\alpha c_{i, t}^{k}}
$$

where $\hat{w}_{i, t}$ is the real wage and $c_{i, t}^{k}$ is the user cost of capital, a function of the real interest rate, the depreciation rate and the price of $\mathrm{K}$-goods.

Plugging (58) in (55) and rearranging we get the demand for capital:

$$
K_{i, t}^{*}=\left(\frac{(1-\alpha) \hat{w}_{i, t}}{\alpha c_{i, t}^{k}}\right)^{\alpha} \frac{Y_{i, t}^{*}}{A_{i, t}}
$$

\footnotetext{
${ }^{64}$ For simplicity, K-firms produce machine tools using only labor.
} 
If the current capital stock in the absence of investment is smaller than desired capital, the firm adjusts capacity utilization. If the opposite holds true the firm purchases new capital goods to bring capital up to the desired size.

K-firms produce and sell tailor-made machine tools to each purchasing C-firm, using only energy and raw materials, imported from abroad. The price of machine tools is a mark up on energy prices. Since they follow a job production process, K-firms don't have inventories. By construction, moreover, they don't have financing needs.

In KS, as in CATS/ADG, for simplicity, K-firms produce machine tools of different vintages using only labor. K-firms send "brochures" to selected C-firms advertising the price and the quality of the machine tools they produce. C-firms compare brochures and then choose the machines with the lowest price and unit cost of production and send their orders to the corresponding machine manufacturer. Gross investment of each C-firm is the sum of expansion and replacement investment, where, as above, expansion happens if the desired level of production is higher than what can be produced with the current capital stock.

\subsubsection{The demand for labor}

In LEN, AGH and JAMEL quantity decisions translate immediately into hiring/firing decisions. In LEN, thanks to the one-to-one technology, vacancies are derived straightforwardly from the quantity adjusting rule. If inventories are "too low" the firm will post a new vacancy to increase production. In symbols: $V_{i, t+1}^{*}=+1$ if $\Delta_{i, t}<\delta_{m} Y_{i, t}$. If, on the contrary, inventories are "too high", the firm will fire a worker in order to reduce production: $N_{i, t+1}=N_{i, t}-1$ if $\Delta_{i, t}>\delta_{M} Y_{i, t}$.

The firm adjusts the posted wage according to the following rule:

$$
w_{i, t+1}= \begin{cases}w_{i, t}\left(1+\eta^{w}\right) & \text { if } \Delta_{i, t} \leq \delta_{0} Y_{i, t} \\ w_{i, t}\left(1-\eta^{w}\right) & \text { if } \Delta_{i, t}>\delta_{1} Y_{i, t}\end{cases}
$$

where $\eta^{w} \geq 0$ is drawn from uniform distribution.

In JAMEL maximum employment per firm coincides with the capital stock. Hence we can define capacity utilization $\omega_{i, t}=N_{i, t} / K$. JAMEL assume also that there is a target or desired utilization rate $\omega^{T}$. The firm adjusts the posted wage according to the following rule:

$$
w_{i, t+1}= \begin{cases}w_{i, t}\left(1+\eta^{w}\right) & \text { if } \omega_{i, t}-\omega^{T}>\tilde{\omega} \\ w_{i, t}\left(1-\eta^{w}\right) & \text { if } \omega_{i, t}-\omega^{T}<\tilde{\omega}\end{cases}
$$

where $\eta^{w} \geq 0$ is drawn from a uniform distribution and $\tilde{\omega}$ is a stochastic cut-off value.

In AGH the firm conducts market research before deciding to enter the market and produce by sending out messages to people who are prospective employees. This message contains the posted wage. AGH assume that the wage is set by the firm as

$$
w_{i, t}=\nu_{i, t} w_{i, t-1}\left(1+\pi^{T}\right)
$$

where $\nu_{i, t}$ is a function of the ratio of desired employment to the workforce $\nu_{i, t}=f\left(N_{i, t}^{*} / N_{i, t-1}\right)$ and $\pi^{T}$ is expected inflation, which is equal to the (time invariant) inflation target of the central bank. AGH assume that the central bank's target for inflation is the anchor agents used in expectation formation. 
In most MABMs with capital, the demand for labor is determined by planned production and the size of the capital stock, with limited substitutability between labor and capital. Consider, for instance, CATS/ADG. Let's assume that, on the basis of expected demand, the C-firm plans to produce $Y_{i, t+1}^{*}$ in $t+1$. Suppose that technology is represented by a Leontief production function (see above). If the capital stock is "large", then $\omega_{i, t+1}^{*}<1$. Hence the firm could reach the desired scale of activity by setting capacity utilization at the required level and adjust desired employment accordingly. In symbols $N_{i, t+1}^{*}=(1 / \alpha) Y_{i, t+1}^{*}=$ $\omega_{i, t+1}(\kappa / \alpha) K_{i, t+1}$.

If, on the contrary, the capital stock is "insufficient" the firm will use the stock of capital at full capacity $\left(\omega_{i, t+1}=1\right)$ and $Y_{i, t+1}=\kappa K_{i, t+1}$ so that desired employment in this case will be $N_{i, t+1}^{*}=(\kappa / \alpha) K_{i, t+1}$. Hence planned employment will be

$$
N_{i, t+1}^{*}=\omega_{i, t+1} \frac{\kappa}{\alpha} K_{i, t+1}
$$

where $0<\omega_{i, t+1} \leq 1$ and $K_{i, t+1}=\left(1-\delta^{r} \omega_{i, t}\right) K_{i, t}+I_{i, t}$

In EUBI and EUGE, if the capital stock is "too low" in a given period, say $\mathrm{t}+1$, investment can be carried out in the same period to overcome bottlenecks in production, also in the short run (see previous section) so that $K_{i, t+1}=\left(1-\delta^{r}\right) K_{i, t}+I_{i, t+1}$.

In EUBI, with a Leontief technology, the demand for labor is

$$
N_{i, t+1}^{*}=\frac{\kappa}{\alpha} K_{i, t+1}
$$

The same equation defines the demand for labor in LAGOM.

In EUGE, with a Cobb-Douglas technology, the demand for labor is

$$
N_{i, t+1}^{*}=\frac{\alpha c_{i, t}^{k}}{(1-\alpha) \hat{w}_{i, t}} K_{i, t+1}
$$

Given planned employment $\hat{N}_{i, t+1}$ and actual employment, $N_{i, t}$, at the beginning of period $t+1$ the firm posts vacancies as follows:

$$
V_{i, t+1}^{*}=\max \left(N_{i, t+1}^{*}-N_{i, t}, 0\right)
$$

Suppose $N_{i, t+1}^{*}>N_{i, t}$. In CATS/ADG a vacancy is filled only if the firm is visited by an unemployed worker. The probability of filling the vacancy depends on the posted wage and on the number of unemployed workers visiting the firm. Thus actual employment $N_{i, t+1}$ may be smaller than desired employment $N_{i, t+1}^{*}$, the difference between the two being unfilled vacancies. In this case the planned scale of activity will not be reached.

Suppose now $N_{i, t+1}^{*}<N_{i, t}$, i.e the number of actual workers exceeds the level of desired employment. In this scenario the firm fires $n_{i, t+1}=N_{i, t}-N_{i, t+1}^{*}$ workers selected at random. Similarly, in EUBI the actual workforce can differ from planned employment due to search frictions and differences in wages offered by different firms If employment is too high, there is random firing.

Summing up, the firm enjoys full flexibility in adjusting production when production must be scaled down (first scenario) and when output should be increased but "not too much" (i.e., when capacity utilization can be adjusted adequately). When the desired increase in output is sizable the firm hits a capacity constraint. 
Actual production may be smaller than desired if the firm does not succeed in achieving the "appropriate" level of capital but also if the firm does not succeed in achieving the "appropriate" level of employment (for instance because of unfilled vacancies) and/or she does not get enough credit.

Consider now K-firms. In CATS/ADG the k-th firm produces output $Y_{k, t}$ by means of a linear technology which uses only labor: $Y_{k, t}=\alpha N_{k, t}$ where $\alpha$ is the productivity of labor, exogenous, constant and uniform across firms. Desired employment will be $N_{i, t+1}^{*}=Y_{i, t+1}^{*} / \alpha$. The firm posts vacancies: $V_{k, t+1}=\max \left(N_{k, t+1}^{*}-N_{k, t}, 0\right)$. These vacancies add to those posted by C-firms.

On the labor market C-firms and K-firms are competing for labor services: labor is a homogeneous input. Unemployed workers search for a job visiting $Z_{e}$ firms (out of a population of $\mathrm{F}$ firms) irrespective of the type of goods they produce. In other words, the unemployed worker is indifferent between finding a job at a C-firm or a K-firm.

\subsubsection{The demand for credit}

In most MABMs, the demand for credit comes from the corporate sector only and is determined by the firms' financing needs. Consider a generic firm, say $f$ (where $f=i, k$ ), with operating costs $X_{f, t}$ and internal funds $M_{f, t-1}$. If the latter is greater than the former, the firm can finance costs by means of internal funds only. If, on the contrary, costs are greater than internal funds, the firm has a financing gap

$$
F_{f, t}=\max \left(X_{f, t}-M_{f, t-1}, 0\right)
$$

which must be filled by means of a bank loan. The demand for credit, therefore, coincides with the financing gap. ${ }^{65}$

Consider a C-firm. If technology requires only labour, operating costs coincide with the wage bill. Hence the financing gap is: $F_{i, t}=\max \left(w_{i, t} N_{i, t}-M_{i, t-1}, 0\right)$. This is essentially the demand for credit in AGH, LEN and JAMEL. ${ }^{66}$

If the firm employs labour and capital, the firm has to finance also the purchase of new K-goods. For example, in CATS/ADG the expenditure on capital goods is $P_{t-1}^{K} I_{i, t}$ where $P^{K}$ represents the price index of K-goods. ${ }^{67}$

Consider now a K-firm. If technology requires only labor, the financing gap is $F_{k, t}=$ $\max \left(w_{k, t} N_{k, t}-M_{k, t-1}, 0\right)$.

We can posit, therefore, the following general specification of the demand for credit:

$$
F_{f, t}=\max \left(w_{f, t} N_{f, t}+\mathbf{1}_{\mathbf{k}} P_{t-1}^{K} I_{f, t}-M_{f, t-1}, 0\right)
$$

where $\mathbf{1}_{\mathbf{k}}$ is an indicator function which assumes value 1 if the firm employs capital and labour and 0 if it employs only labour.

\footnotetext{
${ }^{65}$ In EUGE firms can obtain external finance also in other ways.

${ }^{66}$ In AGH the firm has a financing need already at entry because there are set up costs that should be financed.

${ }^{67}$ In CATS/ADG, at the moment of deciding investment in $\mathrm{t}$ the $\mathrm{C}$-firm does not have sufficient information about the prices charged by K-firms (a decision also taken in $\mathrm{t}$ ). Therefore, in order to evaluate the financing gap, the firm uses the mean of the prices determined in $\mathrm{t}-1$.
} 


\subsection{The Bank}

In most MABMs C-firms demand labor and K-goods in order to produce and sell C-goods to households.

\subsubsection{Credit risk and the interest rate}

The benchmark: financial frictions In the most recent literature, a special and crucial role for financial factors in macroeconomic performance stems from the assumption of "financial frictions", i.e. imperfections in the transmission of funds from surplus units (lenders) to deficit units (borrowers). ${ }^{68}$ There are essentially three types of financial friction.

\section{Costly state verification}

Building on Bernanke and Gertler (1989) and Bernanke and Gertler (1990), Bernanke et al. (1999) (BGG herafter), develop a NK-DSGE model in which a financial friction arises from ex post asymmetric information between borrowers ("entrepreneurs" 69) and lenders (banks) in the presence of costly state verification à la Townsend. Information on the return of investment becomes asymmetric ex post, i.e., after the investment has been carried out. While the entrepreneur can observe the return on investment at zero cost, the bank can ascertain the "true return" only incurring a monitoring cost.

To be more specific, consider the balance sheet of the representative entrepreneur

$$
q_{t} K_{t}=L_{t}+E_{t}
$$

where $K$ is physical capital (a durable asset), $q$ is the market value of the firm's assets $L$ is debt towards banks and $E$ is net worth. Hence $\lambda_{t}:=q_{t} K_{t} / E_{t}$ is leverage. ${ }^{70}$ The optimal financial contract between the entrepreneur and the bank in such a setting yields a risk premium - i.e. a wedge between the interest rate on loans and the risk free interest rate which BGG label the "External Finance Premium" (EFP): $E F P_{t}=R_{L} / R{ }^{71}$. They show that the EFP is increasing with the borrower's leverage: $E F P_{t}=\phi\left(\lambda_{t}\right)$ with $\phi^{\prime}>0$. In the end, therefore

$$
R_{t}^{L}=R_{t} \phi\left(\lambda_{t}\right)
$$

In BGG the bank sets (optimally) the interest rate on loans (i.e. the price of credit) as a mark up over the risk free interest rate, the mark up being increasing with leverage. At that price all the credit demanded is extended. The quantity of credit is demand-driven.

\section{Costly enforcement}

\footnotetext{
${ }^{68}$ Lenders are generally banks, borrowers are generally firms. These frictions generate a "financial accelerator". Hence financial instability is interpreted as the amplification of the macroeconomic effects of a shock in a linear impulse-propagation framework.

${ }^{69}$ In Bernanke et al. (1999) entrepreneurs are firms which purchase K-goods to produce wholesale, homogeneous goods. These goods are sold to "retailers" who differentiate them and sell final goods to households and K-firms.

${ }^{70} \mathrm{~A}$ more encompassing balance sheet: $M_{t}+q_{t} K_{t}=L_{t}+E_{t}$ where $\mathrm{M}$ is the firm"s liquidity. If the firm produces using only labor, durable assets do not show up in the balance sheet. Liquidity, therefore, of both internal and external origin, is used to finance the wage bill only: $\mathrm{M}=\mathrm{wN}$ and $\mathrm{L}=\mathrm{M}-\mathrm{E}=\mathrm{wN}-\mathrm{E}$.

${ }^{71}$ To be precise, the EFP is $E F P=R^{K} / R$ and The interest rate on loans $R_{L}$ is somehow proportional to the rate of return on capital.
} 
Kiyotaki and Moore (1997) (KM hereafter) assume that the human capital of the producer/borrower is critical to production and "inalienable" à la Hart and Moore. In this setting there is a financial friction due to costly enforcement of the debt contract: if the borrower "takes the money and runs" the lender cannot replace him with a new producer: producers are not substitutable. In a default scenario the lender is bound to experience a loss. Therefore she will design the debt contract in such a way as to minimize this loss.

The debt contract specifies the interest rate $R^{L}$, the size of the loan $(L)$, maturity (one period) and the provision of collateral $(K)$ on the part of the borrower. Due to costly enforcement, the borrower is subject to a financing constraint:

$$
L_{t}=\frac{q_{t+1} K_{t}}{R^{L}}
$$

where $L_{t}$ is the loan the lender is willing to extend, $K_{t}$ is a durable asset pledged by the borrower as collateral, which we will identify with "capital", ${ }^{72} q_{t+1}$ is the price of capital at maturity, $R^{L}$ is the interest rate on loans, uniform across agents, given and constant. The financing constraint states that the lender extends a loan in t of a size such that debt commitments (interest and principal) $R^{L} L_{t}$ at maturity, i.e., in $\mathrm{t}+1$, is equal to the market value of collateral $q_{t+1} K_{t}$. Thanks to the financing constraint, if the borrower defaults, at maturity the lender can repossess capital and sell it, earning sales proceeds $q_{t+1} K_{t}$ exactly equal to the borrower's debt commitments. The lender's loss in case of default therefore is reduced to zero.

Plugging (71) into (69) and rearranging we get $K_{t}=\lambda_{t} E_{t}$ where $\lambda_{t}=\left(q_{t}-\frac{q_{t+1}}{R^{L}}\right)^{-1}$ is leverage. ${ }^{73}$ Hence, leverage is increasing with $q_{t+1}$ but decreasing with $q_{t}$ and $R^{L}$.

In KM the quantity of credit is set by the lender as a proportion of the market value of collateral. The price of credit is exogenous.

If the production function is $Y_{t}=Y\left(K_{t}\right)$, then the scale of activity of the producer will be constrained by his wealth (net worth):

$$
Y_{t}=Y\left(\lambda_{t} E_{t}\right)
$$

Costly bankruptcy Greenwald and Stiglitz (1993) (GS hereafter) shift the burden of bankruptcy costs from the lender to the borrower. For GS it is the borrower who incurs the (pecuniary and reputational) costs due to default. These costs are the source of a financial friction which shows up as a wedge between the interest rate on loans as perceived by the borrower and the cost of credit set by the lender.

Consider a generic firm which gets a loan $L_{t}$ from a bank at the rate $R$. Debt commitments therefore are $R L_{t}$. The firm incurs bankruptcy costs $C B_{t}$ with probability $\pi_{t}^{d}$. GS assume that the costs of bankruptcy are increasing with size: $C B_{t}=c^{d} Y_{t}$ where $c^{d}$ is the (given) cost of default per unit produced. Moreover, they show that when revenues are uncertain, the probability of default is increasing with leverage: $\pi_{t}^{d}=\pi\left(\lambda_{t}\right), \pi^{\prime}>0$. Hence total costs

\footnotetext{
${ }^{72}$ Kiyotaki and Moore (1997) interpret $\mathrm{K}$ as land, which is non reproducible and available in fixed supply. Capital is durable (as land) but also reproducible and in variable supply. However, both capital and land can be used as collateralizable wealth.

${ }^{73}$ Leverage is the reciprocal of the "downpayment" in Kiyotaki and Moore (1997). The down-payment is the ratio of equity (internal funds) to the market value of the durable asset the agent wants to hold. For each unit of a durable asset the agent wants to hold (land, capital), the down-payment defines the amount of internal funds the agent has to provide. The size of the loan per unit of asset is provided by the lender as a loan. Hence, the complement to unity of the down-payment is the loan-to-value ratio.
} 
(the sum of debt commitments and expected bankruptcy costs) are $\left[R+c^{d} y^{d} \pi_{t}^{d}\right] L_{t}$ where $y^{d}$ is output per unit of credit. The actual cost of credit (i.e., the interest rate on loans for the borrower) therefore is

$$
R_{t}^{L}=R+c^{d} y^{d} \pi\left(\lambda_{t}\right)
$$

The actual cost of credit is the interest rate set by the bank augmented by a risk premium $c^{d} y^{d} \pi\left(\lambda_{t}\right)$ borne by the borrower. It is the borrower who perceives the risk of default. In Bernanke et al. (1999), the risk of default is borne by the lender. ${ }^{74}$

The Agent Based approach to interest rate setting In AGH, there is a bank in each sector of the economy, loosely defined as a subset of the number of goods. The bank accepts deposits from people in its sector and extend loans to firm owners. Loans are collateralized by inventories and fixed capital (which plays only the role of collateral, it is not an input). If a firm is unable to reimburse debt the bank can repossess all the collateral and the wealth of the firm owner (money and deposits) ("full recourse"). The interest rate on deposits is equal to the interest rate set by the central bank. In many ABMs the interest rate set by the central bank is either fixed or follows a Taylor rule. For instance, in AGH, LAGOM, $\mathrm{KS}$ and EUGE, the interest rate is assumed to follow a standard Taylor rule in the flexible infation targeting regime

$$
r=\max \left[r^{T}+\gamma_{\pi}\left(\pi-\pi^{T}\right)+\gamma_{y}\left(y-y^{T}\right), 0\right]
$$

where $r^{T}$ is the target for the "long run" interest rate ${ }^{75}$ and $\pi^{T}, y^{T}$ are the targets for inflation and output respectively. While $\pi^{T}$ is fixed, in AGH the target for potential output and the target for the long run interest rate are updated. The interest rate on loans is a fixed markup on this rate, uniform across firms.

In CATS/ADG firms demand credit according to their production plans and the resulting financing gaps (as shown above). For simplicity, there is only one bank, which has to decide both the price (interest rate) and the quantity of loans to extend to each firm on the basis of the assessment of the firm's financial fragility proxied by leverage.

For each borrowing firm $f$ in each period the bank can compute the leverage ratio $\lambda_{f, t}{ }^{76}$

The bank will estimate the relationship between the individual bankruptcy probability $\pi_{f, t}^{d}$ and the individual leverage $\lambda_{f, t}: \pi_{f, t}^{d}=\pi\left(\lambda_{f, t}\right)$. The estimated default probability is an increasing function of leverage.

For simplicity, the bank is assumed to be risk neutral: she will lend to a risky firm inasmuch as the return on lending to that firm is not smaller than the return on investing in an alternative risk free asset which measures the opportunity cost of lending. ${ }^{77}$

Once all the arbitrage opportunities have been exploited, the following equality will hold true: $R_{f, t}\left(1-\pi_{f, t}^{d}\right)=R$. Hence

$$
R_{f, t}=\frac{R}{1-\pi\left(\lambda_{f, t}\right)}
$$

\footnotetext{
${ }^{74}$ If operating costs are $X_{t}$ and credit fills the financing gap, then total costs are $\left[R+c^{d} y^{d} \pi\left(\lambda_{t}\right)\right]\left(X_{t}-E_{t}\right)$. Suppose that, with DRS, $X_{t}=\frac{x}{2} Y_{t}^{2}$ Profits are: $\Pi_{t}=Y_{t}-\left[R+c^{d} y^{d} \pi\left(\lambda_{t}\right)\right]\left(\frac{x}{2} Y_{t}^{2}-E_{t}\right)$ The optimum is $Y_{t}=\left(\left[R+c^{d} y^{d} \pi\left(\lambda_{t}\right)\right] x\right)^{-1}$ Here output is decreasing with leverage, because, otbe, an increase of leverage increases marginal cost. In KM an increase of leverage boosts output because makes more credit available per unit of equity and therefore more capital.

${ }^{75}$ Natural rate

${ }^{76}$ To be precise, CATS/ADG use a definition of leverage $\lambda^{\prime}=\frac{L}{E}$ which is a linear transformation of the one used in this paper and elsewhere in this literature: $\lambda=\frac{L+E}{E}$. In fact $\lambda=1+\lambda^{\prime}$.

${ }^{77}$ This is the definition of the participation constraint for the lender.
} 
From (75) follows that the interest rate charged by the bank to the $\mathrm{f}$-th firm is increasing with the risk free rate and with the firm's leverage. This is a reduced for a BGG type of friction. If the firm goes bankrupt, the equity of the bank will decrease.

Also in JAMEL there is only one bank, which accommodates the demand for credit at a fixed interest rate $r$ and maturity $d_{L}$, (uniform across borrowing firms). If a firm - say firm $\mathrm{i}$ - enters a period of financial distress and becomes unable to validate debt commitments as scheduled, the loan becomes "doubtful" so that the bank raises the interest rate to $r_{i}>r$ and extends maturity to $d_{i}>d_{L}$.

In EUBI and EUGE the bank assesses the probability of default as follows:

$$
\pi_{f, t}^{d}=1-e^{-\lambda_{f, t}}
$$

The interest rate charged by the bank to the firm therefore is given by:

$$
R_{f, t}=R+k \pi_{f, t}^{d}
$$

where $k$ is a parameter.

\subsubsection{Supply of loans}

In most MABMs, the bank is subject to a regulatory prudential constraint consisting of a minimum "capital requirement" of the Basel type: the bank must have "capital" (net worth) $E_{b, t}$ at least equal to a given fraction of risky assets $R A_{b, t}$. Defining the bank's leverage as $\lambda_{b, t}=R A_{b, t} / E_{b, t}$, the regulatory constraint can be recast in terms of maximum leverage: the bank can hold illiquid (risky) assets up to a maximum equal to a multiple $\lambda_{b}^{M}$ of net worth. In symbols: $R A_{b, t} \leq \lambda_{b}^{M} E_{b, t}$ where $\lambda_{b}^{M}$ is the bank's upper bound on bank's leverage set by the regulator.

In AGH risky assets are $R A_{b, t}=L_{b, t}+K_{b, t}$ where $K_{b, t}$ is the level of seized collateral. The probability for the bank to approve new loan applications is

$$
\pi_{b, t}^{L}=\min \left[k_{b}\left(\frac{\lambda_{b}^{M} E_{b, t}}{L_{b, t}+K_{b, t}}-1\right), 1\right]
$$

The bank will extend loans for sure if $L_{b, t}+K_{b, t} \leq \frac{k_{b}}{1+k_{b}} \lambda_{b}^{M} E_{b, t}$ i.e., if $\lambda_{b, t}<\lambda_{b}^{M} \frac{k_{b}}{1+k_{b}}$. When this threshold is achieved, the probability of approving a new loan becomes less than one. It becomes zero when the maximum regulatory requirement is reached, i.e., $\lambda_{b, t}=\lambda_{b}^{M}$.

In CATS/ADG and KS risky assets coincide with loans. Hence the regulatory constraint boils down to $L_{b, t} \leq \lambda_{b}^{M} E_{b, t}$. Suppose the constraint is binding. Hence the maximum amount of credit the bank can extend to all firms/borrowers is constrained by the availability of internal finance:

$$
L_{b, t}=\lambda_{b}^{M} E_{b, t}
$$

At this point the bank has to allocate credit to each firm. CATS/ADG assume that bank b opens a credit line $\Lambda_{f, t}$ to firm f up to a maximum represented by a fraction $\psi_{f, t}$ of total credit $L_{b, t}$. This fraction is decreasing with the firm's leverage: $\psi_{f, t}=\psi\left(\lambda_{f, t}\right)$ with $\psi^{\prime}<0$. In symbols: $\Lambda_{f, t}=\psi\left(\lambda_{f, t}\right) L_{b, t}$. KS posit a similar procedure: borrowing firms are ordered in terms of financial fragility, captured by the ratio of net worth to past sales.

If the loan demanded by the firm (i.e. the financing gap $F_{f, t}$ ) is smaller than the maximum loan the bank is willing to extend (i.e. $\Lambda_{f, t}$ ), then the bank will accommodate the demand 
for credit: $L_{f, t}=F_{f, t}$; if, on the contrary, the financing gap is greater than the credit line the bank is willing to extend, the bank will limit credit to the borrower: $L_{f, t}=\Lambda_{f, t}$. In the end therefore:

$$
L_{f, t}=\min \left(F_{f, t}, \Lambda_{f, t}\right)
$$

From the inequality above, given the definition of financing gap follows that an increase of the net worth of the bank and/or of the borrowing firm - so that the firm's leverage and the estimated probability of bankruptcy go down - relaxes the constraint represented by the maximum admissible loan supply and makes credit rationing less likely.

In EUBI and EUGE, the bank computes the budget available to fund firms as follows:

$$
L_{b, t}=\lambda_{b}^{M} E_{b, t}-A_{b, t}
$$

where $A_{b, t}$ is the sum of risk weighted assets in the bank's portfolio. The bank has to decide now how much credit to extend to each firm. In EUBI the firm's financing gap is funded completely if the credit risk exposure remains below the budget, while the firm is fully rationed if the loan exceeds this limit.

\section{Comparison of Existing Agent-based Macroeconomic Models}

Whereas in the previous section we have discussed in some detail several key issues in designing MABMs, the purpose of this section is to provide a more general survey and comparison over several agent-based macroeconomic models, which have been developed during these years. We discuss the variance of the model structure and the behavioral assumptions across the different approaches and examine in how far an established 'core' of modeling assumptions has emerged for agent-based macroeconomics. The basis for this discussion are Tables A1 and A2 in Appendix A, which provide a systematic overview of main properties of the eight agent-based macroeconomic models that have already been the basis for the discussion in Section 2.

The different models covered here have been designed and implemented for various research purposes in macroeconomics and with different agendas in mind.

- MABMs in which "financial factors" play a major role in business fluctuations: AGH, CATS, EUGE;

- MABMs in which capital accumulation, embodied technical progress and skill dynamics generate growth, fluctuations and inequality dynamics: KS, EUBI

- MABMs with focus on policy analyses, in particular on certain measures in fiscal, monetary or climate policy: KS, EUBI, EUGE, LAGOM

- MABMs which capture the effects of learning of agents, social influence and opinion dynamics: JAMEL

- MABMs with minimal complexity that are able to reproduce key stylized facts like endogenous business cycles, Phillips and Beveridge curves: LEN 
The presentation of the models in Tables A1 and A2, as well as our discussion of the similarities and differences between the different models, is structured along eight broad categories covering different aspects of the setup of the models and their main output. As observed in Section 2, in terms of the general model structures, we observe that almost all share the same set of types of agents (households, C-firms, K-firms, banks, policy makers) and considered markets (consumption goods, capital, labor, credit, stocks) except for LEN, which does not include physical capital, credit and banks in the model, and AGH, which also abstracts from physical capital accumulation. Recent literature on agent-based macroeconomics has stressed the importance of the concept of stock-flow consistency for models of this type (e.g. Caiani et al. (2016a)). Stock-flow consistency entails that the model is closed in a sense that for any transaction the (real and financial) flows of all involved agents sum up to zero, such that there are no (hidden) inflows or outflows. This can be most easily ensured if the model encompasses explicit balance sheets for all agents, but only a fraction of the MABMs have these properties, and therefore it is not straight forward to verify this property if it is not directly reported by the authors.

In terms of other general properties, it becomes clear that in most MABMs expectations are mainly considered with respect to the future development of firm demand, whereas expectation formation of households about future income or economic developments is often not considered. The employed expectation rules are in most MABMs naive or linear forecasts. An exception in this respect is JAMEL, which models the dynamics of household expectations driven by evolving sentiments in the population. In term of agent demographics, the considered MABMs do not incorporate an evolving age structure of individuals and keep the number of agents of different types constant over time. Firm entry occurs in most models only after a firm has exited the market and thereby is essentially a replacement of the exiting firm (with different firm characteristics and equity). Only the AGH model captures, in a rather sophisticated way, the market entry of firms and the associated decisions of potential entrants as well as financial transactions. A simple endogenous firm entry process, in which the (stochastic) number of entrants in a sector depends positively on the number of incumbents in the industry as well as on the sectoral ratio between firms' liquid assets and debt, has also been incorporated in a recent version of the KS model (see Dosi et al. (2017b)). Although the possibility to consider spatial structures and (emerging) heterogeneities of distributions of agents characteristics across regions arguably is a merit of the agent-based modeling approach most of the models so far have not considered multi-regional settings. Exceptions in this respect are EUBI and LAGOM, which have been used for analyses fo economies with multiple regions or countries. Recently, also multi-country versions of the KS (Dosi et al. 2017c) and the EUGE model (Petrovic et al. 2017) have been put forward.

Considering the consumption goods market, the focus of the different models, in particular whether they aim to explain endogenously economic growth and technological change, shows clearly in the way the production process is covered. Whereas, KS and EUBI incorporate vintage structured capital goods and endogenous vintage choices of firms, the other MABMs consider capital with homogeneous productivity ${ }^{78}$, which is typically combined in fixed proportion with labor to produce the consumption good. As discussed in the previous section, assuming mark-up pricing is most common, where the mark-up is typically adjusted over time based on the firms' market share or its inventory level (relative to expected de-

\footnotetext{
${ }^{78}$ In the version of EUGE in Raberto et al. (2018) capital is differentiated with respect to its energy efficiency.
} 
mand). Only EUBI features a different approach in which firms explicitly estimate the form of the demand function they are facing. Quantity choices in most models are determined by the expected demand (typically adjusted by a security buffer and the current stock of inventory), in JAMEL and LEN the output quantity is determined by the size of the labor force, which however is adjusted over time based on the relationship between expected demand and inventory.

The size of the physical investment of consumption good firms is in most models simply derived from the potential need of expansion due to the current production plan. In these investment rules long run planning has no effect on the investment patterns, which is arguable rather myopic, since the acquired physical capital will be available for the firm for an extended period of time. An exception in this respect is CATS, where investment is based on a target level of capital which is calculated from past capital requirement over an extended time interval. Whereas this rule seems less myopic it still does not involve any explicitly forward looking planning of the firm. Harting (2015) has developed an extension of the EUBI model, in which investment is determined in a forward looking way using a real-option approach. Also, in most MABMs the firm's demand for inputs (capital and labor) are dictated by the decision on the scale of activity, which is typically driven by observed past demand for the goods produced by the firm. Potential changes in the price of the different inputs do not play any allocative role. For instance, the demand for capital (labor) by a C-firm is determined by the expected sales of the firm, which in turn is determined by the (past) consumer's demand. Changes in the price of capital (labor) hence do not enter the considerations determining the current demand for these inputs. Exceptions in this respect are EUBI, where the estimated unit costs of production, which are determined by input prices, influence price and quantity decisions, and EUGE, where, although input prices do not influence the planned production level, they determine the desired capital-labor ratio.

With respect to the determination of the households' consumption budget, (piecewise) linear rules based on the wealth and income have emerged as the dominant choice in the design of MABMs. In particular, a large fraction of model relies on buffer stock rules put forward in Carroll and Summers (1991), which also have strong empirical and theoretical foundations. Also the matching between households and consumption good producers is modeled in similar ways across the considered models, in which explicit protocols for this matching procedure are given. Consumers observe the prices of a randomly selected subset of consumption good producers and then select (with high probability) the cheapest ones. If they cannot purchase the full amount they demand, households move to the next best option and so on. A different approach in this respect is chosen in AGH, where customers have evolving networks of stores to which they have relations and then buy from these firms in a way to maximize their utility.

The capital good markets in the considered MABMs typically have a much simpler structure than the consumption goods markets, since it is usually assumed that there is no explicit quantity choice of producers who simply produce based on orders they have received. Also, apart from the KS and the CATS model, no competition between capital good producers is considered. With respect to pricing there is a large heterogeneity of the considered pricing rules including fixed mark-up rules, prices based on anticipated value of the capital good for consumption good producers and pricing rules based on expected demand and inventory similar to approaches used for the consumption good market. In the EUBI and the KS model endogenously evolving sets of multiple vintages of capital goods are considered. Whereas in the EUBI model new vintages emerge randomly, the KS model incorporates an 
explicit representation of the innovation activities in the capital goods sector such that the probability of a firm to innovate depends on its R\&D investments.

Also with respect to the interactions on the labor market there is considerable uniformity across the MABMs. Labor demand is derived from the planned output and with the exception of Dosi et al. $(2017 d, b)$ none of the considered models explicitly captures inertia in the adjustment of the stock of workers that might for example be driven by labor market regulations or labor contract durations. ${ }^{79}$ The majority of MABMs relies on random search of job-seekers in combination with posted wages of firms having vacancies, where posted wages are adjusted if the searching firms are unable to fill their vacancies. In several MABMs (EUBI, EUGE, KS, LAGOM) wages are also adjusted according to the evolution of labor productivity. In EUBI and EUGE the productivity of a worker is strongly influenced by her skills, which evolve endogenously over time. No such skill differentiation is present in the other (standard) models although a recent version of the KS model also exhibits heterogeneous and endogenously evolving skills of workers (Dosi et al. (2017b)). Furthermore, dynamically adjusting reservation wages, which rely mainly on the worker's current (wage or unemployment) income, determine in several of the models, whether workers accept the jobs with the posted wages.

External financing of firms is in all considered models, with the exception of EUGE, restricted to bank loans. With respect to the interest rate the banks charge to firms, the majority of MABMs follows an approach where a mark-up, whose size depends on the borrowers leverage respectively riskiness, is applied to the Central Bank interest rate. On the other hand, there is substantial heterogeneity across the models with respect to possible rationing of firms on the credit market. Whereas in some MABMs all credit demands are satisfied (JAMEL, LAGOM), others introduce probabilities of loan approvals (AGH, CATS) or assume (regulatory) upper bounds on the volume of credit a bank can grant (EUBI, EUGE, $\mathrm{KS}$ ). A common feature of the credit market in combination with the investment rules in the considered models is that firms do not take into account the induced interest payments when deciding on their output and investment targets, although the amount of credit they need influences their financial standing and thereby their interest rate and size of interest payments. Hence, the impact of the central bank base rate and of the shape of the interest rule the banks use typically is weak in these models. With exception of the Eurace models none of the MABMs includes a stock market. Also in EUBI only a minimalistic stock market is considered in which an index share, containing all firms in the economy, is traded. EUGE incorporates a more complex stock market in which different shares are traded.

Only a subset of the considered models assigns an active role to the government, and also in these models government activity essentially reduces to collecting income and profit taxes in order to be able to pay out unemployment benefits and potentially implement additional fiscal policy measures, like paying out firm subsidies. In all these models tax rates are dynamically adjusted over time to fulfill a balanced budget or a target debt to GDP ratio. Different variations of the Taylor rule are the dominant choice to determine the central bank interest rate, however in several of the MABMs the interest rule is also treated as fixed model parameter.

This brief discussion highlights that in spite of the heterogeneity with respect to the agenda and objective of the different MABMs a consensus has emerged in the MABM com-

\footnotetext{
${ }^{79}$ Frictions of such type have been considered however in Goudet et al. (2015) in the framework of an agent-based labor market model.
} 
munity about a number of key features of behavioral rules and interaction protocols. A similar statement can certainly be made about empirical validation of the models. The reproduction of stylized facts has become a standard way to show that the developed MABMs are able to capture important economic mechanisms that are responsible for business cycle properties and distributional characteristics in real economies. As Tables A1 and A2 clearly show, many of the MABMs have been very successful in qualitatively matching stylized facts on different levels of aggregation, combining the reproduction of average levels of key variables with time series properties and distributional patterns. Clearly this point is one of the perceived main strengths of the agent-based approach in (macro-)economics, although it has to be observed that clear quantifiable standards for what is exactly meant by the 'reproduction' of a stylized fact is so far missing. The last few years have seen a significant increase in the work trying to quantify the quality of the match between simulation output generated by agent-based models and empirical data (e.g. Guerini and Moneta (2017), Barde (2017)) as well as approaches for systematic estimation of agent-based models in Economics (e.g. Grazzini et al. (2017)). This work is discussed in more detail in the Chapter [Lux/Swinkels] of this Handbook and we refrain from a detailed treatment here.

\section{Policy Analysis}

Early work in agent-based macroeconomics, as well as in agent-based studies of individual markets, was mainly concerned with showing that agent-based models are able to capture important empirical features of real world economic dynamics and with exploring implications of different assumptions about individual behavior and interaction schemes. The last ten years, however, have seen a considerable increase in agent-based work with a policy focus. Several reasons might have contributed to this development. First, the financial and economic crisis unfolding after 2008 highlighted the need of building policy analyses on models which are able of capturing mechanisms which are potentially responsible for the outbreak of crises, as well as describing economic dynamics far off an equilibrium state. In particular, the interplay between real and financial markets, contagion effects, dynamic expectation formation and similar issues came to the forefront of attention for policy makers, and clearly agent-based models are well suited to deal with these issues. Second, the work in agent-based macroeconomics has seen some consolidation in the sense that a number of models (many of them have been discussed in the previous sections) have been systematically developed and improved over a considerable time span. Improved confidence in the ability of these models to capture key economic mechanisms, e.g. by building on their ability to reproduce a wide range of stylized facts, has also made them a more reliable basis for policy analysis. Some of these models have been developed into something like 'workhorse' models for policy analysis and have been used for the exploration of the effects of policies in a diverse range of domains. Third, the accumulating evidence in Experimental and Behavioral Economics that individual behavior is often not determined by (intertemporal) optimization and rational expectations has generated increasing attention in macroeconomics for studying learning and adaptive expectation formation and to investigate policy effects in models capturing these phenomena. Several of the agent-based contribution to the policy literature are addressing this point.

Apart from these developments, several other well-known properties of the agent-based approach models seem particularly useful in the context of policy analyses. This includes 
the ability to distinguish explicitly between short-, medium- and long-run effects of policies as well as to capture potential path dependencies and hysteresis effects triggered by policies. Furthermore, the fact that agent-based models are designed to represent the evolution and emergence of heterogeneities of different kinds makes them well suited to generate insights about the implications of different policies for the distribution of key economic indicators and therefore also for studying inequality in the economy.

In the following subsections we briefly review agent-based policy analysis in different key policy domains. We have decided to organize the discussion of existing policy studies in this way although several of the covered papers combine the analysis of different types of policy measures, as well as their interaction, in a unified framework. Hence, several papers will appear in more than one of the following subsections. Having discussed several contributions in different policy areas we will conclude this section with some general comments about how agent-based policy analyses have developed over the last decade.

It should be stressed that we restrict our coverage to contributions which analyze policy effects in the framework of macroeconomic models. For several of the policy domains covered there is also a very active agent-based literature relying on partial models, e.g. addressing issues of systemic risk. Furthermore, also in some areas outside the domains, which are covered in the following sections, policy related work using an agent-based approach has been done. This includes in particular the area of environmental and climate policy, where some recent contributions develop integrated assessment models linking the approaches developed in agent-based macroeconomics with some climate module capturing dynamics and effects of climate change. These models seem to have high potential for the analysis of different types of climate-relevant policies. A survey over this emerging literature can be found in Balint et al. (2017).

\subsection{Fiscal Policy}

An early analysis of fiscal policy effects in the framework of an agent-based macro-model is carried out in Russo et al. (2007). The main agenda of this paper is to compare the effect of demand oriented versus technology oriented fiscal measures in the presence of endogenous technological change. A simple macro-economic model containing only goods and labor markets is considered with random matching protocols on both markets (incorporating loyalty effects on the labor market) and simple adaptive behavioral rules governing price and wage adjustment. Production technology is linear with labor as the single input and the firm specific labor productivity stochastically evolves over time where the expected increase depends on the firm's R\&D investment. Since it is assumed that firms invest a fixed fraction of their profits in $R \& D$ the model exhibits a positive relationship between firm sales and the speed of technological change.

After the discussion of emergent properties of the model and of several empirical stylized facts it is able to reproduce, Russo et al. (2007) focus on a computational experiment aimed at exploring the qualitative consequences of alternative fiscal policies. In particular, they consider two scenarios in which a public agency introduces a (flat) tax rate on firms' profits. In the first scenario these tax revenues are paid out as unemployment benefits, whereas in the second scenario they are redistributed to firms on a per-capita basis increasing their R\&D expenditures. The simulations show a strongly diverging effect of the tax on the average growth rate in these two scenarios. In the first scenario, in which the tax is used to boost demand, the effect on growth is clearly negative and becoming stronger the higher 
the tax rate is. In the technology oriented scenario a positive effect on growth is detected, which grows with the tax rate as long as the rate stays below an upper threshold. These results show that in the considered setting the multiplier generated by higher demand is not sufficiently large to outweigh the direct negative effects of the tax on R\&D spending, which hinges on after-tax profits. The main driver of the positive effect of a technology oriented redistributive tax policy seems to be the intensification of $R \& D$ activities per se rather than the redistribution between firms. This is demonstrated by comparing the effect of an increase in tax rate with a scenario without the tax in which firms increase their R\&D rates by the same amount. It is shown that the growth rate in the second scenario, referred to as private R\&D scenario, is always above that in a corresponding public $R \& D$ scenario, in which $R \& D$ is entirely financed through the tax. This suggests that the tax per se has a negative effect on economic performance. The question whether it is needed to induce the desirable level of R\&D expenditures cannot be addressed in the considered setting since the R\&D rate is a given exogenous parameter rather than an endogenous results of firms' decision making.

Whereas in Russo et al. (2007) the positive effects of fiscal policy measures are rather limited, most of the following agent-based fiscal policy analyses come to much more positive conclusions about the effectiveness of fiscal measures, both with respect to fostering growth and to reducing business cylce fluctuations. Haber (2008) uses the agent-based macroeconomic model 'AS1', incorporating geographical structure, evolving product ranges, a rather sophisticated banking sector and explicit expectation formation processes, to analyze the effect of an expansionary fiscal policy on key economic indicators. In particular, the implications of a reduction of the tax rate on income and firm profits is studied. It is shown that such a reduction leads to an increase in economic growth as well as a reduction in unemployment and increase in inflation. Using the fact that the model can capture a variety of different expectation formation rules, Haber (2008) also demonstrates that the positive effect of the fiscal expansion is reduced if the agents employ more sophisticated foresight mechanisms. In any case, the reduction of the tax rate induces a higher level of public debt, which in the framework of the model is covered through bank credit.

The potential growth enhancing effect of an expansionary fiscal policy has also been an important theme in the analysis of fiscal measures in the framework of the 'Keynes meeting Schumpeter' (KS) model. In Dosi et al. (2010) the effects of a rise in the level of unemployment benefits financed by an increase of the tax rate on firm profits is studied. It is shown that increasing the rate of unemployment subsidies relative to wages (and therefore also the tax rate) from zero to a positive level has a significant positive effect on the growth rate of productivity and output, where the actual size of the positive rate has only little influence. The authors explain this observation in a way that in the absence of unemployment subsidies the low demand negatively affects firms' R\&D investment and thereby also negatively affects productivity growth leading to a vicious cycle associated with low growth and strong fluctuations. A minimal level of fiscal stimulation is needed to avoid such a low growth scenario and to allow the economy to fully exploit the technological opportunities for productivity growth arising in the considered framework. Whereas the level of a positive unemployment and tax rate hardly affects the speed of growth, increasing these rates leads to reduced output volatility and reduced unemployment. This analysis is extended in Dosi et al. (2013), where it is shown that the positive effects of such a fiscal policy, which redistributes firm profits to households, is larger the more market power the firms have. More precisely, the authors establish that the reduction in volatility and unemployment induced by higher unemployment subsidies respectively tax rates is more pronounced if firms charge a high price mark-up on 
consumption goods. If mark-ups are so low that the economy is persistently in (almost) full employment an increase in the unemployment subsidy/tax rate yields an increase of growth rate volatility. These observations reinforce that in the framework of this model the main role of the considered fiscal measures is to channel funds from firm savings to households, where it stimulates demand. The authors stress that their results highlight the importance of the interactions between income distribution regimes and redistributive fiscal policies.

The observation in Dosi et al. $(2010,2013)$ that fiscal measures can reduce economic fluctuations illustrates a theme that has been an important motivation of numerous agentbased studies of fiscal policy, namely that this type of policy models are well suited to examine the mechanisms by which fiscal policies might trigger or avoid crises and to explore potential fiscal policy responses to crises once they have emerged. In Dosi et al. (2015) the dynamics of the KS model in the benchmark case with constant unemployments subsidy/tax rate is compared to scenarios in which these rates are adjusted according to rules resembling the European Stability and Growth Pact (SGP) and the European Fiscal Compact (FC). In particular, under these rules the level of unemployment subsidies is reduced if the deficit is above $3 \%$ of GDP, in case of SGP, or, in case of FC, public expenditures have to be cut also if public debt exceeds the target of $60 \%$ of GDP. For both types of rules also an adjusted version with escape clause is considered, in which these constraints do not apply in periods in which the growth rate is negative. The simulation results clearly indicate that compared to the benchmark both rules without escape clauses have significant and large negative effects on growth and employment. Furthermore, they strongly increase output volatility and the likelihood of economic crises. Due to these negative implications for the level of economic activity in the framework of this model the considered rules even induce exploding dynamics of public debt to GDP ratios. In the presence of escape clauses the negative implications of these fiscal rules are much weaker, however, with the exception of the effects on the growth rate, still significant. The authors conclude from these observations that an unconstrained fiscal policy allowing automatic stabilizers is needed to dampen economic fluctuations and to reduce the likelihood of crises. They also discuss the interplay of such a policy with different variants of monetary policies (see Section 4.2).

The results of Dosi et al. (2015) that fiscal contraction in bad times yields negative implications for recovery and growth is consistent with evidence presented in the framework of other agent-based macroeconomic models. A study closely related to that of Dosi et al. (2015) is carried out by Teglio et al. (2018) in the framework of the Eurace simulator (EUGE). They also consider the effects of fiscal rules, corresponding in a stylized way to the European Stability and Growth Pact (SGP) and the European Fiscal Compact (FC), and combine them with an escape clause and a 'fiscal accommodation' (reduction of tax rates during crises) as well as with quantitative easing by the central bank. Similarly to Dosi et al. (2015) they find that applying escape clauses and fiscal accommodation both to the SGP and the FC reduces the probability of depressions and increases average growth rates. Napoletano et al. (2015) study in the framework of a simple endowment economy with a credit market the effectiveness of different fiscal policies in the aftermath of a (household) bankruptcy shock. They show that a deficit-spending rule is able to reduce the negative impact of the bankruptcy shock on the economy and to favor a fast recovery from the recession more effectively than balanced-budget policies. Furthermore, the size of the fiscal multiplier is time-varying and is largest in the downturn several periods after the shock. Neveu (2013) uses a model, which in structure is similar to that of Russo et al. (2007), however with a slightly different fiscal setup. Fiscal revenues are generated by a combination of a corporate 
profit tax and an progressive income tax and are spent partly on unemployment benefits and partly on R\&D subsidies for firms, which are distributed across firms proportionally to last period profits. Three fiscal rules are considered, in which special measures are activated once the economy contracts for four successive periods: i) fiscal spending is increased while the tax rate is kept constant; ii) spending and taxes are cut such that a balanced budget is maintained; iii) the tax rate is reduced while spending is kept constant. Under the first and the third rule public debt is accumulated during downturns, and during growth periods taxes are adjusted to repay this debt. It turns out that all three rules have no significant effect on average growth or unemployment, however, differently from rules i) and ii) the third rule reduces the number of long downturns significantly and substantially. Hence, a debt financed stimulation of demand and R\&D during bad times helps to avoid long and deep recessions without negatively affecting growth perspectives even if the debt accumulated during downturns is repaid during growth periods.

Similarly to Neveu (2013), also Harting (2015) studies the implications of different fiscal responses to downturns on the growth rate and volatility of the economy. In the framework of an extended version of the Eurace@Unibi model (EUBI) the following three scenarios are compared, where in each a special fiscal policy is activated once the growth rate of output drops below a given threshold. In the first scenario households receive a direct fiscal transfer thereby increasing their consumption budget, in the second scenario all firms receive subsidies on any investment they make, whereas in the third scenario subsidies are paid out only to firms investing in the best available vintage of the capital good. Since in the Eurace@Unibi model the vintage choice of firms is determined by the comparison of the ratio of expected future return to price of each vintage, the third rule influences the technology choice of firms and fosters the acquisition of frontier vintages. Main insights from the analysis in Harting (2015) are that all three policies are able to reduce the volatility in the economic dynamics. However in order to achieve the same reduction of volatility the demand oriented first measure requires substantially larger fiscal expenditures during the downturns than the other two technology oriented rules. Furthermore, the three rules differ substantially with respect to their growth effects. No such effects are induced by the second measure, i.e. the payout of subsidies to all investing firms. The other two rules have positive growth effects and these effects are much stronger if the third rule is used, in which only firm investment in frontier technology is subsidized. These results imply that the facilitation of diffusion of new technologies in the economy is the main driver of the positive effects of these policies.

While the papers reviewed in the previous paragraphs have focused on the role of fiscal policy measure as automatic stabilizers and as instruments to prevent crises and economic fluctuations, Dawid et al. (2018b) examine which types of fiscal measures are most effective in fostering recovery and growth in a region which is in an economic crisis. The agenda of the paper is motivated by the developments associated with the public debt crises in southern European countries unfolding after 2010. This paper uses a two-region version of the Eurace@Unibi (EUBI) model and considers a scenario where the two regions are in an economic union with integrated consumption and capital goods markets but differ substantially with respect to size as well as the technological level of firms and the (specific) skill endowment of workers. In particular, a scenario is considered in which the smaller and technologically lagging region has accumulated so much public debt that it no longer has access to the credit market. Without any fiscal transfers from the larger and faster growing region this situation would lead to a rigorous decline in output and consumption in the small region. The paper compares the effects of different union-wide financed fiscal 
transfer schemes to the crisis region and shows that technology oriented subsidy policies, which are able to positively influence the technology choice of firms in the lagging region is the only option to foster long-run catch-up of the that region. The effect of this policy becomes significant only after a considerable delay, whereas measures strengthening demand in the lagging region, have a positive short run effect on consumption in that region, however with little positive impact on production and productivity. A policy in which only the debt burden of the lagging region is taken over by the union has no significant positive effect on the economic dynamics in that region. The paper shows that under none of the considered policy measures the larger and stronger region in the union, which covers the majority of the fiscal expenditures, experiences significant negative effects on consumption or production.

\subsection{Monetary Policy}

A strong focus of agent-based work with a policy orientation has also been on monetary policy issues and the optimal design of regulatory schemes for banks and the financial market. We will survey contributions concentrating on regulatory issues in the next subsection and concentrate here on studies of classical monetary policy instruments, in particular interest rate rules.

Dosi et al. (2013) study the implications of a variation of the (constant) central bank interest rate in the framework of the KS model. In their setting a variation of the interest rate in the range between $0 \%$ and $10 \%$ essentially has no impact on the level or on the fluctuations of growth rates. Only as the rate is increased beyond this level at some value, which depends on the firms' market power, a strong negative impact on the growth rate associated with increasing fluctuations emerges. For sufficiently high levels of firm mark-up such negative impact of interest rate increases is however avoided. The intuition underlying these results is that the interest rate negatively affects growth performance mainly by increasing the danger of firm bankruptcies, which destabilize the economy, and by reducing the frequency of full employment states. Under sufficiently high mark-ups the risk of bankruptcies is negligible and hardly affected by the interest rate. Hence the effect of interest rate hikes are very minor.

While Dosi et al. (2013) assume a fixed central bank interest rate, the majority of contributions rely on variations of the Taylor rule to model the central bank's interest rate policy. Raberto et al. (2008) demonstrate that a simple output gap oriented interest rule, at least if it is sufficiently strong, leads to better economic performance in terms of output and worker utility compared to a random interest rule. Relatedly, Delli Gatti and Desiderio (2015) compare the performance of an economy under a fixed central bank rate and an output and inflation oriented Taylor rule in the aftermath of a negative productivity shock. They show that with a Taylor rule the decrease in output is much weaker and the price level stays lower compared to the fixed interest scenario. The main positive effect of the Taylor rule in this setting is that it helps to keep the firms' credit demand and liquidity higher than under a fixed central bank rate. Ashraf et al. (2016) consider in the framework of the AGH model a Taylor rule based on inflation and output gap and study the implications of a variation of the central bank's (fixed) inflation target on output, inflation and volatility. Quite in accordance with the insights in Dosi et al. (2013) they find that increasing the inflation target above a certain threshold leads to a deterioration of output and an increase in volatility. The interpretation of Ashraf et al. (2016) for these results is that a large inflation target induces a large dispersion of relative prices increasing the frictions on the goods market and thereby 
the bankruptcy risk of sellers.

Several agent-based studies have explored how changes in the set of target variables as well as in the values of the reaction coefficients in the Taylor rule affect the emerging economic dynamics and also how these effects depend on various properties of the considered economy. Dosi et al. (2015) compare the economic dynamics emerging in the KS model if the central bank targets only inflation with that under a Taylor rule with dual mandate reacting to inflation and unemployment. They find that the dual mandate rule significantly increases the growth rate in the economy and that the positive growth effect is particularly strong if a restrictive fiscal policy is in place. This growth effect also is associated with a reduction in unemployment. Furthermore, the dual mandate leads to less GDP volatility compared to a purely inflation oriented monetary policy. The authors explain the positive effect of the dual mandate rule by a better performance of the banking sector, reducing the rate of bank failures and increases the number of investment projects that are financed and implemented.

Contributions by Krug (2015) and Chiarella and Di Guilmi (2017) use different agentbased macroeconomic models to examine in how far a Taylor rule, which takes into account some indicator of economic instability, can improve economic performance and stability. Krug (2015) develops an agent-based macromodel, which combines a simple real sector with exogenous growth with a quite detailed representation of the money market and the financial sector. He examines the implications of an extension of a dual mandate Taylor rule (inflation and output gap), which either reacts also to the deviation of a financial stability indicator from its target value or reacts also to the deviation of the credit to GDP ratio from its long term trend. A main finding of the paper is that also with such an extension of the policy rule, monetary policy alone cannot achieve significant improvements with respect to financial stability if the goal of macroeconomic stability remains the main focus. Combining a dual mandate Taylor rule with macro-prudential policy in form of a more rigid regulation of the financial sector seems much more efficient in this respect. The paper highlights also that implementing a "leaning against the wind" monetary policy (i.e. a Taylor rule reacting with a central bank rate increase to an increase of the financial instability indicator) in a restrictive regulatory environment is counterproductive and actually leads to higher financial fragility. Chiarella and Di Guilmi (2017) also study the effects of a policy oriented towards financial stability by considering a Taylor rule which includes the deviation of the current fraction of Ponzi firms from its past moving average as a financial instability indicator. Motivated by the seminal work of Minsky (1963) firms are classified as Ponzi if they have positive debt and negative profit. Quite in accordance to the main insights from Krug (2015) they also find that increasing the (positive) reaction coefficient of the central bank rate to the fraction of Ponzi firms leads to a larger rather than a smaller fraction of Ponzi firms in the economy and does not reduce the bankruptcy rate. Furthermore, such a change in the monetary policy has a negative impact on aggregate output.

While the work discussed in the previous paragraph has highlighted the suitability of an agent-based approach to study the interplay of monetary policy and regulation, work by Salle et al. (2013) and Salle (2015) exploit the ability of this approach to capture heterogeneous expectation formation processes and learning in a macroeconomic framework. They consider a macroeconomic model with social learning among households and individual learning of firms in which the central bank uses a non-linear Taylor rule responding to inflation and unemployment. Salle et al. (2013) examine the impact of an increase of the reaction coefficients of inflation and unemployment under different credibility scenarios for the central bank. They find that monetary policy is much more effective if the central bank's target 
rate can be communicated with full credibility and is perceived without noise. In Salle et al. (2013) the inflation expectations of households are formed in a very simple way as a weighted average between the perceived inflation target and the past inflation. In Salle (2015) a much more sophisticated and flexible expectation formation process is inserted into this macroeconomic model. This process is modeled using artificial neural network with different sets of input variables which vary according to the considered transparency scenario. These input variables might also include the central banks internal inflation forecasts, based on VAR models, or the trend of these forecasts. The analysis of extensive simulation exercises under the different scenarios shows that transparency of the central bank reduces the trade-off between the goals of inflation targeting and minimization of the output gap. In particular, it turns out that a central bank which communicates not only its objectives but also its forecasts reduces the inflation-output gap trade-off and should use smaller reaction coefficients to these two objectives than a central bank restricting communication to its objectives.

In addition to the extensive work studying the impact of different interest rate rules of the central bank on economic performance, agent-based models have also been used to explore the macroeconomic effect of quantitative easing. Cincotti et al. (2010) use the Eurace simulator (EUGE) to compare quantitative easing scenarios, in which the central bank purchases government bonds, thereby financing public deficits, with fiscal tightening. Under fiscal tightening the deficit of the government is funded by issuing new bonds in the market and by an increase of tax rates. The effects of the two policies are compared under different assumptions about the dividend payout ratio of firms, where higher dividend ratios imply a larger demand of firms for external credit and therefore a higher amount of credit in the economy. The authors show that quantitative easing leads to higher growth rates in the economy as well as to higher inflation compared to the fiscal tightening scenario. The effect is much stronger in scenarios with a relatively small dividend payout rate. Also Chiarella and Di Guilmi (2017) combine their analysis of variations of the Taylor rule, which was discussed above, with policy experiments based on a central bank rule determining the supply of money. In particular, the supply of central bank money is assumed to depend negatively on inflation and on the number of Ponzi firms in the economy. In the exogenous money scenario, in which only the central bank, but not the financial sector can generate money, an increase of each of the parameters capturing the effect of inflation respectively Ponzi firms on money supply reduces the number of Ponzi firms. In the endogenous money scenario, in which the financial sector endogenously generates all credit demanded by firms and liquidity demanded by investors, increasing the sensitivity parameter with respect to inflation also reduces the variance of output fluctuations.

\subsection{Financial Regulation and Crisis Resolution Mechanisms}

On of the main policy lessons learned from the financial and economic crisis of 2007 is that systemic risk matters and should be taken into account when designing regulatory policies. This has not only lead to the development of the Basel III framework for the regulation of the financial sector, but also to an explosion of academic work studying the implications of different regulatory schemes from a systemic perspective, which acknowledges the interconnectedness of the players in the financial sector as well as the feedbacks between the real and the financial sector. An agent-based approach seems a natural choice to transform such a perspective into concrete models and indeed a number of contributions based on such an approach have improved our understanding of the macroeconomic effects of different regulatory 
instruments discussed in the aftermath of the crisis.

Teglio et al. (2012) use the Eurace simulator (EUGE) to analyze the effect of making the regulatory capital requirement, defined in terms of the ratio of equity to risk-weighted assets, more restrictive. They find that a very strong requirement, which implies some credit rationing of firms from the start of the simulation, has negative implications for short term GDP in the economy, however induces higher output und lower unemployment in the long-run compared to a looser regulatory scheme. They argue that under a more restrictive scheme the buildup of financial fragility of firms is avoided, which leads to more significant credit rationing of firms in the long run under loose regulatory schemes. In Cincotti et al. $(2012 b)$ this analysis is extended by considering capital requirements which are adapted over time in a countercyclical way, i.e. they become more tight in good times compared to bad times. Two versions of such rules are considered, where the level of unemployment respectively the amount of total credit are used as indicators of the state of the economy. Simulations indicate that in the long run output is higher and unemployment is lower under such adaptive policies compared to fixed capital requirements. This effect is particularly strong if the countercyclical policy is based on total credit in the economy. Raberto et al. (2018) explore in the framework of the Eurace simulator (EUGE) how a capital adequacy requirement for banks, which requires lower reserves for firm loans compared to household mortgages, might foster firms investment in new technologies and the transition to green energy production. They find that such regulatory schemes in the short run indeed speed up firm investment in new technologies, but at the same time reduce overall growth due to stronger constraints on household expenditures. In the long run this negative effect outweighs the positive one even with respect to firm investment.

van der Hoog and Dawid (2017) pose the question which type of banking regulation is best suited to avoid large downturns in an economy. Within the framework of the Eurace@Unibi model (EUBI) they use indicators developed in the empirical literature to identify amplitude and duration of recessions and expansions in the simulation data and analyze how the distribution of these indicators depends on the regulatory environment. In particular, they compare the effects of strengthening the capital requirement with those of making the reserve requirement, which focuses on a bank's liquidity, more stringent. They find that if the capital requirement becomes more restrictive the distribution of recession amplitudes shifts in a way that the amplitudes of the most severe recession become larger and overall strong recessions become more likely. If the reserve requirement becomes more restrictive an opposite effect is observed and the distribution of amplitudes becomes more concentrated around smaller values. Based on their examination of the mechanisms underlying these observations, which involves the categorization of firms according to their financial status, the authors argue that a liquidity constraint is able to prevent banks from fueling a debt bubble, that is mainly caused by the financially unsound firms requiring new debt to roll-over old debt. Hence, strict liquidity requirements reduce the risk of large downturns. Relatedly van der Hoog (2018) shows, again in the framework of the Eurace@Unibi (EUBI) model, that a stringent reserve requirement reduces the output fluctuations without negatively affecting the (average) dynamics of total output.

An extensive agent-based analysis of the effect of different types of regulatory schemes for banks is also carried out in Popoyan et al. (2017). They build their analysis on an extension of the AGH model, in which the banks' rule for granting loans to shops is based on a frequently used approach for determining creditworthiness, the ' $6 \mathrm{C}$ ' approach. The main objectives of the study are to compare the economic dynamics under a Basel II type 
regulation of banks with that under a Basel III approach, and to analyze which aspects of the Basel III regulation are most crucial for the effectiveness of that scheme. In particular, the paper distinguishes between the following four regulatory instruments, which are all part of the Basel III scheme:(i) a static capital requirement; (ii) a countercyclical buffer, as an add-on to the capital requirement, which is determined based on the aggregate private sector credit-to-GDP ratio; (iii) a leverage requirement; (iv) a liquidity requirement. Considering the level and the volatility of output gap and unemployment as well as the likelihood of crises the authors find that the Basel III scheme performs significantly better than Basel II. More specifically, the combination of the static capital requirement with the countercyclical buffer seems to be the crucial aspect of the Basel III approach. Implementing just these two instruments already yields results that are only slightly worse than those under the full Basel III package. However, applying only the leverage requirement or only the liquidity requirement produces significantly larger output gaps and unemployment values as well as more volatility. The authors show that in their setting these instruments alone perform worse than the Basel II rule. These claims are verified across different Taylor rules that are inserted into the model. Comparing these findings with those of van der Hoog and Dawid (2017) and van der Hoog (2018), discussed above, one has to acknowledge that, whereas the importance of the countercyclical buffer seems consistent with the intuition of the importance of bubble prevention in these papers, the negative role of the liquidity constraint is at odds with the main findings in van der Hoog and Dawid (2017) and van der Hoog (2018). This observation highlights the importance of taking into account the details of the underlying macroeconomic setup when interpreting the qualitative insights from policy analyses. Riccetti et al. (2017) employ still another agent-based macroeconomic framework and show that, similarly to the finding reported above, also in their setting a too loose capitalbased banking regulation increases financial fragility with potential negative implications for the real economy. However, they also point out that an overly tight regulation, which restricts the availability of credit too strongly, is detrimental for the performance of the economy.

The papers we have discussed in this Section so far have focused on the regulation of regular commercial banks. Krug and Wohltmann (2016) point out that in recent years actors other than commercial banks, which are not subject to the corresponding regulation, have taken over a more important role of financial intermediaries in the economy. They analyze the impact of such shadow banks on the dynamics and stability of the economy in a stockflow-consistent agent-based macroeconomic model, in which banks are subject to Basel III type regulation. Inserting unregulated shadow banks into a benchmark scenario, in which only commercial banks offer financial services, the authors find a destabilizing effect of the informal financial sector on the economy. At the same time they find a small positive impact of the informal financial sector on the growth rate. If the shadow banks are also subject to regulation and share the traditional banks' access to central bank liquidity, than the variance of output as well as that of inflation, is however substantially lower than in the benchmark. Based on this, the authors argue that including the shadow banks in the regulation and giving them access to the central bank as lender of last resort is helpful for reaching the central bank's dual mandate of price and output stabilization. At the same time the average growth rate of output is substantially smaller in such a scenario with regulated shadow banks compared to the benchmark.

The regulatory environment of the financial sector is not only determined by the constraints banks face while solvent, but also by the mechanisms that apply in case a bank is defaulting. Klimek et al. (2015) compare the macroeconomic effect of three different types of 
such crisis resolution mechanisms in the framework of a simple agent-based macroeconomic model. These mechanisms are: (i) a purchase and assumption (P\&A) operation under which assets and liabilities of the defaulting bank are taken by the other banks; (ii) a bail-out under which households and firms provide equity for the bank; (iii) a bail-in under which unsecured claims of creditors are written off or transformed to equity. In all cases the mechanism is activated if the equity of a bank falls below a certain (negative) threshold. The effect of these different mechanisms on output, unemployment and credit volume is compared for different levels of the banks refinancing interest rate (which drives the size of the interest rate the bank is charging the firms). For low interest rates, the choice of the crisis resolution mechanism has little effect, but for higher interest rates bail-in and bail-out lead to better results than $\mathrm{P} \& \mathrm{~A}$. In particular, in the highest interest rate regime, which is characterized by high unemployment and low household savings the bail-in mechanism leads to highest output and lowest unemployment because in such a setting the financial burden for firms and households, implied by the bail-out of banks, significantly affects their level of economic activity reducing the overall output in the economy.

\subsection{Labor Market Policy}

The discussion in the previous subsection highlights that, in particular after the crisis of 2008, the focus of the (agent-based) literature dealing with market regulation has been on the financial and credit market. However, in the framework of agent-based macroeconomic models also the effects of regulatory schemes on other markets, in particular the labor market has been studied. An example in this respect is Seppecher (2012), who in the framework of the JAMEL model, shows that higher flexibility of (reservation) wages decreases unemployment duration, but through the demand channel reduces consumption and real wages and increases unemployment. ${ }^{80}$ The introduction of a minimum wage in such a scenario can help to eliminate such negative effects of increased wage flexibility.

The findings of Seppecher (2012) are consistent with results obtained in the framework of other agent-based studies, like Napoletano et al. (2012) and Dosi et al. (2017d). In the later paper the authors compare the effects of different labor market regimes in the framework of a version of the KS model with a decentralized labor market. A 'Fordist' regime, in which wages are indexed to productivity, firms do not dismiss workers as long as profits are positive and workers do not search on the job, is compared to different variations of a 'Competitive' regime, in which wage changes respond to unemployment, firms immediately adjust the size of their work force to their current production plan and workers search on the job. The main insight from their analysis is that in scenarios, where growth is mainly driven by demand the Competitive regimes no full indexation of minimum wages and no unemployment benefits are associated with higher unemployment, slower growth and a higher risk of economic crises compared to the Fordist regime. The main mechanism underlying this observation is that under the Competitive regimes without wage indexation wages and thereby demand are strongly diminished during downturns, which reduces firm investment in R\&D and new vintages, which reduces the rate of productivity growth. In a similar setting and following a similar logic Dosi et al. (2018) show that the Fordist regime also induces lower

\footnotetext{
${ }^{80}$ It should be pointed out that we cover here only papers using macroeconomic agent-based models. There are also several studies of the implications of changes in the labor market design using partial agent-based model of the labor market, see e.g.Haruvy et al. (2006), Goudet et al. (2015) or the survey in Neugart and Richiardi (2018).
} 
unemployment and lower income inequality than alternative regimes under which firms have higher flexibility in dismissing workers. In Dosi et al. (2017b) the Fordist and Competitive labor market regimes are compared with respect to the question how much hysteresis an economy exhibits under these regimes. The model of Dosi et al. (2017d) is extended by incorporating endogenous skill dynamics of workers into the model and also introducing endogenous firm entry and the main focus of the analysis is on the question how strong and how long lasting the negative impact of a crisis on future growth is. Quite consistent with the results of the two papers just discussed the authors find that under a Fordist regime the negative impact of a crisis is substantially less severe and also shorter-lived than under a competitive regime.

In Dawid et al. (2013) the effect of increasing the flexibility of the labor market is studied in a two region version of the EUBI model in which the two regions differ with respect to (initial) firm productivity and worker skills (see also Section 4.5). In particular, it is studied how the growth and inequality in the weaker region is affected if the wage replacement rates in that region are decreased and the pressure to find work are increased such that the workers' reservation wages adjust downwards much faster if they are unemployed. Whereas such measures induce a decrease of the growth rate of output in that region it also reduces the income inequality in the region. Both effects arise because under a more flexible labor market technologically less advanced firms in the region have better chances to hire workers who have been earning higher wages at firms with higher productivity before. This reduces the wage dispersion in the population and at the same time increases competition for the high productivity firms reducing their investment and thereby reducing overall productivity growth.

\subsection{Regional Growth, Convergence and Cohesion Policy}

In a stream of papers different versions of the Eurace@Unibi model (EUBI) have been used to study how different types of (region-specific) policies influence the regional dynamics of growth and technological change in an economy consisting of several heterogeneous regions. In Dawid et al. (2008) a two-region version of the model is considered and it is assumed that a certain budget is available to improve the general skills, i.e. the speed by which workers can acquire specific skills that increase their productivity. The main question posed is how the effects of an equal distribution of the skill upgrading measures across the two regions compares to an approach where all effort is concentrated in one region ('light-house policy'). It is shown that the equal distribution leads to higher average productivity and output in the economy in the long run, whereas a concentration of effort induces faster growth in the short run. A crucial assumption for this finding is that labor is immobile across regions. This implies that firms in the light-house region, which due to their productivity advantages in the short run also have cost and price advantages relative to firms from the other region, face problems filling their vacancies with local workers once the demand for their products becomes too large. These rationing problems on the labor market inhibit output expansion and growth and also induce upwards pressure on wages in the light-house region. If the upgrading of skills is equally distributed across the regions such effects do not occur, which explains why such an equal distribution yields better outcomes in terms of economy wide output and productivity growth.

The importance of spatial labor market frictions for the results in Dawid et al. (2008) is highlighted in Dawid et al. (2009), where the same policy experiment is carried out under 
a completely integrated labor market of the two regions (no commuting costs for workers working in the other region from where they live) and a market with positive, but small commuting costs. Not surprisingly, in the case of a completely integrated labor market the allocation of the skill upgrading measures between regions is irrelevant, however for small positive commuting costs the opposite result to Dawid et al. (2008) is obtained. Concentrating all skill upgrading measures in one region here leads to higher economy wide output in the long run. The impact of different degrees of labor market integration on regional economic dynamics and economy wide output is studied in more detail in Dawid et al. (2012). Again, a two region version of the Eurace@Unibi (EUBI) is studied, in which the first region (the 'high tech' region) is characterized by a larger fraction of workers with high general skills and an initial advantage of the local firms with respect to the quality of their physical capital stock compared to the second region ('low tech'). Inspired by discussions about the integration of labor markets between EU member countries after the EU enlargement in 2004 the authors compare the economic dynamics emerging under four different policies: (i) full separation of labor markets; (ii) full integration (no commuting costs); (iii) integration with small commuting costs between regions; (iv) integration with small commuting costs with some delay (after the integration of the product markets). A main finding of this paper is that, whereas in terms of economy wide long run output open labor markets clearly outperform closed ones, the ranking of the different policies differs crucially depending on which region and which time horizon is considered. For the high-tech region closed labor markets are preferable in terms of the level of produced output if the planning horizon is short, while under a long run consideration open labor market with small frictions perform best. For the low tech region the opposite holds true. Closed labor markets are harmful in the short run but have positive long run implications for this region. The underlying mechanisms are again closely related to the induced labor flows across regions, the resulting pressure on wages and the associated investment incentives of firms.

A demonstration how multi-region agent-based macro-models can be used to provide insights into the effects and the effectiveness of cohesion policies aiming at the fostering of convergence between regions is given in Dawid et al. (2014, 2013). In particular, these papers incorporate policy measures resembling instruments the European Union applies as part of its cohesion policy in a two-region setting of the Eurace@Unibi (EUBI) model, in which, similar to Dawid et al. (2012), one region has an initial advantage with respect to physical and human capital endowment. In Dawid et al. (2014) the effects of two types of cohesion policies are analyzed, namely a human capital oriented policy, under which the distribution of general skills in the low tech region is improved, and a technology oriented policy, under which firms in the low tech region receive subsidies if they invest in new physical capital at the technological frontier. It is shown that in a scenario with integrated labor markets between the two regions the human capital policy fails to achieve its objective of fostering convergence between the two regions, but, quite on the contrary, affects only the output in the high-tech region positively. The output in the low tech region, which is the target of the policy, is however negatively influenced. The reason for this observation is that the human capital policy induces a flow of highly skilled workers from the low tech region to firms in the high tech region, which due to their higher productivity offer higher wages. This has second order effects on firms' investment incentives, which give rise to this result. If labor markets are separated, then the human capital policy indeed facilitates growth in the low tech region and therefore fosters convergence. Considering the effect of the technology policy it is shown that it always fosters convergence, and that the effect is stronger if labor markets 
are integrated. Dawid et al. (2013) focuses entirely on the effects of technology policies and shows that the positive convergence effect identified in Dawid et al. (2014) disappears if the policy becomes 'undirected' in a sense that investing firms in the low tech region receive subsidies even if they invest off the technological frontier. In this paper the effect of cohesion policies is not only studied with respect to the income differences between the two regions but also with respect to income inequality within each region.

\subsection{Taking stock: What is the potential of agent-based macroe- conomics for policy analysis?}

Several general observations about the use of agent-based models for policy analysis can be made based on the literature review in this section. There seems to be a clear development both with respect to content and to methodological standards. Whereas the earlier contributions reviewed above, let's say most of the papers from 2008 to 2013, have mostly focused on rather generic and traditional policy issues, like interest rate policies or demand versus supply oriented fiscal polices, many of the more recent publications explore the implications of rather detailed aspects of actual policy proposals. These contributions exploit the ability of the agent-based approach to capture institutional details of environments and policies to disentangle the implications of different aspects of programs like the Basel III regulations or the EU cohesion policies. Clearly such insights about the expected effects of details in the policy design should be highly relevant for policy makers. Similarly, the consideration of the interplay of different types of policy measures, as well as the feedback between policies, expectations and credibility of policy makers, which has been the focus of several of the reviewed contributions, seems to be an important aspect of the agent-based work in the policy domain.

From a methodological perspective, a trend towards statistical rigor in the analysis of policy effects can be observed over the last decade. The (often graphical) comparison of the means across Monte-Carlo runs of certain considered variables between different policy scenarios, which has been quite common in earlier contributions, has more or less disappeared in more recent publications. Providing meaningful statistical tests for the significance of certain policy effects, or at least confidence bands around for the considered variables under the different policy variants has become a standard in the literature. Also, more sophisticated methods have been applied, like the use penalized spline methods for the estimation (also providing confidence bands) of the dynamics of the isolated effects of certain policies in simulation models (Dawid et al. (2014, 2013, 2018b), Harting (2015)) or regression analyses of the impact of policy parameters across certain relevant parameter spaces. For the later it is essential to rely on appropriate methods for sampling the parameter space and in this respect nearly-orthogonal Latin hypercubes (Cioppa and Lucas (2007)) have been used (e.g. Salle et al. (2013)).

Important challenges certainly remain to increase the potential impact of this work for actual policy design. First, the appeal of a policy recommendation certainly increases if it is backed up not only by careful analysis within the considered model framework, but also by a clear intuition for the causal mechanisms driving the policy effects. Given the rich structure many of the agent-based models have, developing such a narrative is typically difficult, even more so if the intention is to demonstrate that the mechanisms highlighted in that narrative can actually be identified in the simulation data. Second, in many areas of application, it is common to carry out a calibration of the model based on some empirical data before 
entering the policy analysis. As discussed in the previous section, at this point the agentbased macroeconomic models employed in policy analyses are typically not calibrated in the traditional way, but are linked to empirical data through a mix of parameter estimation and matching of stylized facts. Developing more clear standards of linking these models to data could be useful to improve clarity and also credibility of the derived policy recommendations.

\section{Conclusions and Outlook}

In this chapter we have attempted to provide a systematic overview over the state of the art in agent-based macroeconomic modeling as well as over the main areas of economic policy analysis in which these models have been applied. Our survey highlights that, in spite of a substantial number of different models that have been developed in the literature, a quite significant common core has emerged with respect to the design of agents' behavioral rules and interaction protocols as well as to the approaches for testing empirical validity of the models and to carrying out rigorous and meaningful analyses of the simulation outcomes. Also, a rich stream of policy related literature relying on MABMs has evolved, addressing issues in more or less all main areas of economic policy.

Nevertheless, it is evident that agent-based macroeconomic analysis is still a very young area of economic research facing numerous challenges and also the need for consolidation. A stronger standardization towards some 'canonical' MABMs (at least for certain parts of the model) would certainly increase the comparability across obtained results and the transparency about the main mechanisms responsible for them. However, also in light of the ongoing debate in the agent-based community about the appropriate size and granularity of agent-based macroeconomic models (see e.g. Richiardi (2017)), a certain diversity of approaches is certainly desirable and should pertain. A main challenge from a methodological perspective is certainly the development of empirical calibration methods for MABMs, which are computationally feasible even for large size models. As indicated in this chapter, promising conceptual work has been done during the last few years and in light of the ever increasing computational speed it can be expected that we will see the emergence of suitable standard methods and algorithms for this purpose very soon.

From an economic perspective, several areas can be identified for which the potential payoff of using MABMs seems particularly high. As discussed above, one important area of work in this respect is the analysis of economic crises, taking into account the interplay of the real and the financial sector and the mechanisms generating systemic risk. Another topical area, for which the ability of MABMs to model the dynamic evolution of populations of heterogeneous individuals, who differ in different relevant aspects, is crucial, is the study of inequality dynamics and the mechanisms fostering respectively reducing inequality (see e.g. Dawid and Gemkow (2014), Dosi et al. (2013), Russo et al. (2016) for MABM analyses with such focus). Also, MABMs seem to be the natural tool to examine in a systematic way how the implications and effectiveness of certain policy measures depend on the level of inequality and the population distribution with respect to key economic characteristics. 


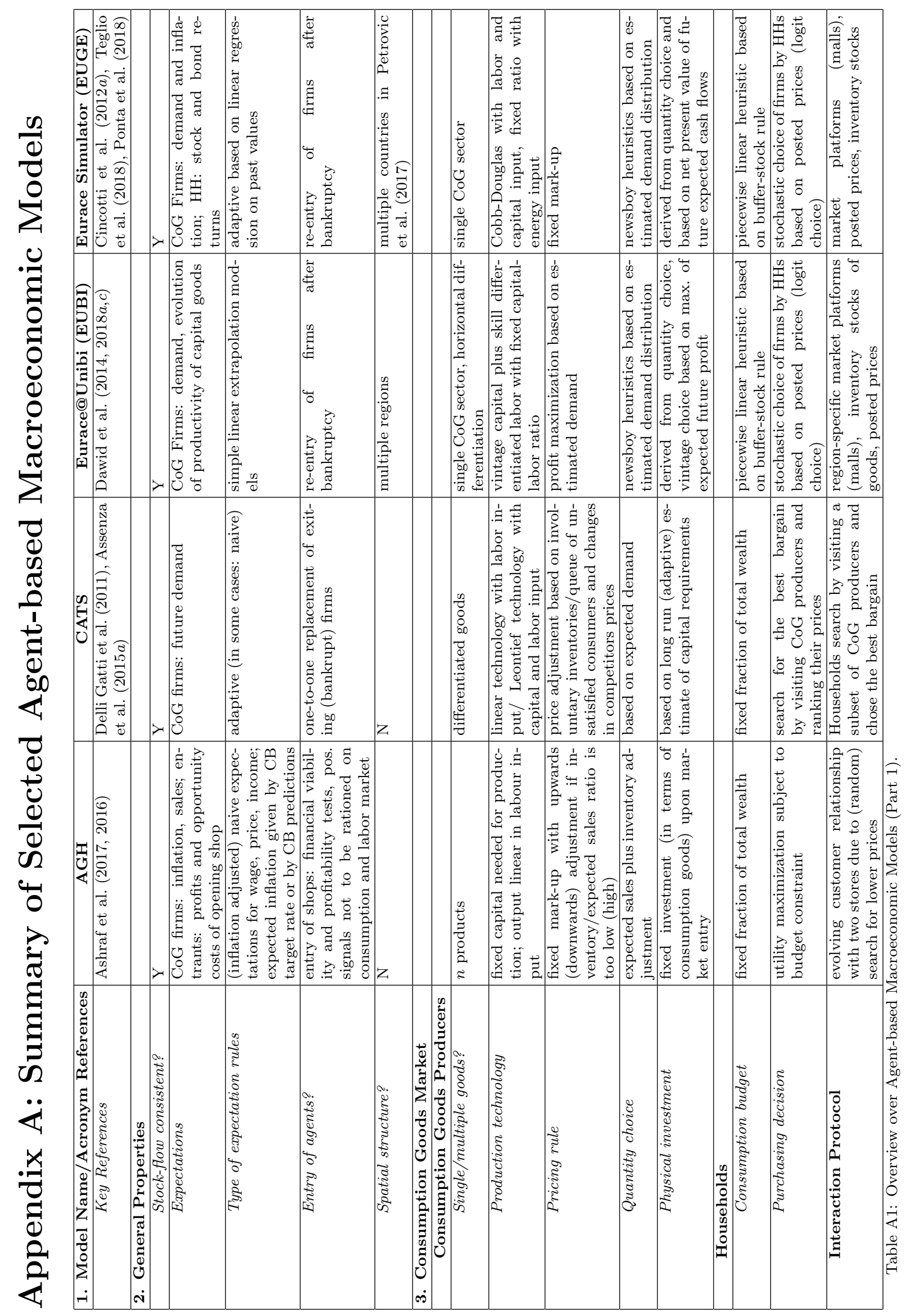




\begin{tabular}{|c|c|c|c|c|c|c|c|c|c|c|c|}
\hline 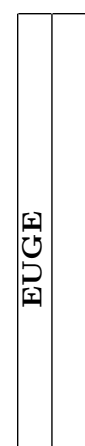 & 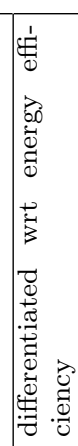 & 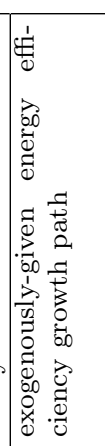 & 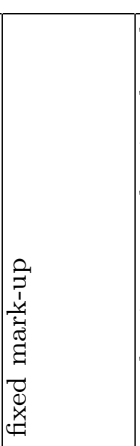 & 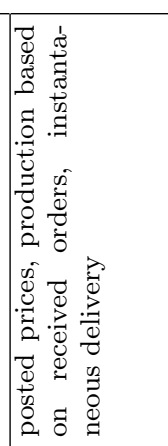 & & 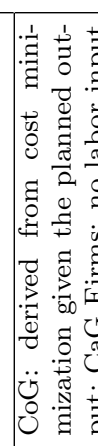 & 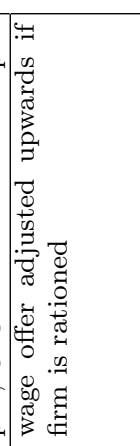 & 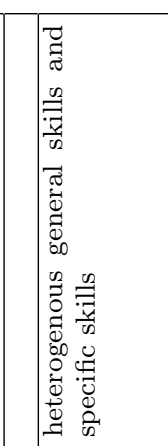 & 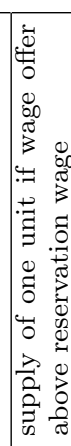 & 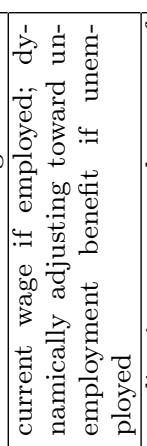 & 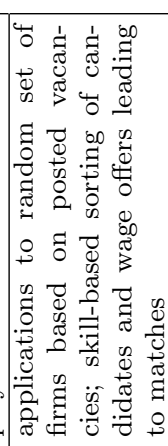 \\
\hline 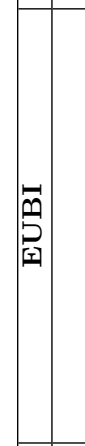 & 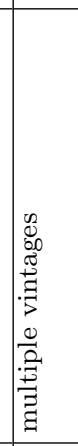 & 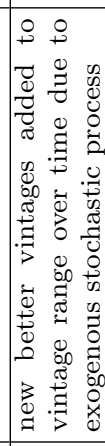 & 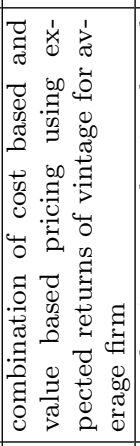 & 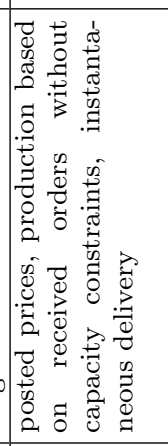 & & 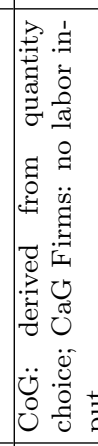 & 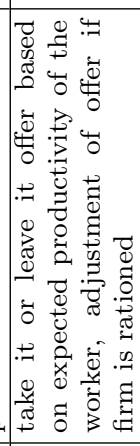 & 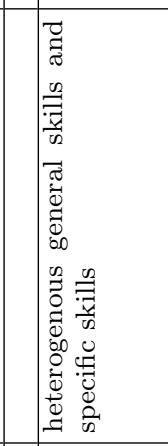 & 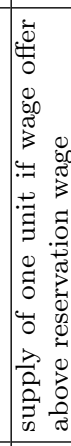 & 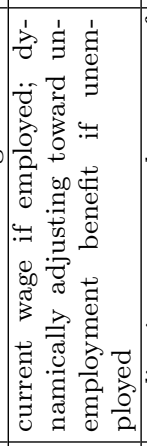 & 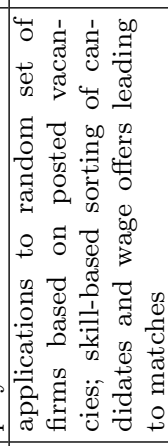 \\
\hline$\left|\begin{array}{l}n \\
0 \\
1 \\
0 \\
0\end{array}\right|$ & $\lambda$ & $\begin{array}{l}\Xi \\
0 \\
\Xi\end{array}$ & 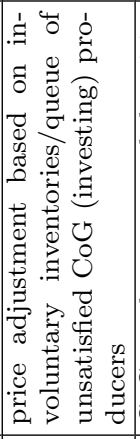 & 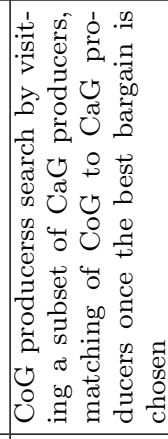 & & 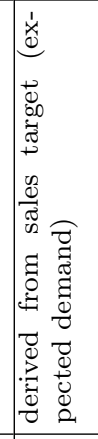 & 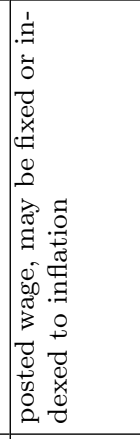 & 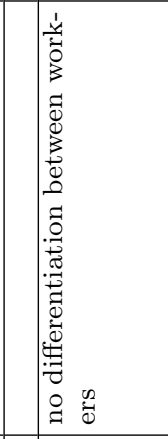 & 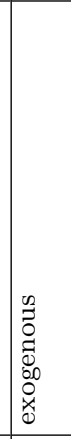 & . & 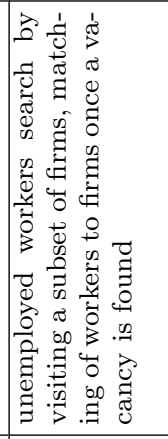 \\
\hline$\left|\begin{array}{l}\mathbb{T} \\
\mathbb{J} \\
\mathbb{4}\end{array}\right|$ & 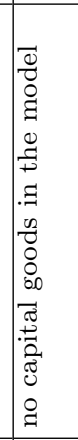 & 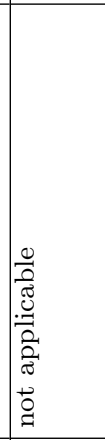 & 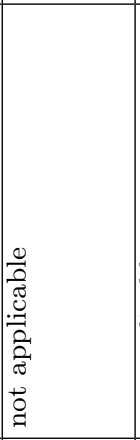 & 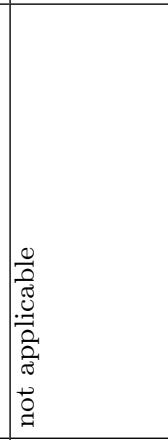 & & 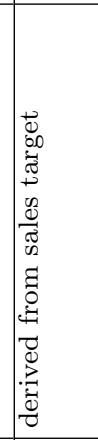 & 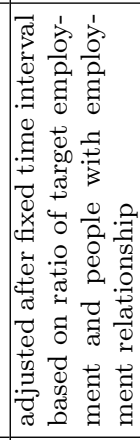 & 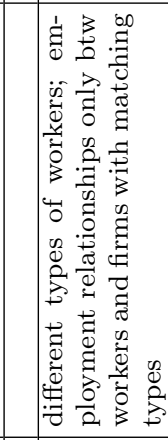 & 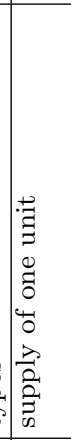 & 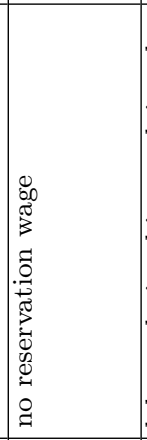 & 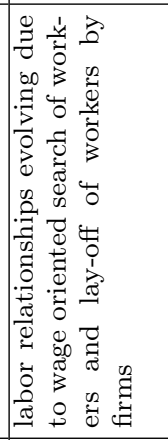 \\
\hline 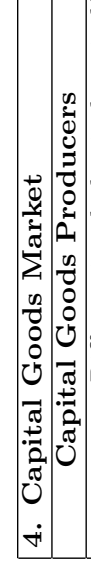 & 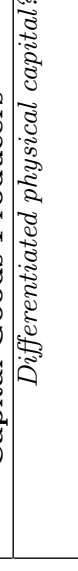 & 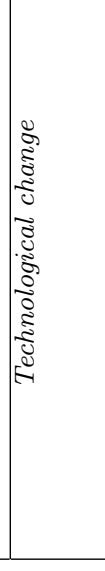 & 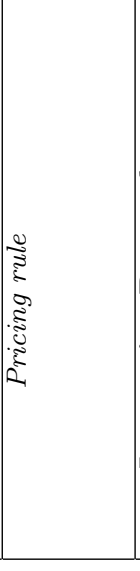 & 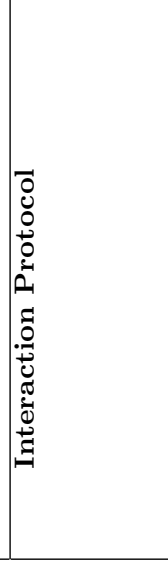 & 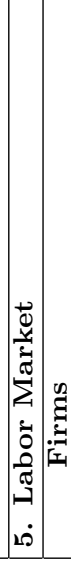 & 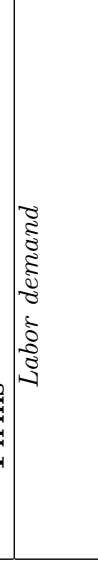 & 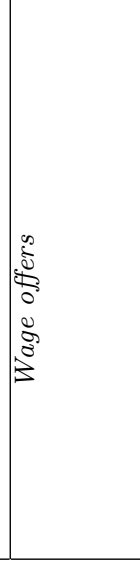 & 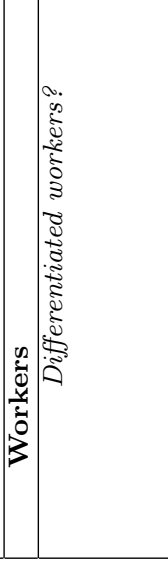 & 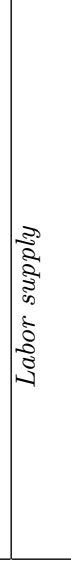 & 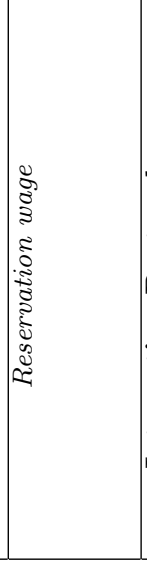 & 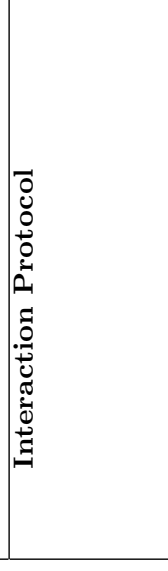 \\
\hline
\end{tabular}




\begin{tabular}{|c|c|c|c|c|c|c|c|c|c|c|c|c|c|c|}
\hline 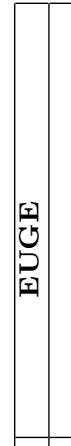 & 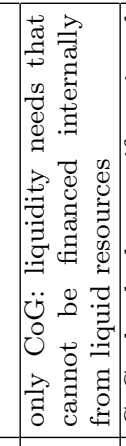 & 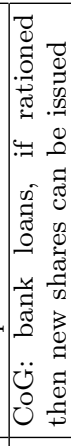 & 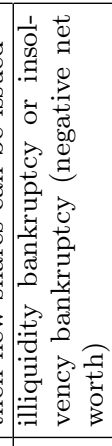 & & 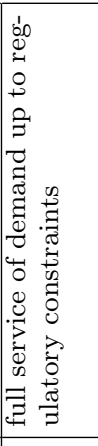 & 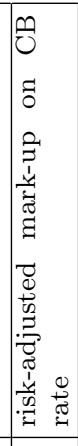 & 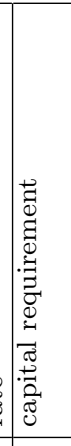 & 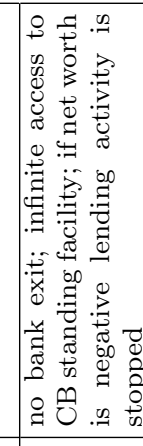 & 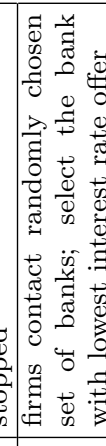 & & 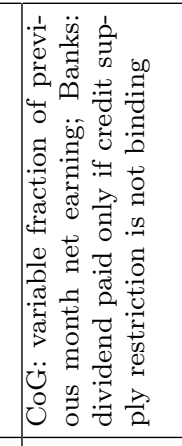 & & 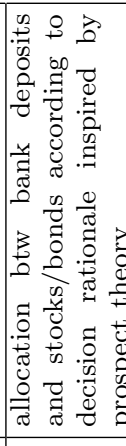 & 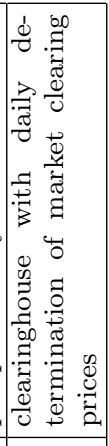 \\
\hline $\mid \begin{array}{l}0 \\
5 \\
n^{2}\end{array}$ & 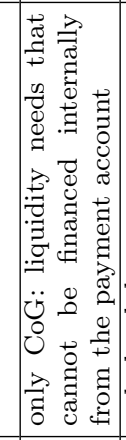 & 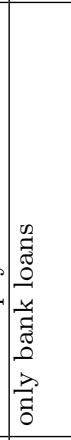 & 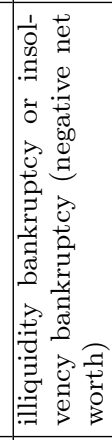 & & 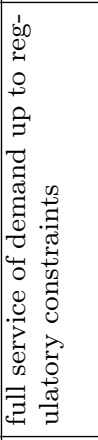 & 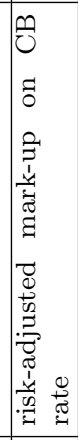 & 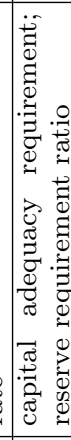 & 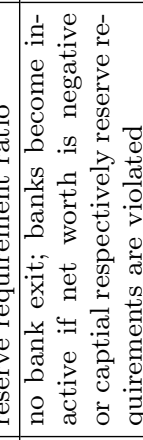 & 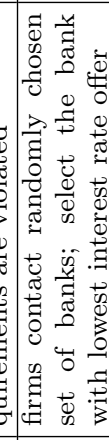 & & 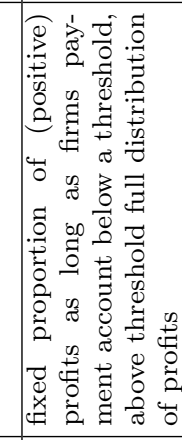 & & 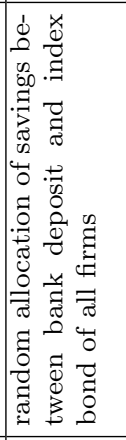 & 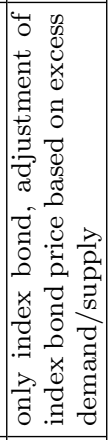 \\
\hline$\left|\begin{array}{l}n \\
\vdots \\
\vdots \\
0 \\
0\end{array}\right|$ & 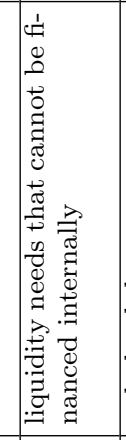 & 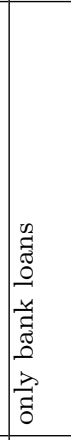 & 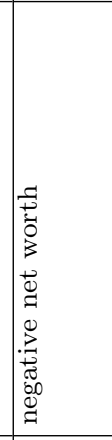 & & 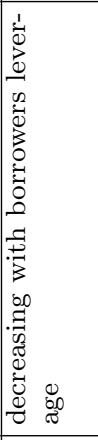 & 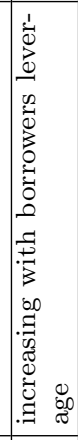 & 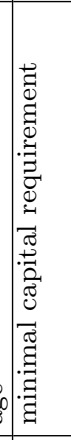 & 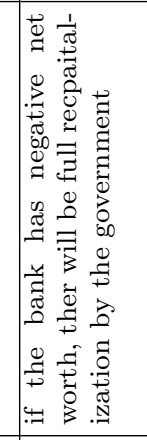 & 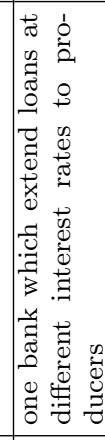 & & 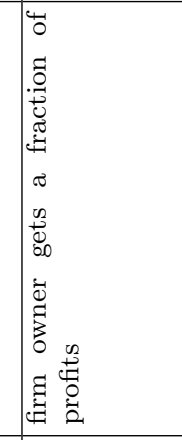 & & & 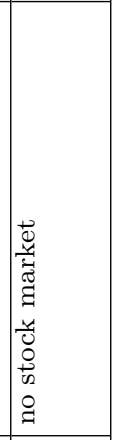 \\
\hline$\left|\begin{array}{l}\mathbb{Z} \\
\mathbb{Z} \\
\mathbb{Z}\end{array}\right|$ & 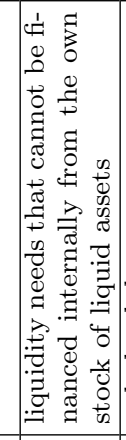 & 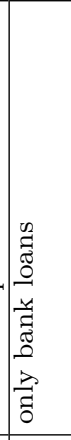 & 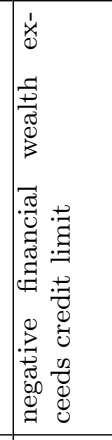 & & 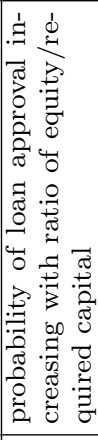 & 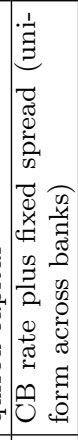 & 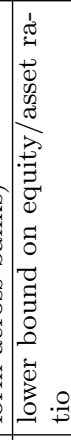 & 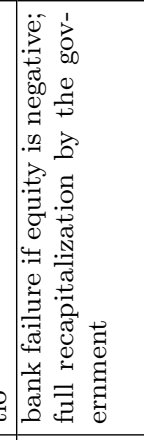 & 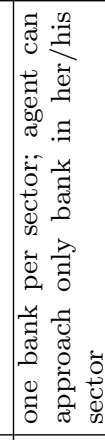 & & 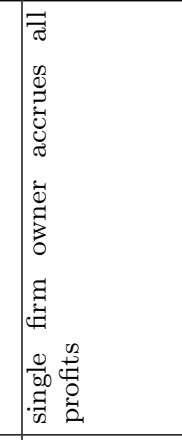 & & 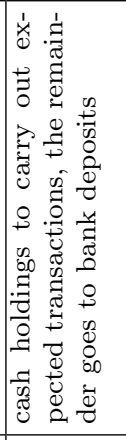 & 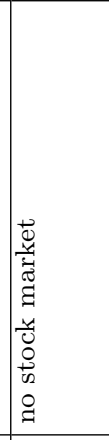 \\
\hline 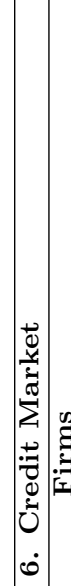 & 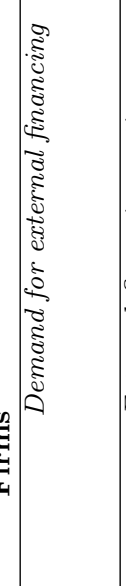 & 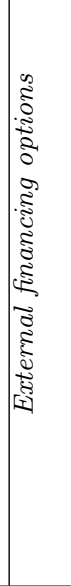 & 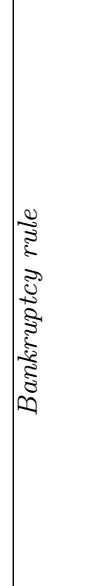 & $\pi$ & 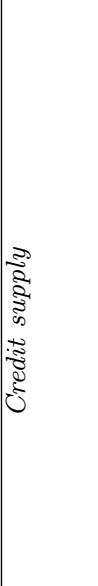 & 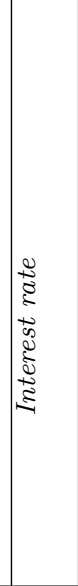 & 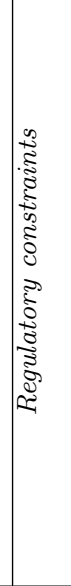 & 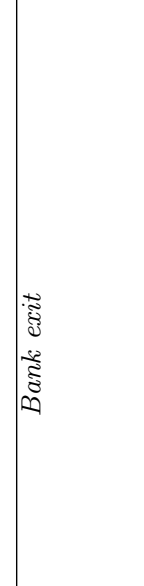 & 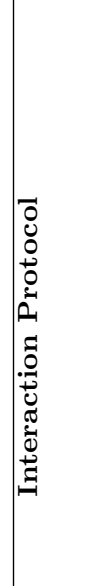 & 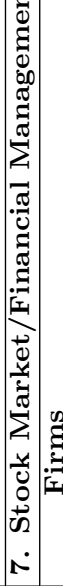 & 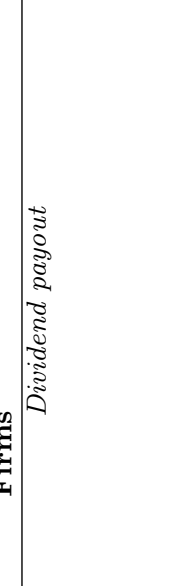 & & & 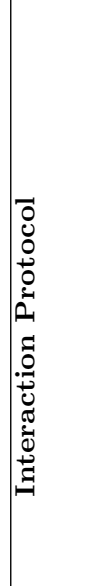 \\
\hline
\end{tabular}




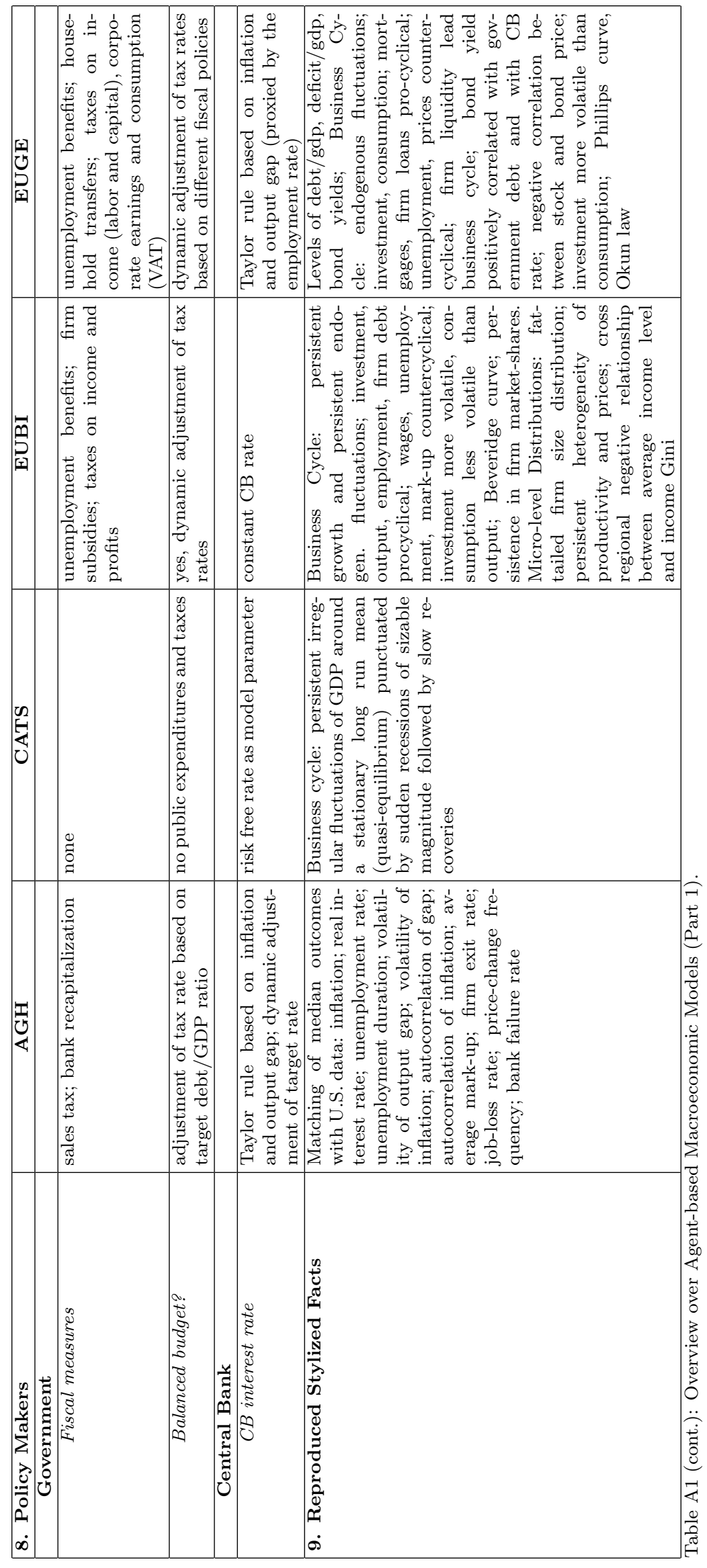




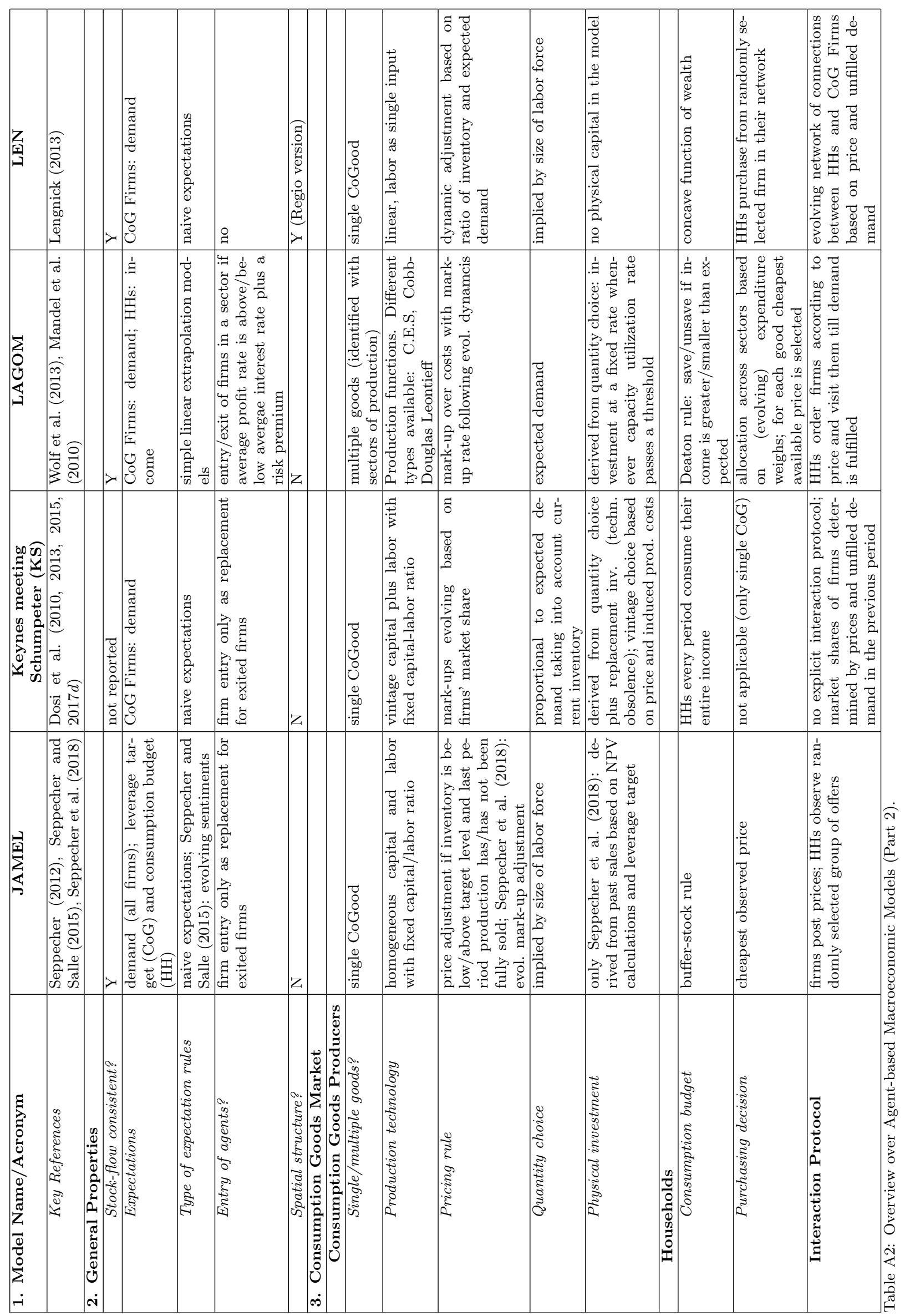




\begin{tabular}{|c|c|c|c|c|c|c|c|c|c|c|c|}
\hline 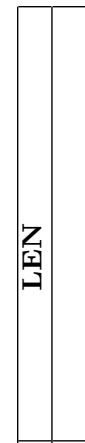 & 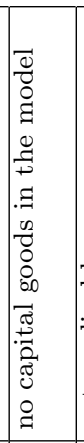 & 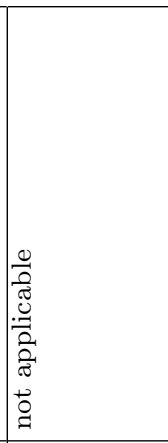 & 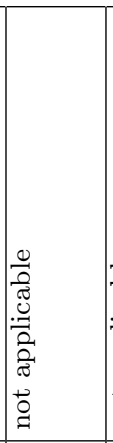 & 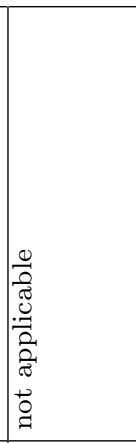 & & 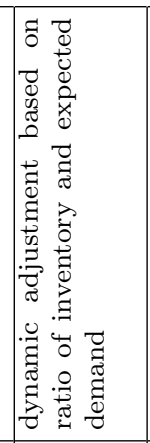 & 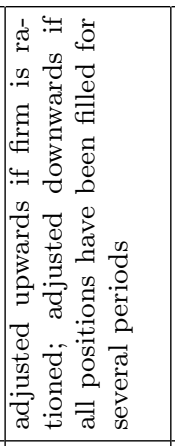 & 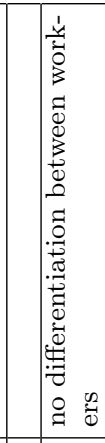 & 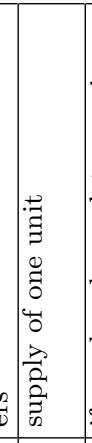 & 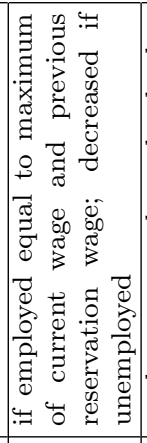 & 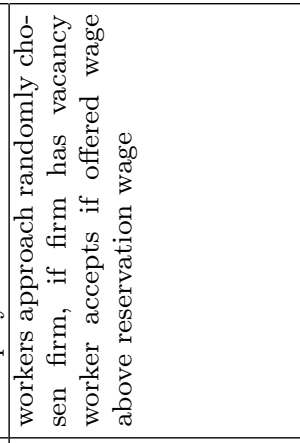 \\
\hline 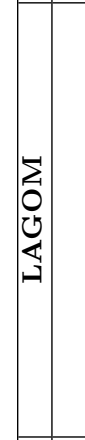 & 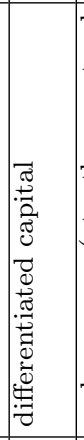 & 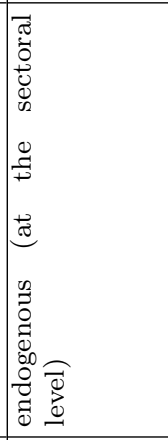 & 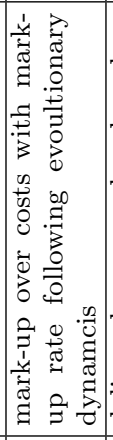 & 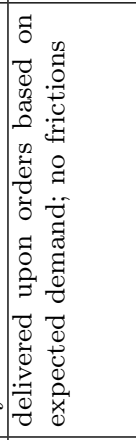 & & 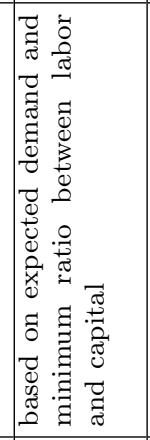 & 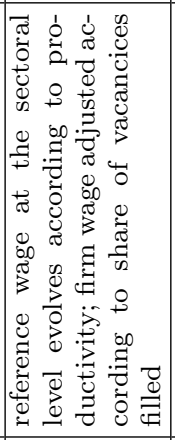 & 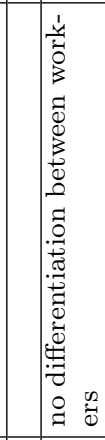 & 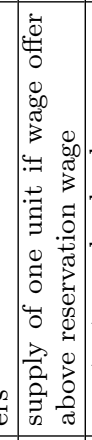 & 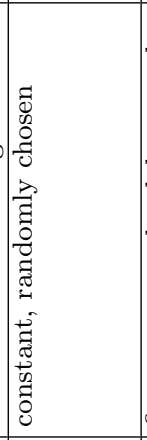 & 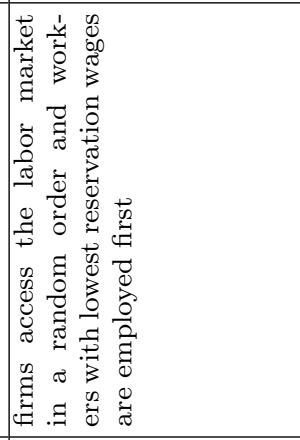 \\
\hline 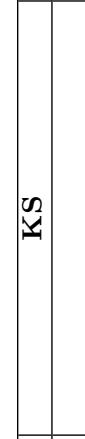 & 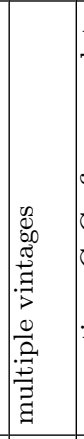 & 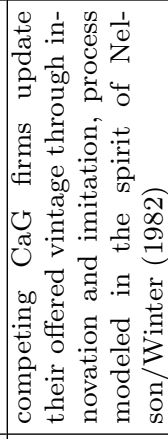 & 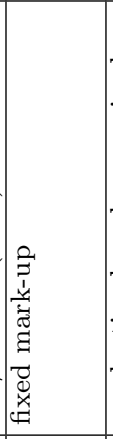 & 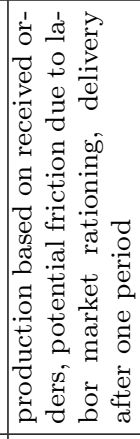 & & 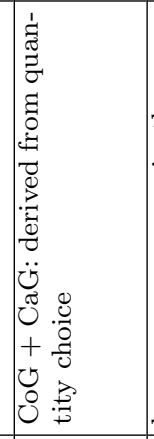 & 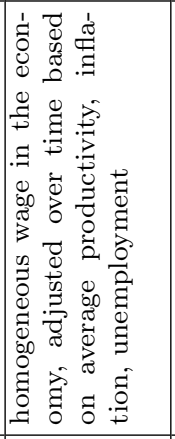 & 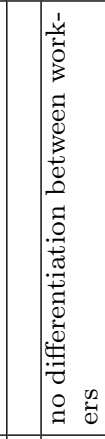 & 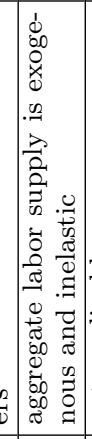 & 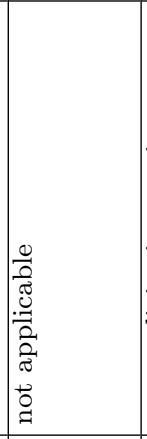 & 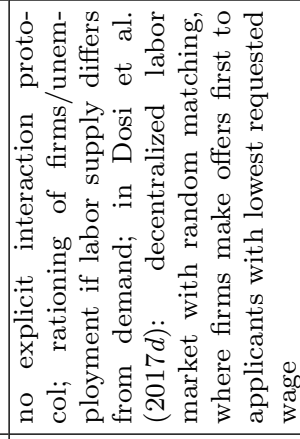 \\
\hline 氛 & 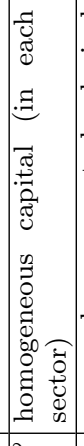 & 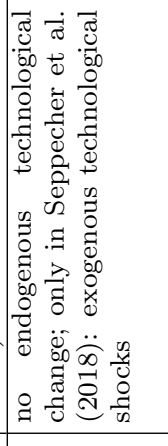 & 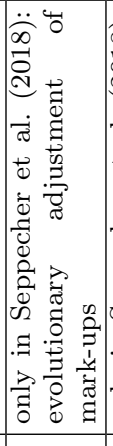 & 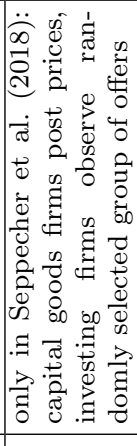 & & 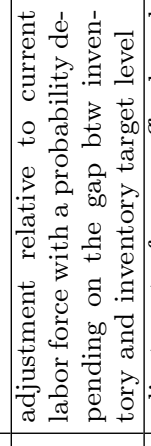 & 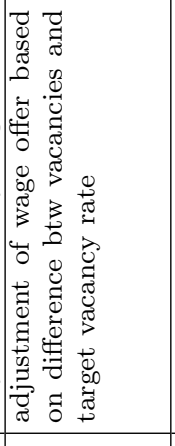 & 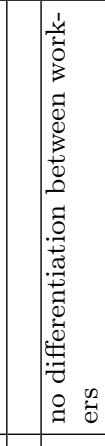 & 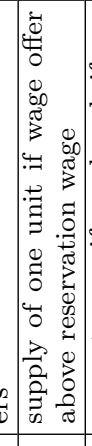 & 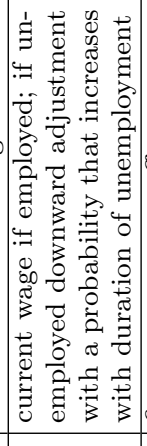 & 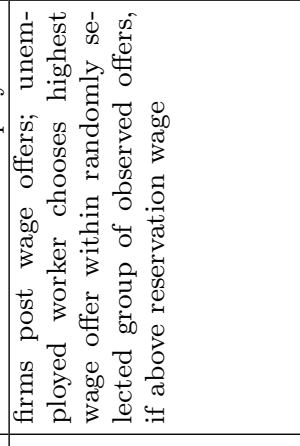 \\
\hline \begin{tabular}{|c|c|} 
& \\
& \\
& \\
& \\
0
\end{tabular} & 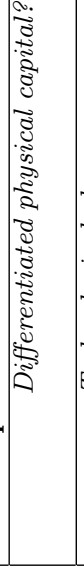 & 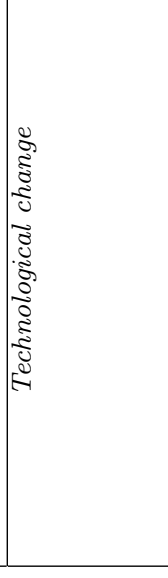 & 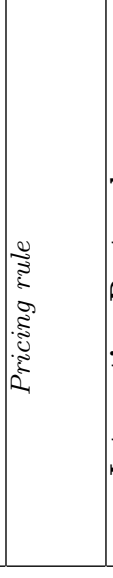 & 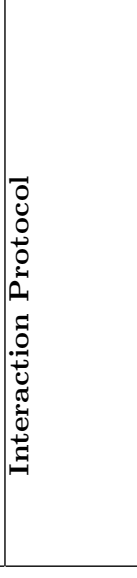 & & 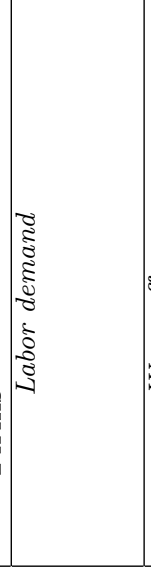 & 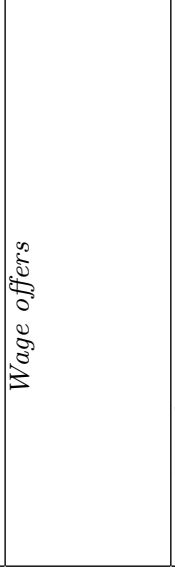 & 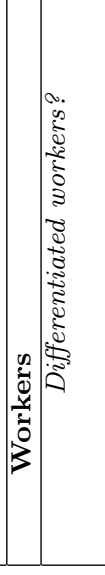 & 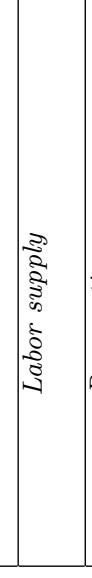 & 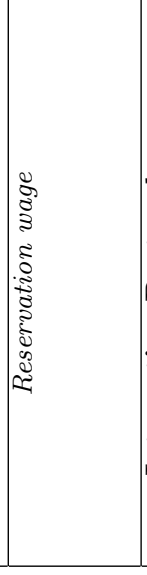 & 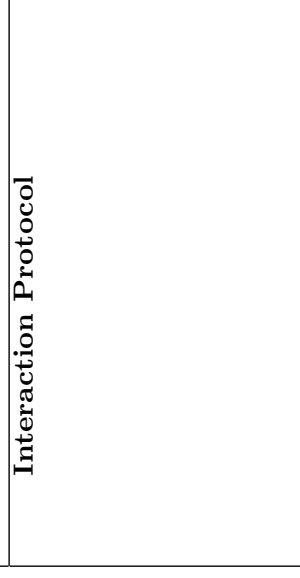 \\
\hline
\end{tabular}




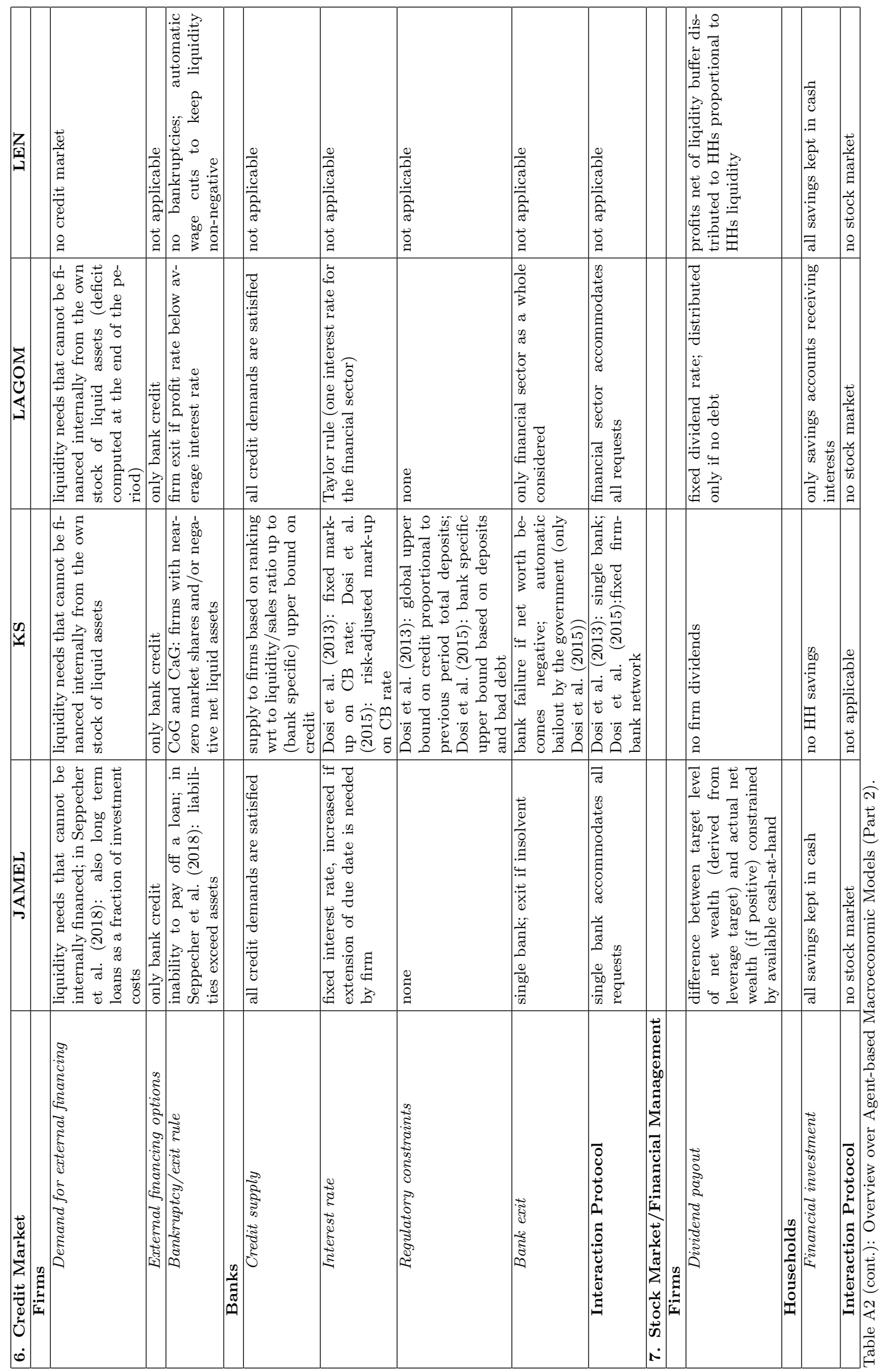




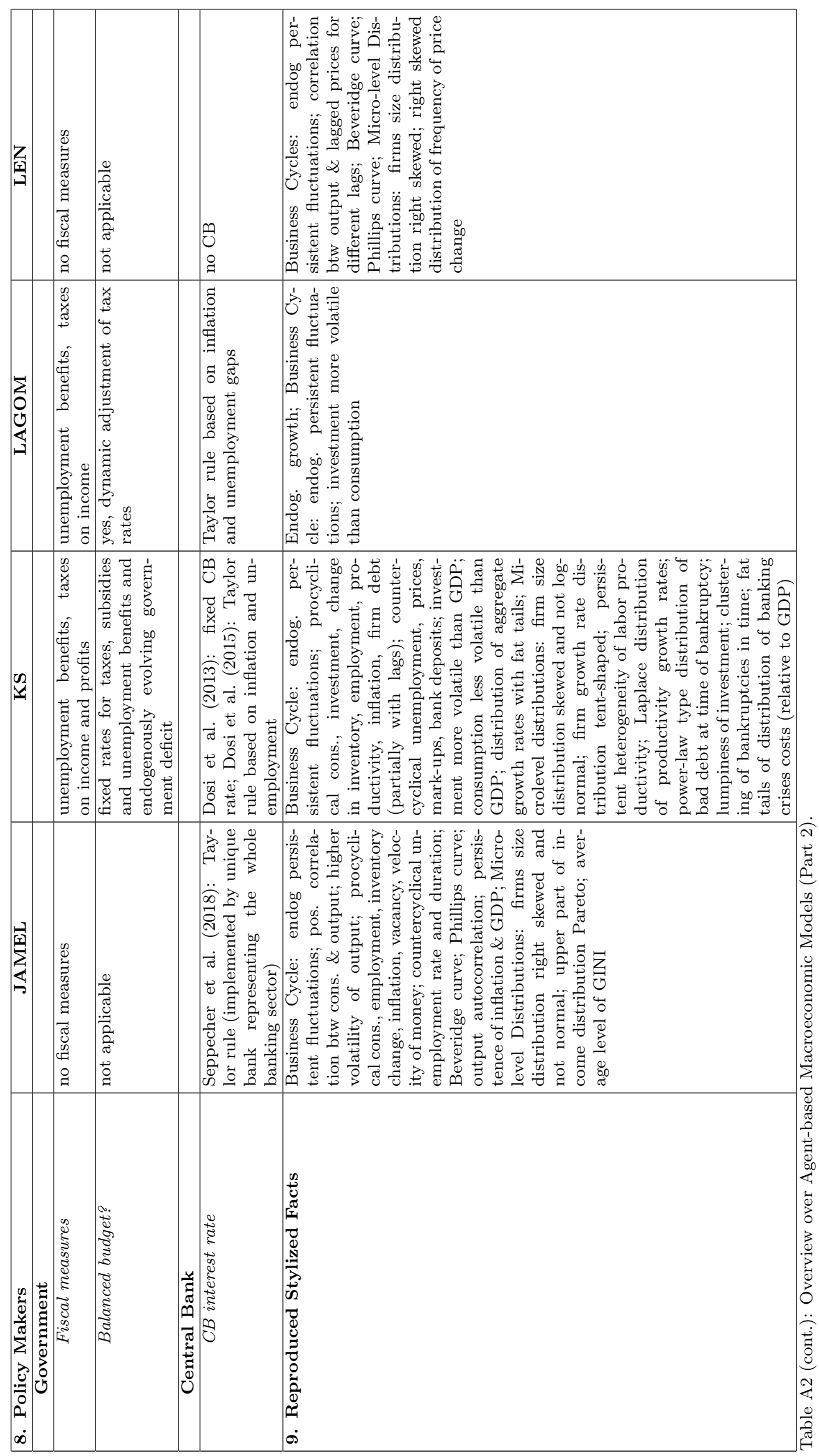




\section{Appendix B: List of symbols}

\begin{tabular}{|c|c|}
\hline Symbol & Meaning \\
\hline$C_{h}$ & total consumption of household $\mathrm{h}$ \\
\hline$Y_{h}$ & income of household h \\
\hline$S_{h}$ & saving of household h \\
\hline$C_{i, h}$ & consumption of variety $\mathrm{i}$ by household $\mathrm{h}$ \\
\hline$C$ & total households' consumption \\
\hline$C_{i}$ & households' demand for variety i \\
\hline$C_{i, t}^{e}$ & expected demand for variety i \\
\hline$Y_{i}$ & output of the i-th C-firm \\
\hline$Y_{k}$ & output of the k-th K-firm \\
\hline$J_{k}$ & demand for the $\mathrm{k}$-th $\mathrm{K}$-good \\
\hline$K_{i}$ & physical capital of firm i \\
\hline$I_{i}$ & investment of firm i \\
\hline$E_{i}$ & net worth or equity of firm i \\
\hline$\pi_{i}$ & profit of firm i \\
\hline$\omega_{i}$ & capacity utilization of firm i \\
\hline$N_{i}$ & employment at firm i \\
\hline$L_{i}$ & bank loans received by firm i \\
\hline$Y_{i}^{*}$ & desired or optimal output of firm i \\
\hline$Y$ & total output/income (gdp) \\
\hline$M C_{i}$ & marginal cost of firm i \\
\hline$A C_{i}$ & average cost of firm i \\
\hline$Q_{i}$ & sales of firm i \\
\hline$\Delta_{i}$ & inventories of firm i \\
\hline$\alpha$ & marginal productivity of labour \\
\hline$F_{c}$ & number of C-goods and of C-firms \\
\hline$F_{k}$ & number of K-goods and of K-firms \\
\hline$F$ & total number of firms \\
\hline$H$ & total number of households \\
\hline$Y_{h}^{p}$ & permament income of household $\mathrm{h}$ \\
\hline$Y_{h}^{a}$ & average income of household $\mathrm{h}$ \\
\hline $\bar{Y}_{h}^{a}$ & cut off value of the average income of household $h$ \\
\hline$R=1+r$ & gross nominal interest rate \\
\hline$W_{h}^{h}$ & human capital of household $\mathrm{h}$ \\
\hline$W_{h}^{f}$ & financial wealth of household $\mathrm{h}$ \\
\hline$M_{h}$ & money balances of household $h$ \\
\hline$w_{h}$ & reservation wage of household $\mathrm{h}$ \\
\hline$w_{i}$ & wage posted by firm i \\
\hline$w$ & wage \\
\hline$P_{i}$ & price of the i-th C-good \\
\hline$P_{k}$ & price of the k-th K-good \\
\hline$P$ & general price level \\
\hline
\end{tabular}




\begin{tabular}{|c|c|}
\hline$p_{i}$ & relative price of the i-th variety \\
\hline$y_{i}$ & output-to-loan ratio of firm i \\
\hline$\pi$ & inflation rate \\
\hline$k^{h}$ & capitalization factor (in AGH) \\
\hline$\xi$ & memory parameter in permanent income algorithm $(\mathrm{AGH})$ \\
\hline$\delta$ & desired inventory-to-sale ratio \\
\hline$\beta$ & exponent of capital in the production function \\
\hline$\omega^{f}$ & desired wealth-to-income ratio \\
\hline$\chi$ & parameter of quasi replicato dynamics of market share $(\mathrm{KS})$ \\
\hline$v$ & parameter of mark up dynamics (KS) \\
\hline$\zeta_{1}, \zeta_{2}$ & parameters of competitiveness equation $(\mathrm{KS})$ \\
\hline$\Omega_{i}$ & information set of firm i \\
\hline$e_{i}$ & forecasting error of firm i \\
\hline$\pi_{z}^{i}$ & fraction of sampled household that would good i at the price $P_{z}^{i}$ \\
\hline$\lambda_{i}$ & leverage of firm i \\
\hline$\lambda_{i}^{T}$ & target leverage of firm i \\
\hline$\mu_{i}$ & mark-up of firm i \\
\hline$\nu$ & velocity of adjustment of wealth to targeted wealth (EUBI) \\
\hline$\varepsilon$ & price elasticity of demand \\
\hline$\varepsilon^{i}$ & price elasticity of demand expected/estimated by firm i \\
\hline$C_{h}^{i}$ & total consumption expenditure estimated/expected by firm i \\
\hline$\eta$ & stochastic rate of change of the price set by each firm \\
\hline$\eta^{w}$ & stochastic rate of change of the wage posted by each firm \\
\hline$\eta^{h}$ & stochastic rate of change of the reservation wage \\
\hline$d_{h}^{u}$ & duration of the unemployment spell (JAMEL) \\
\hline $\bar{d}^{u}$ & cut off value of the unemployment spell (JAMEL) \\
\hline$\gamma$ & intensity of (price) competition among C-firms (EUBI) \\
\hline$\rho$ & intensity of adjustment of output to changes in invesntories (ADG) \\
\hline$c_{h}$ & propensity to consume out of human capital \\
\hline$c_{f}$ & propensity to consume out of financial wealth \\
\hline$c_{y}$ & propensity to consume out of income \\
\hline$f_{i}$ & market share of firm i (KS) \\
\hline$E_{i}$ & competitiveness of firm i (KS) \\
\hline $\bar{E}$ & average competitiveness of C-firms (KS) \\
\hline$s^{H}$ & propensity to save when optimist (JAMEL) \\
\hline$s^{L}$ & propensity to save when pessimist (JAMEL) \\
\hline$\pi^{m}$ & probability of adopting the consumer sentiment of the majority (JAMEL) \\
\hline$\pi^{s}$ & probability of searching for a job if unemployed (AGH) \\
\hline$\pi^{T}$ & inflation target of the central bank (AGH) \\
\hline$v$ & vintage of a machine tool \\
\hline$V$ & total number of vintages of a machine tool \\
\hline$Z_{c}$ & number of C-firms visited by a household to buy C-goods \\
\hline$Z_{k}$ & number of K-firms visited by a C-firm to buy a machine tool \\
\hline$Z_{e}$ & number of firms visited by a household searching for a job \\
\hline
\end{tabular}




\section{References}

Anufriev, M., Assenza, T., Hommes, C. \& Massaro, D. (2013), 'Interest rules and macroeconomic stability under hetergenous expectations', Macroeconomic Dynamics 17, 1574-1604.

Arifovic, J. (1995), 'Genetic algorithms and inflationary economies', Journal of Monetary Economics 36, 219-243.

Arifovic, J. (1996), 'The behavior of the exchange rate in the genetic algorithm and experimental economies', Journal of Political Economy 104, 510-541.

Arifovic, J., Bullard, J. \& Kostyshyna, O. (2013), 'Social learning and monetary policy rules', Economic Journal 123, 38-76.

Artinger, F. \& Gigerenzer, G. (2016), Heuristic pricing in an uncertain market: Ecological and constructivist rationality, Working Paper.

Ashraf, Q., Gershman, B. \& Howitt, P. (2016), 'How inflation affects macroeconomic performance: An agent-based computational investigation', Macroeconomic Dynamics 20, 558581.

Ashraf, Q., Gershman, B. \& Howitt, P. (2017), 'Banks, market organization, and macroeconomic performance: An agent-based computational analysis', Journal of Economic Behavior and Organization 135, 143-180.

Assenza, T., Delli Gatti, D. \& Grazzini, J. (2015a), 'Emergent dynamics of a macroeconomic agent based model with capital and credit', Journal of Economic Dynamics and Control 50, 5-28.

Assenza, T. \& Gatti, D. D. (2013), 'E pluribus unum: Macroeconomic modelling for multiagent economies', Journal of Economic Dynamics and Control 37, 1659-1682.

Assenza, T. \& Gatti, D. D. (2017), 'The financial transmissiom of shocks in a simple hybrid macroeconomic agent based model', forthcoming in Journal of Evolutionary Economics .

Assenza, T., Grazzini, J., Hommes, C. \& Massaro, D. (2015b), 'Pq strategies in monopolistic competition: Some insights from the lab', Journal of Economic Dynamics and Control 50, 62-77.

Balint, T., Lamperti, F., Mandel, A., Napoletano, M., Roventini, A. \& Sapio, A. (2017), 'Complexity and the economics of climate change: a survey and a look forward', Ecological Economics 158, 252-265.

Barde, S. (2017), 'A practical, accurate, information criterion for nth order markov processes', Computational Economics 50, 281-324.

Bargigli, L., Riccetti, L., Russo, A. \& Gallegati, M. (2014), 'Network analysis and calibration of the leveraged network-based financial accelerator', Journal of Economic Behavior and Organization 99(C), 109-125. 
Battiston, S., Delli Gatti, D., Gallegati, M., Greenwald, B. \& Stiglitz, J. (2007), 'Credit chains and bankruptcy propagation in production networks', Journal of Economic Dynamics and Control 31, 2061-2084.

Benes, J., Kumhof, M. \& Laxton, D. (2014), Financial crises in dsge models: A prototype model, IMF Working Paper 14/57, International Monetary Fund.

Bennett, R. \& Bergmann, B. (1986), A microsimulated model of the United States Economy, The John Hopkins University Press, Baltimore.

Bergman, B. (1974), 'A microsimulation of the macroeconomy with explicitly represented money flows', Annals of Economic and Social Measurement 3, 475-489.

Bernanke, B. \& Gertler, M. (1989), 'Agency costs, net worth, and business fluctuations', American Economic Review 79, 14-31.

Bernanke, B. \& Gertler, M. (1990), 'Financial fragility and economic performance', Quarterly Journal of Economics 105, 87-114.

Bernanke, B., Gertler, M. \& Gilchrist, S. (1999), The financial accelerator in a quantitative business cycle framework, in J. Taylor \& M. Woodford, eds, 'Handbook of Macroeconomics, Volume 1', Amsterdam: North-Holland, pp. 1341-1393.

Breitmoser, Y. (2016), 'The axiomatic foundation of logit', MPRA Paper No. 74334.

Caiani, A., Godin, A., Caverzasi, E., Gallegati, M., Kinsella, S. \& Stiglitz, J. E. (2016a), 'Agent based-stock flow consistent macroeconomics: Towards a benchmark model', Journal of Economic Dynamics and Control 69, 375-408.

Caiani, A., Russo, A. \& Gallegati, M. (2016b), Does inequality hamper innovation and growth? An AB-SFC analysis, Working paper available at ssrn: http://papers.ssrn.com/sol3/papers.cfm?abstract_id=2790911.

Carroll, C. (1992), How does future income affect current consumption?, Working paper 126, board of governors of the federal reserve system.

Carroll, C. (1997), 'Buffer-stock saving and the life cycle/permanent income hypothesis', Quarterly Journal of Economics 112(1), 1-55.

Carroll, C. (2003), 'Macroeconomic expectations of households and professional forecasters', Quarterly Journal of Economics 118, 269-298.

Carroll, C. (2009), 'Precautionary saving and the marginal propensity to consume out of permanent income', Journal of Monetary Economics 56(6), 780-790.

Carroll, C. \& Summers, L. (1991), Consumption growth parallels income growth: Some new evidence, in B. Bernheim \& J. Shoven, eds, 'National Saving and Economic Performance', Chicago: University of Chicago Press, pp. 305-348.

Catullo, E., Gallegati, M. \& Palestrini, A. (2015), 'Towards a credit network based early warning indicator for crises', Journal of Economic Dynamics and Control 50, 78-97. 
Chiarella, C. \& Di Guilmi, C. (2017), 'Monetary policy and debt deflation: Some computational experiments', Macroeconomic Dynamics 21, 214-242.

Cincotti, S., Raberto, M. \& Teglio, A. (2010), 'Credit money and macroeconomic instability in the agent-based model and simulator eurace', Economics: The Open-Access, OpenAssessment E-Journal 4, 1-32.

Cincotti, S., Raberto, M. \& Teglio, A. (2012a), The eurace macroeconomic model and simulator, in M. Aoki, K. Binmore, S. Deakin \& H. Gintis, eds, 'Complexity and Institutions: Markets, Norms and Corporations', New York: Palgrave Macmillan, pp. 81-106.

Cincotti, S., Raberto, M. \& Teglio, A. (2012b), 'Macroprudential policies in an agent-based artificial economy', Revue de l'OFCE 124, 205-234.

Cioppa, T. \& Lucas, T. (2007), 'Efficient nearly orthogonal and space-filling latin hypercubes', Technometrics pp. 45-55.

Colander, D., Howitt, P., Kirman, A., Leijonhufvud, A. \& Mehrling, P. (2008), 'Beyond dsge models: Toward an empirically based macroeconomics', The American Economic Review: Papers and Proceedings 98, 236-240.

Dawid, H. \& Gemkow, S. (2014), 'How do social networks contribute to wage inequality? insights from an agent-based analysis', Industrial and Corporate Change 23, 1171-1200.

Dawid, H., Gemkow, S., Harting, P. \& Neugart, M. (2009), 'On the effects of skill upgrading in the presence of spatial labor market frictions: an agent-based analysis of spatial policy design', Journal of Artifical Societies and Social Simulation - JASSS 12, 4.

Dawid, H., Gemkow, S., Harting, P. \& Neugart, M. (2012), 'Labor market integration policies and the convergence of regions: the role of skills and technology diffusion', Journal of Evolutionary Economics 22, 543-562.

Dawid, H., Gemkow, S., Harting, P., Neugart, M., Kabus, K. \& Wersching, K. (2008), 'Skills, innovation and growth: an agent-based policy analysis', Journal of Economics and Statistics 228, 251-275.

Dawid, H., Gemkow, S., Harting, P., van der Hoog, S. \& Neugart, M. (2018a), Agent-based macroeconomic modeling and policy analysis: The eurace@unibi model, in S.-H. Chen \& M. Kaboudan, eds, 'Handbook on Computational Economics and Finance', Oxford University Press.

Dawid, H. \& Harting, P. (2012), Capturing firm behavior in agent-based models of industry evolution and macroeconomic dynamics, in G. Bünsdorf, ed., 'Evolution, Organization and Economic Behavior', Edward Elgar, chapter 6.

Dawid, H., Harting, P. \& Neugart, M. (2013), 'Cohesion policy and inequality dynamics: Insights from a heterogeneous agents macroeconomic model', Bielefeld Working Papers in Economics and Management No. 26/2013.

Dawid, H., Harting, P. \& Neugart, M. (2014), 'Economic convergence: Policy implications from a heterogeneous agent model', Journal of Economic Dynamics and Control 44, 54-80. 
Dawid, H., Harting, P. \& Neugart, M. (2018b), 'Fiscal transfers and regional economic growth', forthcoming in Review of International Economics .

Dawid, H., Harting, P., van der Hoog, S. \& Neugart, M. (2018c), 'A heterogeneous agent macroeconomic model for policy evaluation: Improving transparency and reproducibility', forthcoming in Journal of Evolutionary Economics .

Deaton, A. (1991), 'Saving and liquidity constraints', Econometrica 59, 1221-1248.

Deissenberg, C., van der Hoog, S. \& Dawid, H. (2008), 'Eurace: A massively parallel agentbased model of the european economy', Applied Mathematics and Computation 204, 541552.

Delli Gatti, D. \& Desiderio, S. (2015), 'Monetary policy experiments in an agent-based model with financial frictions', Journal of Economic Interaction and Coordination 10, 265-286.

Delli Gatti, D., Di Guilmi, C., Gaffeo, E., Giulioni, G., Gallegati, M. \& Palestrini, A. (2005), 'A new approach to business fluctuations: heterogeneous interacting agents, scaling laws and financial fragility', Journal of Economic Behavior and Organization 56, 489-512.

Delli Gatti, D., Gallegati, M., Cirillo, P., Desiderio, S. \& Gaffeo, E. (2011), Macroeconomics from the Bottom-up, Springer-Verlag, Berlin.

Delli Gatti, D., Gallegati, M., Greenwald, B., Russo, A. \& Stiglitz, J. (2010), 'The financial accelerator in an evolving credit network', Journal of Economic Dynamics and Control 34, 1627-1650.

Delli Gatti, D., Gallegati, M., Greenwald, B., Russo, A. \& Stiglitz, J. E. (2006), 'Business fluctuations in a credit-network economy', Physica A: Statistical Mechanics and its Applications 370(1), 68-74.

Delli Gatti, D., Gallegati, M., Greenwald, B., Russo, A. \& Stiglitz, J. E. (2009), 'Business fluctuations and bankruptcy avalanches in an evolving network economy', Journal of Economic Interaction and Coordination 4(2), 195-212.

Dosi, G., Fagiolo, G., Napoletano, M. \& Roventini, A. (2013), 'Income distribution, credit and fiscal policies in an agent-based Keynesian model', Journal of Economic Dynamics and Control 37, 1598-1625.

Dosi, G., Fagiolo, G., Napoletano, M., Roventini, A. \& Treibich, T. (2015), 'Fiscal and monetary policies in complex evolving economies', Journal of Economic Dynamics and Control 52, 166-189.

Dosi, G., Fagiolo, G. \& Roventini, A. (2006), 'An evolutionary model of endogenous business cycles', Computational Economics 27, 3-34.

Dosi, G., Fagiolo, G. \& Roventini, A. (2008), 'The microfoundations of business cycles: an evolutionary multi-agent model', Journal of Evolutionary Economics 18, 413-432.

Dosi, G., Fagiolo, G. \& Roventini, A. (2010), 'Schumpeter meeting Keynes: a policy-friendly model of endogenous growth and business cycles', Journal of Economic Dynamics and Control 34, 1748-1767. 
Dosi, G., Napoletano, M., Roventini, A. \& Treibich, T. (2017a), 'Micro and macro policies in the Keynes plus Schumpeter evolutionary models', Journal of Evolutionary Economics 27, 63-90.

Dosi, G., Pereira, M., Roventini, A. \& Virgillito, M. (2018), The effects of labour market reforms upon unemployment and income inequalities: an agent based model approach, forthcoming in Socio-Economic Review.

Dosi, G., Pereira, M., Roventini, A. \& Virgillito, M. (2017b), Causes and consequences of hysteresis: Aggregate demand, productivity and employment, LEM Working Paper No. $2017 / 07$.

Dosi, G., Roventini, A. \& Russo, E. (2017c), Endogenous growth and global divergence in a multi-country agent-based model, LEM Working Paper No. 2017/32.

Dosi, G., Pereira, M., Roventini, A. \& Virgillito, M. (2017d), 'When more flexibility yields more fragility: the microfoundations of Keynesian aggregate unemployment', Journal of Economic Dynamics and Control 81, 162-186.

Eliason, G. (1977), 'Competition and market processes in a simulation model of the swedish economy', American Economic Review 67, 277-281.

Eliason, G. (1984), 'Micro-heterogeneity of firms and the stability of industrial growth', Journal of Economic Behavior and Organization 5, 249-274.

Erlingsson, E. J., Raberto, M., Stefnsson, H. \& Sturluson, J. T. (2013), Integrating the housing market into an agent-based economic model, in A. Teglio, S. Alfarano, E. CamachoCuena \& M. Gins-Vilar, eds, 'Managing market complexity', Lecture Notes in Economics and Mathematical Systems, Springer.

Erlingsson, E., Teglio, A., Cincotti, S., Stefansson, H., Sturluson, J. T. \& Raberto, M. (2014), 'Housing market bubbles and business cycles in an agent-based credit economy', ECONOMICS: THE OPEN-ACCESS, OPEN-ASSESSMENT E-JOURNAL 8.

Fagiolo, G. \& Roventini, A. (2011), 'On the scientific status of economic policy: A tale of alternative paradigms', forthcoming in the Knowledge Engineering Review .

Fagiolo, G. \& Roventini, A. (2017), 'Macroeconomic policy in dsge and agent-based models redux: New developments and challenges ahead', Journal of Artificial Societies and Social Simulation 20, 1.

Fontana, M. (2010), 'The santa fe perspective on economics: Emerging patterns in the science of complexity', History of Economic Ideas 18, 167-196.

Gaffeo, E., Catalano, M., Clementi, F., Delli Gatti, D., Gallegati, M. \& Russo, A. (2007), 'Reflections on modern macroeconomics: Can we travel along a safer road?', Physica A: Statistical Mechanics and its Applications 382(1), 68-74.

Gaffeo, E., Gallegati, M. \& Gostoli, U. (2015), "An agent-based "proof of principle" for walrasian macroeconomic theory', Computational and Mathematical Organization Theory 21), 150-183. 
Gali, J. (2008), Monetary policy,inflation and the business cycle, Princeton University Press, Princeton, New Jersey.

Gigerenzer, G. \& Gaissmaier, W. (2011), 'Heuristic decision making', Annual Review of Psychology 62, 451-482.

Goudet, O., Kant, J.-D. \& Ballot, G. (2015), Forbidding fixed duration contracts: Unfolding the opposing effects with a multiagent model of the french labour market, in F. Amblard, F. Miguel, A. Blanchet \& B. Gaudou, eds, 'Advances in Artificial Economics, Lecture Notes in Economics and Mathematical Systems, Vol. 676', Springer: Berlin, pp. 151-167.

Grazzini, J., M.G.Richiardi \& Tsionas, M. (2017), 'Bayesian estimation of agent-based models', Journal of Economic Dynamics and Control 77, 26-47.

Greenwald, B. \& Stiglitz, J. (1993), 'Financial markets imperfections and business cycles', Quarterly Journal of Economics 108, 77-113.

Gualdi, S., Tarzia, M., Zamponi, F. \& Bouchaud, J. (2015), 'Tipping points in macroeconomic agent-based models', Journal of Economic Dynamics and Control 50(1), 29-61.

Guerini, M. \& Moneta, A. (2017), 'A method for agent-based models validation', Journal of Economic Dynamics and Control 82, 125-141.

Haas, A. \& Jaeger, C. (2005), 'Agents, bayes, and climate risks - a modular modelling approach', Advances in Geosciences 4, 3-7.

Haber, G. (2008), 'Monetary and fiscal policy analysis with an agent-based macroeconomic model', Journal of Economics and Statistics 228, 276-205.

Haldane, A. (2016), The dappled world, http://www.bankofengland.co.uk/publications/pages/ speeches/2016/937.aspx.

Harting, P. (2015), 'Stabilization policies and long term growth: Policy implications from an agent-based macroeconomic model', Bielefeld Working Papers in Economics and Management No. 06-2015.

Haruvy, E., Roth, A. \& Unver, U. (2006), 'The dynamics of law clerk matching: An experimental and computational investigation of proposals for reform of the market', Journal of Economic Dynamics and Control 30, 457-486.

Holcombe, M., Coakley, S., Kiran, M., Chin, S., Greenough, C., Worth, D., Cincotti, S., Raberto, M., Teglio, A., Deissenberg, C., van der Hoog, S., Dawid, H., Gemkow, S., Harting, P. \& Neugart, M. (2013), 'Large-scale modeling of economic systems', Complex Systems 22, 175-191.

Hommes, C. (2013), Behavioral Rationality and Heterogeneous Expectations in Complex Economic Systems, Cambridge University Press.

Hommes, C., Sonnemans, J., Tuinstra, J. \& van de Velden, H. (2005), 'Coordination of expectations in asset pricing experiments', Review of Financial Studies 18, 955-980. 
Howitt, P. (2012), 'What have central bankers learned from modern macroeconomic theory?', Journal of Macroeconomics 34, 11-22.

Kirman, A. (1992), 'Whom or what does the representative individual represent?', Journal of Economic Perspectives 6, 117-136.

Kirman, A. (2016), 'Ants and nonoptimal self-organization: Lessons for macroeconomics', Macroeconomic Dynamics 20, 601-621.

Kiyotaki, N. \& Moore, J. (1997), 'Credit cycles', Journal of Political Economy 105, 211-248.

Klimek, P., Poledna, S., Farmer, J. \& Thurner, S. (2015), 'To bail-out or to bail-in? answers from an agent-based model', Journal of Economic Dynamics and Control 50, 144-154.

Krug, S. (2015), The interaction between monetary and macroprudential policy: Should central banks "lean against the wind" to foster macrofinancial stability?, Economics Working Paper, Christian-Albrechts-Universitt Kiel, Department of Economics, No. 2015-08.

Krug, S. \& Wohltmann, H.-W. (2016), 'Shadow banking, financial regulation and animal spirits: An ace approach', Economics Working Paper, Christian-Albrechts-Universität Kiel, Department of Economics, No. 2016-08.

Lamperti, F., Dosi, G., Napoletano, M., Roventini, A. \& Sapio, A. (2017), Faraway, so close: Coupled climate and economic dynamics in an agent-based integrated assessment model, LEM Working Paper 2017/12.

Lengnick, M. (2013), 'Agent-based macroeconomics: A baseline model', Journal of Economic Behavior and Organization 86, 102-120.

Lengnick, M. \& Wohltmann, H.-W. (2011), Agent-based financial markets and new keynesian macroeconomics: A synthesis, Economics working paper, christian-albrechts-universität kiel, department of economics, no. 2011,09.

Lengnick, M. \& Wohltmann, H.-W. (2016), 'Optimal monetary policy in a new keynesian model with animal spirits and financial markets', Journal of Economic Dynamics and Control 64, 148-165.

Mandel, A., Jaeger, C., Fuerst, S., Lass, W., Lincke, D., Meissner, F., Pablo-Marti, F. \& Wolf, S. (2010), 'Agent-based dynamics in disaggregated growth models', Centre dEconomie de la Sorbonne Working Paper 10077.

Minsky, H. (1963), Can "it" happen again, in D. Carson, ed., 'Banking and Monetary Studies', Richard D. Irwin: Homewood.

Napoletano, M., Dosi, G., Fagiolo, G. \& Roventini, A. (2012), 'Wage formation, investment behavior and growth regimes: an agent-based analysis', Revue de l'OFCE 124, 235-261.

Napoletano, M., Roventini, A. \& Gaffard, J.-L. (2015), Time-varying fiscal multipliers in an agent-based model with credit rationing, GREDEG Working Paper No. 2015-30. 
Neugart, M. \& Richiardi, M. (2018), Agent-based models of the labor market, in S.-H. Chen \& M. Kaboudan, eds, 'Handbook on Computational Economics and Finance', Oxford University Press.

Neveu, A. (2013), 'Fiscal policy and business cycle characteristics in a heterogeneous agent macro model', Journal of Economic Behavior and Organization 90, 224-240.

Ozel, B., Nathanael, R., Raberto, M., Teglio, A. \& Cincotti, S. (2016), Macroeconomic implications of mortgage loans requirements: An agent based approach, Working Papers 2016/05, Economics Department, Universitat Jaume I, Castelln.

Petrovic, M., Ozel, B., Teglio, A., Raberto, M. \& Cincotti, S. (2017), Eurace open: An agent-based multi-country model, Universtiy Jaume I Working Papers No. 2017/09.

Piva, M. \& Vivarelli, M. (2009), 'Corporate skills as an ex-ante incentive to R\&D investment', International Journal of Manpower 30, 835-852.

Ponta, L., Raberto, M. \& Cincotti, S. (2018), 'An agent-based stock-flow consistent model of the sustainable transition in the energy sector', Ecological Economics 145, 274-300.

Popoyan, L., Napoletano, M. \& Roventini, A. (2017), 'Taming macroeconomic instability: Monetary and macro-prudential policy interactions in an agent-based model', Journal of Economic Behavior and Organization 134, 117-140.

Raberto, M., Cincotti, S. \& Teglio, A. (2014), Fiscal consolidation and sovereign debt risk in balance-sheet recessions: an agent-based approach, in P. T. L. Manica, ed., 'Economic Policy and the Financial Crisis', Routledge.

Raberto, M., Nathanael, R., Ozel, B., Teglio, A. \& Cincotti, S. (2017), Credit-driven business cycles in an agent-base macro model, in M. S.-W. H. Hanappi, S.s Katsikides, ed., 'Theory and method of evolutionary political economy', Routledge.

Raberto, M., Ozel, B., Ponta, L., Teglio, A. \& Cincotti, S. (2018), 'From financial instability to green finance: the role of banking and monetary policies in the eurace model', forthcoming in Journal of Evolutionary Economics .

Raberto, M., Teglio, A. \& Cincotti, S. (2008), 'Integrating real and financial markets in an agent-based economic model: an application to monetary policy design', Computational Economics 32, 147-162.

Raberto, M., Teglio, A. \& Cincotti, S. (2012), 'Debt, deleveraging and business cycles: an agent-based perspective', Economics, The Open-Access, Open-Assessment E-Journal 6(2012-27).

Riccetti, L., Russo, A. \& Gallegati, M. (2013), 'Leveraged network-based financial accelerator', Journal of Economic Dynamics and Control 37(8), 1626-1640.

Riccetti, L., Russo, A. \& Gallegati, M. (2015), 'An agent based decentralized matching macroeconomic model', Journal of Economic Interaction and Coordination vol. 10(2), 305-332. 
Riccetti, L., Russo, A. \& Gallegati, M. (2016a), 'Financialisation and crisis in an agent based macroeconomic model', Economic Modelling 52(PA), 162-172.

Riccetti, L., Russo, A. \& Gallegati, M. (2016b), 'Stock market dynamics, leveraged networkbased financial accelerator and monetary policy', International Review of Economics and Finance 43(C), 509-524.

Riccetti, L., Russo, A. \& Gallegati, M. (2017), 'Financial regulation and endogenous macroeconomic crises', forthcoming in Macroeconomic Dynamics .

Riccetti, L., Russo, A. \& Gallegati, M. (2018), 'Financial regulation and endogenous macroeconomic crises', forthcoming in Macroeconomic Dynamics .

Richiardi, M. (2017), 'The future of agent-based modeling', Eastern Economic Journal 43, 271-287.

Romer, P. (2016), The trouble with macroeconomics, forthcoming in The American Economist.

Russo, A., Catalano, M., Gaffeo, E., Gallegati, M. \& Napoletano, M. (2007), 'Industrial dynamics, fiscal policy and r\&d: Evidence from a computational experiment', Journal of Economic Behavior and Organization 64, 426-447.

Russo, A., Riccetti, L. \& Gallegati (2016), 'Increasing inequality, consumer credit and financial fragility in an agent based macroeconomic model', Journal of Evolutionary Economics 26, 25-47.

Salle, I. (2015), 'Modeling expectations in agent-basedmodelsan application to central bank's communication and monetary policy', Economic Modelling 46, 130-141.

Salle, I., Yldzoglu, M. \& Senegas, M.-A. (2013), 'Inflation targeting in a learning economy: An abm perspective', Economic Modelling 34, 114-128.

Seppecher, P. (2012), 'Flexibility of wages and macroeconomic instability in an agent-based compuational model with endogenous money', Macroeconomic Dynamics 16, 284-297.

Seppecher, P. \& Salle, I. (2015), 'Deleveraging crises and deep recessions: a behavioural approach', Applied Economics 47, 3771-3790.

Seppecher, P., Salle, I. \& Lavoie, M. (2018), 'What drives markups? evolutionary pricing in an agent-based stock-flow consistent macroeconomic model', forthcoming in Industrial and Corporate Change.

Simon, H. (1959), 'Theories of decision-making in economics and behavioral science', American Economic Review 49, 253-283.

Sims, C. (1980), 'Macroeconomics and reality', Econometrica 48, 1-48.

Sinitskaya, E. \& Tesfatsion, L. (2015), 'Macroeconomies asconstructivelyrationalgames', Journal of Economic Dynamics and Control 61, 152-182. 
Teglio, A., Cincotti, S., Erlingsson, E., Raberto, M., Stefansson, H. \& Sturluson, J. (2014), Subprime lending and financial inequality in an agent-based model, in S. Leitner \& F. Wall, eds, 'Artificial Economics and Self Organization', Lecture Notes in Economics and Mathematical Systems, Springer.

Teglio, A., Mazzocchetti, A., Ponta, L., Raberto, M. \& Cincotti, S. (2018), 'Budgetary rigour with stimulus in lean times: Policy advices from an agent-based model', forthcoming in Journal of Economic Behavior and Organization .

Teglio, A., Raberto, M. \& Cincotti, S. (2010), Endogenous credit dynamics as source of business cycles in the eurace model, in M. L. Calzi, L. Milone \& P. Pellizzari, eds, 'Progress in Artificial Economics. Computational and Agent-Based Models', Lecture Notes in Economics and Mathematical Systems, Springer.

Teglio, A., Raberto, M. \& Cincotti, S. (2012), 'The impact of banks capital adequacy regulation on the economic system: an agent-based approach', Advances in Complex Systems 15.

Tesfatsion, L. (2006), Agent-based computational economics: A constructive approach to economic theory, in L. Tesfatsion \& K. Judd, eds, 'Handbook of Computational Economics, Volume II', North-Holland, pp. 831-880.

Tesfatsion, L. \& Judd, K. E. (2006), Handbook of Computational Economics II: Agent-Based Computational Economics, North-Holland.

Trichet, J. (2010), Reflections on the nature of monetary policy non-standard measures and finance theory, http://www.ecb.europa.eu/press/key/date/2010/html/sp101118.en.html.

van der Hoog, S. (2018), 'The limits to credit growth: Mitigation policies and macroprudential regulations to foster macrofinancial stability and sustainable debt', forthcoming in Computational Economics .

van der Hoog, S. \& Dawid, H. (2017), 'Bubbles, crashes and the financial cycle: Insights from a stock-flow consistent agent-based macroeconomic model', forthcoming in Macroeconomic Dynamics .

Wolf, S., Fürst, S., Mandel, A., Lass, W., Lincke, D., Pablo-Martí, F. \& Jaeger, C. (2013), 'A multi-agent model of several economic regions', Environmental Modelling \& Software 44, 25-43. 